\title{
Limits of three-dimensional gravity and metric kinematical Lie algebras in any dimension
}

\author{
Javier Matulich, ${ }^{a}$ Stefan Prohazka $^{a}$ and Jakob Salzer ${ }^{b}$ \\ ${ }^{a}$ Université Libre de Bruxelles and International Solvay Institutes, \\ Physique Mathématique des Interactions Fondamentales, \\ Campus Plaine - CP 231, Bruxelles B-1050, Belgium \\ ${ }^{b}$ Departament de Física Quàntica i Astrofísica, Institut de Ciències del Cosmos, \\ Universitat de Barcelona, Martíi Franquès 1, E-08028 Barcelona, Spain \\ E-mail: javier.matulich@ulb.ac.be, stefan.prohazka@ulb.ac.be, \\ jakob.salzer@icc.ub.edu
}

ABSTRACT: We extend a recent classification of three-dimensional spatially isotropic homogeneous spacetimes to Chern-Simons theories as three-dimensional gravity theories on these spacetimes. By this we find gravitational theories for all carrollian, galilean, and aristotelian counterparts of the lorentzian theories. In order to define a nondegenerate bilinear form for each of the theories, we introduce (not necessarily central) extensions of the original kinematical algebras. Using the structure of so-called double extensions, this can be done systematically. For homogeneous spaces that arise as a limit of (anti-)de Sitter spacetime, we show that it is possible to take the limit on the level of the action, after an appropriate extension. We extend our systematic construction of nondegenerate bilinear forms also to all higher-dimensional kinematical algebras.

Keywords: Chern-Simons Theories, Classical Theories of Gravity, Space-Time Symmetries

ArXiv ePrint: 1903.09165

Christa Zauner gewidmet. 


\section{Contents}

1 Introduction 1

2 Chern-Simons theory and homogeneous spacetimes 4

2.1 Three-dimensional gravity as a Chern-Simons theory 4

$\begin{array}{lll}2.2 & \text { Chern-Simons theories for three-dimensional homogeneous spacetimes } & 7\end{array}$

3 Kinematical limits of three-dimensional gravity $\quad 9$

3.1 Kinematical limits 9

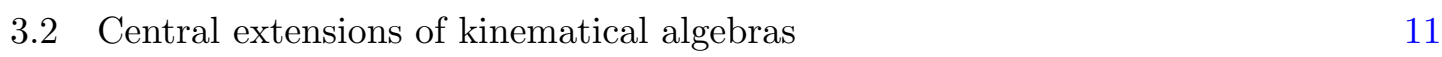

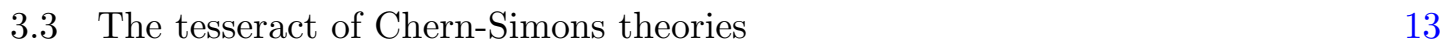

$\begin{array}{ll}3.4 \text { The most general action } & 15\end{array}$

4 Double extensions $\quad 16$

$\begin{array}{lll}4.1 & \text { Definition of double extensions } & 17\end{array}$

$\begin{array}{lll}4.2 & \text { (A)dS, Poincaré, and carrollian algebras } & 19\end{array}$

$\begin{array}{lll}4.3 & \text { Double extended (A)dS-Galilei and Galilei } & 19\end{array}$

5 Non-contracting theories $\quad 21$

5.1 Torsional galilean theories 21

$\begin{array}{ll}5.2 & \text { Carrollian light cone } \\ 5.3 & 22\end{array}$

$\begin{array}{lll}5.3 & \text { Aristotelian } & 23\end{array}$

5.3.1 Aristotelian static and torsional static 24

$\begin{array}{lll}\text { 5.3.2 } & \mathbb{R} \times \mathbb{H}^{2} \text { and } \mathbb{R} \times \mathbb{S}^{2} & 25\end{array}$

$\begin{array}{lll}5.3 .3 & \text { A24 } 25\end{array}$

6 Generalization to higher dimensions $\quad 25$

6.1 (Anti-)de Sitter algebras 26

6.2 Metric Poincaré algebra and the Maxwell algebra 26

$\begin{array}{lll}6.3 & \text { Metric carrollian algebras } & 27\end{array}$

$\begin{array}{ll}\text { 6.4 Metric galilean algebras } & 28\end{array}$

$\begin{array}{ll}\text { 6.5 Metric para-galilean and static algebra } & 29\end{array}$

$\begin{array}{lll}6.6 & \text { Non-contracting algebras } & 30\end{array}$

$\begin{array}{lll}\text { 6.6.1 Torsional galilean algebras } & 30\end{array}$

$\begin{array}{ll}\text { 6.6.2 Carrollian light cone algebra } & 31\end{array}$

$\begin{array}{lll}\text { 6.6.3 Aristotelian algebras } & 31\end{array}$

$\begin{array}{lll}7 & \text { The coadjoint kinematical algebras and their limits } & 32\end{array}$

$\begin{array}{lll}8 & \text { Summary of results } & 33\end{array}$ 
9 Discussion $\quad 36$

9.1 Three-dimensional kinematical theories 36

$\begin{array}{lll}9.2 & \text { Higher dimensions } & 37\end{array}$

$\begin{array}{ll}\text { A Conventions } & 38\end{array}$

A.1 Conventions for $2+1$ dimensions $\quad 39$

$\begin{array}{lll}\text { A.2 } & \text { Most general centrally extended (A)dS } & 39\end{array}$

$\begin{array}{lll}\text { A.3 Riemannian spacetimes } & 40\end{array}$

B Kinematical algebras, homogeneous spaces, and Cartan geometries $\quad 40$

C The full action and equations of motion 43

\section{Introduction}

The crucial rôle played by symmetries in three-dimensional gravity becomes obvious from the intriguing possibility to write gravity in a gauge theory formulation. The Chern-Simons (CS) action based on either of the gauge algebras $\mathfrak{i s o}(2,1), \mathfrak{s o}(3,1), \mathfrak{s o}(2,2)$, is classically equivalent to the Einstein-Hilbert action in its first-order formulation with zero, positive or negative cosmological constant, respectively $[1,2]$. Since three-dimensional gravity does not allow for propagating degrees of freedom, the solutions of the respective theories are consequently locally three-dimensional Minkowski, de Sitter (dS), or anti-de Sitter (AdS), with the gauge algebras corresponding to the symmetries of the respective spacetime. Gravity on $\mathrm{AdS}_{3}$ in particular has received a great amount of attention as one finds that, imposing the right boundary conditions [3], the asymptotic symmetries of $\mathrm{AdS}_{3}$ yield the infinitedimensional symmetries of a two-dimensional conformal field theory (CFT). This set-up presents one of the best studied instances of the AdS/CFT duality [4-6]. The intimate relation of CS theories to Wess-Zumino-Witten (WZW) models $[7,8]$ is a reason the gauge formulation of $\mathrm{AdS}_{3}$ gravity appears to be particularly suited for a better understanding of the correspondence [9].

The above mentioned symmetry algebras, $\mathfrak{i s o}(2,1), \mathfrak{s o}(3,1), \mathfrak{s o}(2,2)$, are examples of kinematical Lie algebras, that are associated to three-dimensional homogeneous spacetimes on which they act transitively; for the above mentioned these spacetimes are precisely three-dimensional Minkowski, $\mathrm{dS}_{3}$, and $\mathrm{AdS}_{3}$, respectively. ${ }^{1}$ From the action on the homogeneous spacetime the generators of a kinematical algebra inherit an interpretation of rotations, boosts, spatial and temporal translations. In fact, any homogeneous spacetime is completely specified by a kinematical algebra $\mathfrak{k}$ and a choice of subalgebra $\mathfrak{b}$ that singles out the transformations associated to boosts and rotations. For instance, for all three symmetry algebras mentioned above, the subalgebra $\mathfrak{b}$ is the three-dimensional Lorentz algebra $\mathfrak{s o}(2,1)$. The pair $(\mathfrak{k}, \mathfrak{b})$ determining the homogeneous space is called Klein pair. Assuming $\mathcal{K}$ and $\mathcal{B}$ are the simply connected Lie groups with Lie algebra $\mathfrak{k}$ and $\mathfrak{b}$, respectively, the homogeneous spacetime is then given by the quotient $\mathcal{K} / \mathcal{B}$.

\footnotetext{
${ }^{1}$ For precise definitions and further details see appendix B.
} 
In this work we want to define CS theories for all possible three-dimensional spatially isotropic homogeneous spacetimes. These homogeneous spacetimes can be regarded as generalization of maximally symmetric spacetimes dropping the assumption of the existence of a nondegenerate tangent space metric. The resulting CS theories are consequently gravitational theories that may not allow for a description in terms of a metric, i.e., they are non- or ultrarelativistic theories of gravity that have instead of the metric a galilean or carrollian structure on the tangent space.

The interest in gravitational theories based on galilean symmetries has been rekindled recently due to their relevance for holographic correspondences [10-17] and effective field theories [18-21] for condensed matter systems. Ultrarelativistic gravity theories on the other hand could help to elucidate the structure of field theories with carrollian symmetries, which appear at event horizons [22, 23] and at null infinity (and arguably also at spatial infinity $[24-26]) .^{2}$ Given the simplicity of gravity in three dimensions, it suggests itself as an interesting testing ground for the study of gravitational theories with non-lorentzian symmetries; see [30-32] for CS theories of the Carroll group and [33-37] for works on the (AdS-)Galilei group and supersymmetric extensions thereof.

A CS theory for a homogeneous spacetime is defined by two ingredients

- a Klein pair $(\mathfrak{k}, \mathfrak{b})$ with $\mathfrak{k}$ a kinematical Lie algebra and $\mathfrak{b}$ a subalgebra determining boosts and rotations;

- a symmetric, invariant, nondegenerate bilinear form, i.e., an invariant metric on $\mathfrak{k}$.

In order to write down a CS theory for all three-dimensional homogeneous spacetimes one has to, firstly, classify all Klein pairs giving rise to a three-dimensional spacetime and, secondly, specify an invariant metric for all of them.

A first classification of kinematical Lie algebras and their associated homogeneous spacetimes was provided in a seminal paper by Bacry and Lévy-Leblond [38] (see also [39]) where they showed how these algebras can be obtained by successive Inönü-Wigner (IW) contractions starting from the symmetry algebra $\mathfrak{s o}(D+1,1)[\mathfrak{s o}(D, 2)]$ of $(D+1)$ dimensional [anti-]de Sitter space. ${ }^{3}$ In this way one recovers the well-known Poincaré algebra iso $(D, 1)$, the nonrelativistic galilean algebras, and the ultrarelativistic carrollian algebras. In summary, they find a cube of kinematical algebras connected by limiting procedures. One of the main results of the present work is a lift of the cube of kinematical algebras of Bacry-Lévy-Leblond to a cube of three-dimensional gravity theories (cf. figure 1) connected by various limits.

More recently, the classification of homogeneous spacetimes was generalized to include spacetimes that do not arise as limits from (anti-)de Sitter space as well as aristotelian spacetimes, which lack boost invariance [40]. We include these non-contracting spacetimes in our discussion of all possible three-dimensional CS theories.

\footnotetext{
${ }^{2}$ More precisely: the structure at null infinity is only a conformal carrollian structure. This structure is left invariant by the infinite-dimensional Bondi-van der Burg-Metzner-Sachs (BMS) group [27-29].

${ }^{3}$ The authors of [38] restricted to $3+1$ dimensions. It was only recently shown that there is no change for higher dimensions [40] (a property that is not true if solely the kinematical Lie algebras are considered [41]).
} 
Let us now turn to the second ingredient in the definition of a CS theory. The existence of an invariant metric is guaranteed for [semi] simple Lie algebras such as the [A]dS algebra $\mathfrak{s o}(3,1)[\mathfrak{s o}(2,2)]$. However, apart from the (A)dS algebra none of the other kinematical algebras appearing in the cube of Bacry-Lévy-Leblond are semisimple. In line with this, one finds that the galilean algebras do not allow for an invariant metric. Yet the threedimensional Poincaré algebra iso $(2,1)$ while not being semisimple allows for an invariant metric, in contrast to all higher dimensions; a fact that was crucial in the construction of [2].

This raises the question which Lie algebras (or, for our purpose, kinematical Lie algebras) allow for an invariant metric. The answer is provided by a theorem of Medina and Revoy [42, 43] stating that all such Lie algebras are either abelian, simple, or socalled double extensions (or direct products of these ingredients). We will show that the Poincaré algebra allows for an invariant metric precisely because it can be understood as a double extension.

Given the fact that the galilean algebras do not allow for an invariant metric it appears that our program to define a CS theory for all three-dimensional homogeneous spacetimes is already doomed to fail. However, the theorem of Medina and Revoy also provides us with the tools to circumvent this conclusion. We find that the double extension structure can be used as a bootstrap to construct out of any kinematical algebra without invariant metric a new extended algebra that has one by construction. The new algebra and the original kinematical algebra share the same homogeneous spacetime. However, this extension might not be unique, and one of the central goals of this work is to find the one with minimal additional generators.

With this tool at our disposal, together with the classification of all three-dimensional kinematical spacetimes [40], we arrive at our main result which is a classification of all Chern-Simons theories based on the gauging of three-dimensional kinematical Klein pairs.

As mentioned above, we will also show that all CS actions based on the kinematical algebras of the cube of Bacry-Lévy-Leblond can be obtained as limits of the trivially twice centrally extended (A)dS algebra. These central extensions become nontrivial after the IW contraction and lead to kinematical algebras having the form of double extensions with invariant metric. ${ }^{4}$ A similar approach for the case of the galilean algebras was pioneered in $[33,34]$ and has recently been revived $[35,36]$. Remarkably these limits are not only a limit of the equations of motion, but also generalize to the action principle, which might be crucial for going beyond the classical level.

Having discussed three-dimensional homogeneous spacetimes, we will turn to higherdimensional kinematical algebras. We will not discuss gauge theories based on these algebras. Nevertheless, we find that the construction of metric Lie algebras out of these higher-dimensional kinematical algebras, using the double extension bootstrap, will lead to interesting Lie algebras, some of which appear to be novel while others are well-known in the literature. We finally provide a version of the cube of Bacry-Lévy-Leblond for metric Lie algebras in any dimension. This means that we find a particular double extension of the (A)dS algebra that allows to take a well-defined limit, both on the level of the algebra

\footnotetext{
${ }^{4}$ In order to avoid possible confusion we want to stress that, despite the similar name, a double extension is a priori completely unrelated to a Lie algebra with two central extension.
} 
and on the level of the invariant metric, to a double extension of any other kinematical Lie algebra that can be reached by a contraction. Again, all of this extended algebras have the same homogeneous space as the original algebra.

A busy reader or somebody only interested in the final results could consider to look at our summary in section 8 and figure 1 and the discussion in section 9 .

This paper is organized as follows. In section 2 we recall the reformulation of the Einstein-Hilbert action in its first-order form as a CS gauge theory. We will emphasize in particular the rôle of the Klein pair and the choice of invariant metric. Section 3 contains one of the main results of this work. We discuss the possible IW contractions of the (A)dS algebra to other three-dimensional kinematical algebras, their nontrivial central extensions, and finally present the CS action based on the twice trivially extended (A)dS algebra from which all other actions of the cube 1 can be obtained as a limit. In section 4 we define the concept of a double extension and show how the appearance of a metric for the three-dimensional kinematical algebras of the cube can be understood from this perspective. Section 5 discusses the three-dimensional kinematical algebras that do not arise as limits from the (A)dS algebra, which finishes our classification of kinematical CS theories. In section 6 we apply the double extension bootstrap of section 4 to higher dimensional kinematical algebras. Section 7 presents then a generalization of the cube of Bacry-Lévy-Leblond for metric Lie algebras. Following our summary and discussion in sections 8 and 9, respectively, we include in appendix A our conventions concerning Lie algebra contractions. In appendix B we discuss concepts regarding kinematical Lie algebras, homogeneous spacetimes, and Cartan connections. In appendix $\mathrm{C}$ we display the full action constructed in section 3 with its equations of motion and gauge transformations.

Before starting, a word on terminology: We stress throughout this work that the specification of a kinematical algebra $\mathfrak{k}$ without providing also a subalgebra $\mathfrak{b}$ for the Klein pair $(\mathfrak{k}, \mathfrak{b})$ is not enough to define the homogeneous spacetime it acts on. Nevertheless, our use of terminology will be more loose in the sense that we will often refer to the homogeneous spacetimes by their kinematical algebras. The reason for this is that often only this structure only is relevant for the problem at hand. The choice of subalgebra $\mathfrak{b}$ necessary for the Klein pair will be obvious from our choice of basis for $\mathfrak{k}$.

\section{Chern-Simons theory and homogeneous spacetimes}

We briefly review the reformulation of the Einstein-Hilbert action as a gauge theory of Chern-Simons type (for more details see [2]). In section 2.2 we will then frame the discussion in terms of homogeneous spacetimes. We argue that it is possible to write equations of motion for any three-dimensional spatially isotropic spacetime as classified in $[38,40]$ and explain what is necessary to also provide an action principle.

\subsection{Three-dimensional gravity as a Chern-Simons theory}

It is well-known that the first order form of the Einstein-Hilbert action in three dimensions can be written as a Chern-Simons theory $[1,2]$

$$
S_{\mathrm{CS}}[A]=\int\left\langle A \wedge d A+\frac{1}{3}[A, A] \wedge A\right\rangle,
$$

where a wedge product is understood in the commutator. 


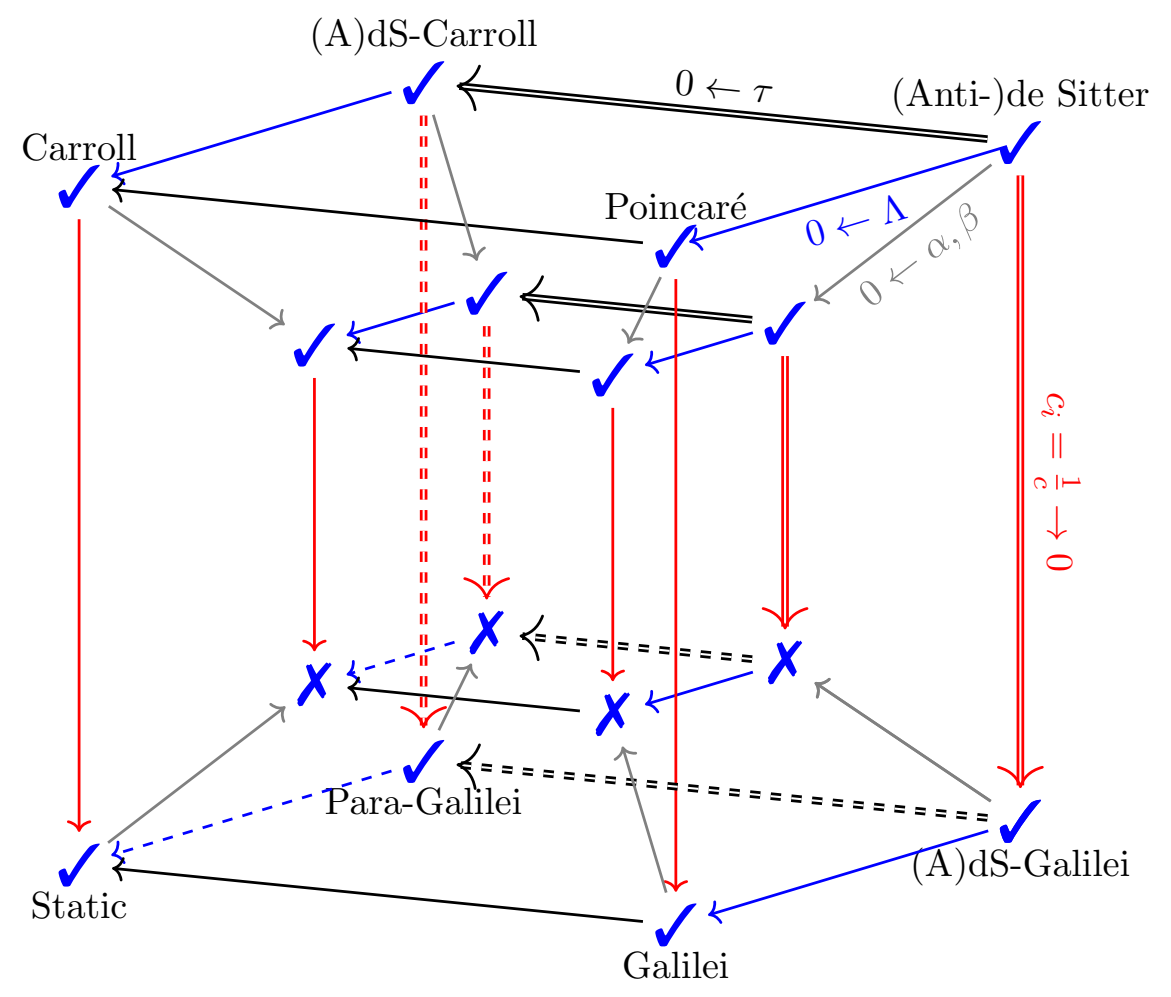

Figure 1. This tesseract describes the limits starting with the (anti-)de Sitter theories extended by two (trivial) central extensions. They are added such that they are nontrivial in the nonrelativistic $\left(c_{i} \rightarrow 0\right)$ limit and render all theories well-defined $(\boldsymbol{V})$ in the flat $(\Lambda \rightarrow 0)$ and ultrarelativistic $(\tau \rightarrow 0)$ limit. This spans the outer cube which is described in table 2. The $\alpha, \beta \rightarrow 0$ limit trivializes the central extensions and leads to the inner cube. The nonrelativistic theories are, unlike the carrollian, not well-defined anymore. This is due to degeneracy of their invariant metric as indicated by a cross $(\boldsymbol{X})$ and can be seen in table 1 . See also table 10 for a summary of the properties of the algebras appearing in the tesseract.

In the first order formulation of general relativity the fundamental fields are the vielbein $e$ and the dualized spin-connection $\omega$, which can be combined into the connection $A$ of the Chern-Simons theory

$$
A=e+\omega=e^{\mu} \hat{\mathrm{P}}_{\mu}+\omega^{\mu} \hat{\mathrm{J}}_{\mu} .
$$

The connection $A$ is then a Lie algebra valued one form with the Lie algebra given by $\mathfrak{a d s}=\mathfrak{s o}(2,2) \simeq \mathfrak{s l}(2, \mathbb{R}) \oplus \mathfrak{s l}(2, \mathbb{R}) \simeq \mathfrak{s o}(2,1) \oplus \mathfrak{s o}(2,1), \mathfrak{d} \mathfrak{s}=\mathfrak{s o}(3,1) \simeq \mathfrak{s l}(2, \mathbb{C})$ (where $\mathfrak{s l}(2, \mathbb{C})$ is understood as a real Lie algebra) and $\mathfrak{p o i}=\mathfrak{i s o}(2,1)$, for negative, positive and vanishing cosmological constant, respectively. These algebras are explicitly given by

$$
\begin{aligned}
& {\left[\hat{\mathrm{J}}_{\mu}, \hat{\mathrm{J}}_{\nu}\right]=\epsilon_{\mu \nu}{ }^{\rho} \hat{\mathrm{J}}_{\rho}} \\
& {\left[\hat{\mathrm{J}}_{\mu}, \hat{\mathrm{P}}_{\nu}\right]=\epsilon_{\mu \nu}{ }^{\rho} \hat{\mathrm{P}}_{\rho}} \\
& {\left[\hat{\mathrm{P}}_{\mu}, \hat{\mathrm{P}}_{\nu}\right]= \pm \Lambda^{2} \epsilon_{\mu \nu}{ }^{\rho} \hat{\mathrm{J}}_{\rho} .}
\end{aligned}
$$


The upper sign refers to $\mathfrak{a} \mathfrak{d s}$, the lower sign to $\mathfrak{d} \mathfrak{s}$ and $\mathfrak{p o i}$ is given by the $\Lambda \rightarrow 0$ limit. $^{5}$ Indices are raised and lowered with a mostly plus metric (our conventions are summarized in appendix A). In each case the Lie algebra corresponds to the isometry algebra of the maximally symmetric spacetime of the theory given by anti-de Sitter, de Sitter, and Minkowski space.

Having chosen a gauge algebra, the Chern-Simons theory is not completely defined without choosing a "trace", which was denoted by \langle\rangle in (2.1). We will call this symmetric, invariant, nondegenerate bilinear form on the Lie algebra an invariant metric where by invariance we mean that

$$
\mathrm{Z} \cdot\langle\mathrm{X}, \mathrm{Y}\rangle \equiv\langle[\mathrm{Z}, \mathrm{X}], \mathrm{Y}\rangle+\langle\mathrm{X},[\mathrm{Z}, \mathrm{Y}]\rangle=0
$$

for all Lie algebra elements X, Y, Z. ${ }^{6}$ For simple complex Lie algebras it is given up to a normalization by the Killing form. But, as discussed, ads is semisimple as is the complexification of $\mathfrak{d} \mathfrak{s}$ (as a real Lie algebra $\mathfrak{d} \mathfrak{s}$ is of course simple). Therefore, the invariant metric of the real Lie algebras is parametrized by two parameters $\mu$ and $\chi$

$$
\begin{aligned}
& \left\langle\hat{\mathrm{J}}_{\rho}, \hat{\mathrm{J}}_{\lambda}\right\rangle_{\chi}=\chi \eta_{\rho \lambda} \\
& \left\langle\hat{\mathrm{P}}_{\rho}, \hat{\mathrm{J}}_{\lambda}\right\rangle_{\mu}=\mu \eta_{\rho \lambda} \\
& \left\langle\hat{\mathrm{P}}_{\rho}, \hat{\mathrm{P}}_{\lambda}\right\rangle_{\chi}= \pm \Lambda^{2} \chi \eta_{\rho \lambda} .
\end{aligned}
$$

This is the most general invariant metric and it is nondegenerate for $\mu^{2} \mp \Lambda^{2} \chi^{2} \neq 0$. In particular, the metric is nondegenerate even in the $\Lambda \rightarrow 0$ limit provided $\mu \neq 0$, even though poi is not semisimple.

It is clear that this metric for poi cannot come from its Killing form, that is degenerate due to Cartan's criterion, which shows that semisimplicity is not a necessary criterion for the existence of an invariant metric. Rather, the poi algebra has the specific form of a double extension which guarantees the existence of an invariant metric [42, 43]. We will return to the topic of double extensions in section 4. For now let us note only that the double extension structure of the Poincaré algebra in three dimensions does not generalize to higher dimension. On the other hand, the invariant metric proportional to $\chi$, coming from the Killing form of the semisimple $(\mathfrak{a}) \mathfrak{d} \mathfrak{s}$ algebra, is generic.

Having defined a metric on the Lie algebra one can now plug the connection (2.2) into the Chern-Simons action (2.1) and use (2.3) and (2.5) to obtain

$$
\begin{aligned}
S_{\mathrm{CS}}[e, \omega] & =\int\left\langle 2 e \wedge R+\frac{1}{3}[e, e] \wedge e-d(\omega \wedge e)\right\rangle_{\mu}+\int\left\langle e \wedge T+\omega \wedge d \omega+\frac{1}{3}[\omega, \omega] \wedge \omega\right\rangle_{\chi} \\
& =S_{\mathrm{EH}}+S_{\mathrm{E} \chi}
\end{aligned}
$$

where the dualized curvature two-form is given by $R=d \omega+\frac{1}{2}[\omega, \omega]$ and the torsion twoform by $T=d e+[\omega, e]$. The first term, with coupling $\mu$, is proportional to the standard

\footnotetext{
${ }^{5}$ The above mentioned splitting of the ads algebra is made explicit by the basis change $\hat{\mathrm{L}}_{\mu}^{ \pm}=$ $\frac{1}{2}\left(\hat{\mathrm{J}}_{\mu} \pm \Lambda^{-1} \hat{\mathrm{P}}_{\mu}\right)$.

${ }^{6}$ We will often abuse terminology and refer to a degenerate invariant symmetric bilinear form by degenerate (invariant) metric. If not indicated otherwise, we mean by an invariant metric a nondegenerate bilinear form.
} 
Einstein-Hilbert action of general relativity in the first order formulation. The second "exotic" term with coupling $\chi$, has the interesting feature that it does not change the equations of motion [2], as we will now show explicitly

$$
\begin{aligned}
\delta S_{\mathrm{CS}}= & \int\langle 2 F \wedge \delta A\rangle-\int d\langle A \wedge \delta A\rangle \\
= & \int\langle(2 R+[e, e]) \wedge \delta e+2 T \wedge \delta \omega\rangle_{\mu}-\int d\langle e \wedge \delta \omega+\omega \wedge \delta e\rangle_{\mu} \\
& +\int\langle 2 T \wedge \delta e+(2 R+[e, e]) \wedge \delta \omega\rangle_{\chi}-\int d\langle e \wedge \delta e+\omega \wedge \delta \omega\rangle_{\chi} .
\end{aligned}
$$

Thus, in each case the Chern-Simons flatness condition

$$
F=d A+\frac{1}{2}[A, A]=0
$$

implies, for generic $\mu$ and $\chi$, the equations of motion

$$
R^{\lambda}=\mp \frac{\Lambda^{2}}{2} \epsilon_{\nu \rho}^{\lambda} e^{\nu} \wedge e^{\rho} \quad T^{\lambda}=0 .
$$

The resulting geometries are therefore quotients of three-dimensional Minkowski or (A)dS.

While the addition of the exotic term leaves the equations of motion inert, let us emphasize that this change of the action has repercussion on the thermodynamics of the solutions [44-46] and, consequently, might change the quantum theory [2]. We will therefore try to be as general as possible when providing invariant metrics for other Lie algebras.

We parametrize the gauge transformations $\delta A=d \varepsilon+[A, \varepsilon]$ by $\varepsilon=\zeta^{\mu} \hat{\mathrm{P}}_{\mu}+\lambda^{\mu} \hat{\mathrm{J}}_{\mu}$ which leads us to

$$
\delta e=d \zeta+[\omega, \zeta]+[e, \lambda], \quad \delta \omega=d \lambda+[\omega, \lambda]+[e, \zeta] .
$$

The remarkable feature that makes three dimensional gravity special is invariance of the action, up to a boundary term, under local Lorentz transformations and local translations. Diffeomorphisms $\xi$ are on-shell just gauge transformations given by $\varepsilon=\xi^{\mu} A_{\mu}$ [2].

Furthermore, one can construct a metric being invariant under local Lorentz transformations $\lambda$ by

$$
g=\eta_{\mu \nu} e^{\mu} \otimes e^{\nu}
$$

This quantity characterizes lorentzian geometries and will in general be lost in the kinematical limits. This metric on the tangent space of the spacetime $\mathcal{M}$ should not be confused with the earlier introduced invariant metric of the Lie algebra.

\subsection{Chern-Simons theories for three-dimensional homogeneous spacetimes}

Let us discuss the reformulation of Einstein gravity presented in the previous section from the point of view of homogeneous spacetimes; for a summary of relevant concepts consult appendix B. The three algebras $\mathfrak{a d s}, \mathfrak{d s}$, and poi are examples of a kinematical Lie algebra $\mathfrak{k}$. In order to define a homogeneous spacetime associated to a kinematical algebra $\mathfrak{k}$ we 
need in addition the choice of a subalgebra $\mathfrak{b} \subset \mathfrak{k}$ that determines the subalgebra of boosts and rotations. For all of the three algebras this subalgebra is the three-dimensional Lorentz algebra $\mathfrak{s o}(2,1)$ in equation $(2.3 \mathrm{a})$ generated by the elements $\left\{\hat{\mathrm{J}}_{\mu}\right\}$. Writing down the gauge connection $A$, the elements along the $\mathfrak{b}$ direction are associated with the spin connection $\omega$, whereas elements along the remaining directions $\mathfrak{k} / \mathfrak{b}$ are associated with the dreibein $e$. Choosing an invariant metric one can then write the CS action (2.1) for the connection A. Projecting the equation of motion (2.9) along the $\mathfrak{b}$ direction yields the curvature equation, while projecting along the remaining generators $\mathfrak{k} / \mathfrak{b}$ yields the torsion of the spacetime. Since the homogeneous spacetimes obtained from the pair $(\mathfrak{k}, \mathfrak{s o}(2,1))$, with $\mathfrak{k}$ being any of $\mathfrak{a} \mathfrak{d} \mathfrak{s}, \mathfrak{d} \mathfrak{s}$, or $\mathfrak{p o i}$, can be equipped with a lorentzian structure $\eta_{\mu \nu}$ we can write down the spacetime metric $g$ according to equation (2.12).

Framing the derivation of the last section in this way, we see that it is possible to write down equations of motion for all of the three-dimensional homogeneous spacetimes classified in [40]. One simply replaces the Klein pair of, say, $(\mathfrak{a} \mathfrak{d s}, \mathfrak{s o}(2,1))$ by the corresponding Klein pair $(\mathfrak{k}, \mathfrak{b})$. The equations of motion (2.9) provide a theory for the gauge field $A$ of the underlying symmetry algebra. More precisely, the connection $A$ is the Cartan connection for a Cartan geometry. These geometries describe manifolds that are locally modeled on a particular homogeneous spacetime, in the same way (pseudo-)riemannian manifolds are locally modeled on euclidean (Minkowski) spacetime (for more details and references see appendix B). Of course, the equation of motion (2.9) tells us that, on-shell, all solutions are quotients of the model homogeneous spacetimes, i.e., the Cartan geometry is flat.

Since a generic homogeneous spacetime does not allow for a lorentzian structure $\eta_{\mu \nu}$, equation (2.12) is replaced by the corresponding carrollian, galilean or static structure. With the appropriate replacements one can write down a Cartan connection and the equations of motion $F=0$ for all higher-dimensional kinematical algebras.

However, there is an additional nontrivial ingredient that is needed to construct the CS action, which is the existence of an invariant metric on the kinematical Lie algebra. In fact, in addition to the choice of homogeneous spacetime this is the only nontrivial (local) ingredient in order to define a CS theory.

For the (semi)simple algebras $(\mathfrak{a}) \mathfrak{d} \mathfrak{s}$ the existence of this metric is guaranteed, while for poi it is only due to its special form as a double extension; we will return to this point in section 4 . In contrast, most kinematical algebras $\mathfrak{k}$ on which the spacetimes appearing in the classification of [40] are based, do not allow for an invariant metric. In order to obtain a CS theory for these kinematical algebras we will have to extend these algebras, i.e., we will have to add additional generators and corresponding fields to the gauge connection $A$ such that the resulting algebra can be endowed with an invariant metric. These extensions will be modeled to have the structure of a double extension with the minimal number of additional generators. This choice guarantees, e.g., compared to a deformation, that the geometries do not loose their kinematical structure and interpretation. Note that this is a choice and there might be other ways to realize CS theories for these homogeneous spacetimes. Nevertheless, using our approach we will be able to construct CS theories for all three-dimensional homogeneous spacetimes. 


\section{Kinematical limits of three-dimensional gravity}

In the previous section we have reviewed the Chern-Simons formulation of threedimensional gravity and subsequently generalized the discussion to include Chern-Simons theories based on any three-dimensional homogeneous spacetime provided they admit an invariant metric on their Lie algebra.

Kinematical spacetimes have been classified in the seminal work of Bacry and LévyLeblond [38, 39] with a recent generalization presented in [40]. Among these spacetimes there is a class that arises as a limit from (A)dS space. In this section we focus on this family. The remaining so-called non-contracting kinematical spacetimes will be dealt with in section 5 .

For interesting Chern-Simons theories that go beyond or generalize the kinematical setup, see, e.g., [35-37, 47-49].

\subsection{Kinematical limits}

Since the (semi)simple (a)d $\mathfrak{d}$ algebras cannot arise as nontrivial contractions, ${ }^{7}$ they present a natural starting point in the search for limits. We will call a limit or action well-defined if the invariant metric remains nondegenerate after the limit, which means that each field has a kinetic term $\langle A \wedge d A\rangle$ in the CS action.

In the following, it will be convenient to introduce a time-space splitting of the $(\mathfrak{a}) \mathfrak{d} \mathfrak{s}$ Lie algebra (2.3). We assume the following conventions for the generators of rotations $\mathrm{J}$, dualized boosts $\mathrm{B}_{a}$, time translation $\mathrm{H}$, and spatial translations $\mathrm{P}_{a}$

$$
\mathrm{J} \equiv \hat{\mathrm{J}}_{0} \quad \mathrm{~B}_{a} \equiv \hat{\mathrm{J}}_{a} \quad \mathrm{H} \equiv \hat{\mathrm{P}}_{0} \quad \mathrm{P}_{a} \equiv \hat{\mathrm{P}}_{a},
$$

where $a=1,2$ (for a summary of our conventions see appendix A.1). The subalgebra $\mathfrak{b}$ of the kinematical algebra is always assumed to be generated by the set $\left\{\mathrm{J}, \mathrm{B}_{a}\right\}$.

Besides the already introduced flat or vanishing cosmological constant limit parametrized by $\Lambda \rightarrow 0$ we have two further independent options to introduce contraction parameters when we restrict to kinematical spacetimes [38] (which is also true under less restrictive conditions [40]):

- The nonrelativistic or galilean limit corresponds intuitively to the limit where the light cone opens up and is parametrized by sending the speed of light to infinity. We will introduce a contraction parameter that is the inverse of the speed of light $c_{i} \equiv \frac{1}{c}$ so that we can send all the limits uniformly to zero. One ends up with (A)dS galilean spacetimes, also known as Newton-Hooke spacetimes or expanding/oscillating universes [38]. For the case of vanishing cosmological constant, the $c_{i} \rightarrow 0$ limit leads to the galilean spacetime.

\footnotetext{
${ }^{7}$ This follows from the fact that there exists for any Lie algebra contraction an inverse operation given by a Lie algebra deformation. This implies that a Lie algebra that is the result of a nontrivial contraction should allow for deformations. However, semisimple Lie algebras, like the $(\mathfrak{a}) \mathfrak{d} \mathfrak{s}$ algebras, cannot be deformed (they are rigid) and can therefore not arise from a limit (see, e.g., $[50,51]$ for further details).
} 
- The ultrarelativistic or carrollian limit corresponds to the limit where the light cone closes. It is an independent and inequivalent contraction parametrized by $\tau$ which we also send to zero in the limit. This leads to the (A)dS-carrollian spacetimes, which were called para-Poincaré and inhomogeneous $\mathrm{SO}(4)$ in [38]. Taking both $\Lambda \rightarrow 0$, $\tau \rightarrow 0$ limits leads to the carrollian spacetime. These carrollian spacetimes are null surfaces of (A)dS and Minkowski space [40] (see also [52]).

Notice that seen as an abstract Lie algebra the AdS-Carroll algebra is isomorphic to the Poincaré algebra. However, the associated Klein pairs, and consequently the associated homogeneous spacetimes, differ due to a different choice of subalgebra $\mathfrak{b}$ for the same kinematical algebra. This example emphasizes the physical importance of considering Klein pairs instead of kinematical algebras only.

- Keeping the cosmological constant but sending $\tau, c_{i} \rightarrow 0$ (in any order) leads to the so-called para-Galilei algebra. As in the above case, the para-Galilei algebra is isomorphic to the Galilei algebra. The homogeneous spacetimes associated to the two algebras are different due to a different choice of subalgebra $\mathfrak{b}$. We want to mention the following subtlety: since the boosts $\mathrm{B}_{a}$ in the para-Galilei algebra do not act effectively on $\mathrm{P}_{a}, \mathrm{H}$, they can be quotiented out of the algebra (cf. appendix B). Consequently, the homogeneous spacetime of the para-Galilei algebra is the same static aristotelian spacetime as for the static algebra, cf. below. ${ }^{8}$

- Sending all contraction parameters to zero $\Lambda, \tau, c_{i} \rightarrow 0$ leads to the static algebra with all brackets identically zero, except for the commutators of angular momentum with boosts and spatial momentum. The static algebra belongs to the class of aristotelian kinematical algebras, which we will analyze in more detail in section 5.3.

These limits can be implemented in the following way. Starting from the generators (3.1) that are appropriate for the relativistic algebras we will change to a new basis by a threeparameter $\left(c_{i}, \tau, \Lambda\right)$ family of linear transformations $g_{c_{i}, \tau, \Lambda}$

$$
g_{\Lambda, \tau, c_{i}} \mathrm{~J}=\mathrm{J} \quad g_{\Lambda, \tau, c_{i}} \mathrm{~B}_{a}=\left(\tau c_{i}\right)^{-1} \mathrm{~B}_{a} \quad g_{\Lambda, \tau, c_{i}} \mathrm{P}_{a}=\left(\Lambda c_{i}\right)^{-1} \mathrm{P}_{a} \quad g_{\Lambda, \tau, c_{i}} \mathrm{H}=(\Lambda \tau)^{-1} \mathrm{H},
$$

simultaneously transforming the metric $(2.5)$

$$
g_{\Lambda, \tau, c_{i}} \mu=(\Lambda \tau)^{-1} \mu \quad g_{\Lambda, \tau, c_{i}} \chi=\chi,
$$

where we have for completeness also added the cosmological constant that was already used in section 2. The resulting generators on the right hand side are taken to be the generators for the contracted algebras. The replacements of algebra (2.3) and invariant metric (2.5) lead to table 1 . The invariant metric is nondegenerate for

$$
c_{i}\left(\mu^{2} \mp \Lambda^{2} \tau^{2} \chi^{2}\right) \neq 0 .
$$

\footnotetext{
${ }^{8}$ For the CS theories this question of equivalence is more subtle and the reason we keep the paragalilean theory.
} 


\begin{tabular}{|lr|}
\hline & $\left.(\mathfrak{a}) \mathfrak{d} \mathfrak{s}_{(\mp}\right)$ \\
\hline$\left[\mathrm{J}, \mathrm{B}_{a}\right]$ & $\epsilon_{a m} \mathrm{~B}_{m}$ \\
{$\left[\mathrm{~J}, \mathrm{P}_{a}\right]$} & $\epsilon_{a m} \mathrm{P}_{m}$ \\
{$\left[\mathrm{~B}_{a}, \mathrm{~B}_{b}\right]$} & $-\epsilon_{a b} \tau^{2} c_{i}^{2} \mathrm{~J}$ \\
{$\left[\mathrm{~B}_{a}, \mathrm{H}\right]$} & $-\epsilon_{a m} \tau^{2} \mathrm{P}_{m}$ \\
{$\left[\mathrm{~B}_{a}, \mathrm{P}_{b}\right]$} & $-\epsilon_{a b} c_{i}^{2} \mathrm{H}$ \\
{$\left[\mathrm{H}, \mathrm{P}_{a}\right]$} & $\pm \epsilon_{a m} \Lambda^{2} \mathrm{~B}_{m}$ \\
{$\left[\mathrm{P}_{a}, \mathrm{P}_{b}\right]$} & $\mp \epsilon_{a b} \Lambda^{2} c_{i}^{2} \mathrm{~J}$ \\
\hline
\end{tabular}

\begin{tabular}{|lr|}
\hline & $(\mathfrak{a}) \mathfrak{d}_{(\mp)}$ \\
\hline$\langle\mathrm{H}, \mathrm{J}\rangle$ & $-\mu$ \\
$\left\langle\mathrm{P}_{a}, \mathrm{~B}_{b}\right\rangle$ & $c_{i}^{2} \mu \delta_{a b}$ \\
$\langle\mathrm{~J}, \mathrm{~J}\rangle$ & $-\chi$ \\
$\left\langle\mathrm{B}_{a}, \mathrm{~B}_{b}\right\rangle$ & $c_{i}^{2} \tau^{2} \chi \delta_{a b}$ \\
$\langle\mathrm{H}, \mathrm{H}\rangle$ & $\mp \Lambda^{2} \tau^{2} \chi$ \\
$\left\langle\mathrm{P}_{a}, \mathrm{P}_{b}\right\rangle$ & $\pm c_{i}^{2} \Lambda^{2} \chi \delta_{a b}$ \\
\hline
\end{tabular}

Table 1. The (anti-)de Sitter spacetimes (left) and their most general invariant metric (right). The upper signs correspond to $\mathfrak{a} \mathfrak{d s}$ and the lower to $\mathfrak{d} \mathfrak{s}$. The invariant metric remains nondegenerate when $c_{i}\left(\mu^{2} \mp \Lambda^{2} \tau^{2} \chi^{2}\right) \neq 0$.

From the degeneracy criterion (3.4) we see that the invariant metric is non-singular in the limit $\tau \rightarrow 0$ if $\mu \neq 0$. Since also all Lie brackets are well-defined in this limit, this means that the ultrarelativistic limit $\tau \rightarrow 0$ of the Chern-Simons action (2.1) is well-defined. This has first been shown for the $\Lambda=0$ case in [31,32], to the best of our knowledge.

On the other hand, in the nonrelativistic limit the bilinear form is degenerate regardless of the choice of $\mu$ and $\chi$; the treatment of this degeneracy is the subject of section 3.3. The different limits relating the kinematic theories presented in this section are summarized in the inner cube of the tesseract of figure 1. The check marks on the upper face of the inner cube signify that the limiting procedure leads to a nondegenerate invariant metric and thus to a well-defined theory.

A comment concerning the contractions and invariant metrics is in order. It might happen that a contraction leads to a degenerate bilinear form even though the contracted Lie algebra permits an invariant metric. A straightforward example is the contraction of an arbitrary algebra with invariant metric to an abelian one; a contraction that is always available. Any abelian algebra permits an invariant metric, still the contracted invariant metric might be degenerate.

The situation for the case at hand is that, using the contractions of table 1, we arrive at the most general invariant metric for the lorentzian and carrollian theories. For the Galilei, (A)dS-Galilei, para-Galilei and static case we have the freedom to add $\langle\mathrm{H}, \mathrm{H}\rangle=\chi_{\mathrm{H}}$, but the invariant metric is still degenerate.

\subsection{Central extensions of kinematical algebras}

We saw in the last subsection that the nonrelativistic theories do not inherit a nondegenerate metric in the limit $c_{i} \rightarrow 0$. However, this should not come as a surprise as it is straightforward to show that none of these algebras permits an invariant metric.

In order to see this it is enough to realize that the center $Z(\mathfrak{g})$ of any Lie algebra $\mathfrak{g}$ that allows for an invariant metric is orthogonal to the first derived ideal $[\mathfrak{g}, \mathfrak{g}]$. This is implied by the following chain of equivalences

$$
[Z(\mathfrak{g}), \mathfrak{g}]=0 \quad \Longleftrightarrow \quad\langle[Z(\mathfrak{g}), \mathfrak{g}], \mathfrak{g}\rangle=0 \quad \Longleftrightarrow \quad\langle Z(\mathfrak{g}),[\mathfrak{g}, \mathfrak{g}]\rangle=0,
$$


where the first arrow follows from nondegeneracy of the invariant metric and the second from invariance. The last equation gives us the simple necessary criterion

$$
\operatorname{dim} \mathfrak{g}=\operatorname{dim}[\mathfrak{g}, \mathfrak{g}]+\operatorname{dim} Z(\mathfrak{g})
$$

for the existence of an invariant metric for a given algebra $\mathfrak{g}$. This is trivially fulfilled for semisimple Lie algebras, where $\mathfrak{g}=[\mathfrak{g}, \mathfrak{g}]$, and abelian Lie algebras, since $\mathfrak{g}=Z(\mathfrak{g})$.

Applying equation (3.6) to the nonrelativistic algebras we find that the criterion is not obeyed as all of them have no or (as in the case of the static algebra) not enough central elements. From this we conclude that further generators, e.g, in the form of central elements have to be added to the nonrelativistic algebras if we require these algebras to have a nondegenerate invariant metric. Our aim in section 3.3 will be to include these central extensions before taking the nonrelativistic limit, in such a way that after the contraction the resulting algebras have a well-defined metric; partial results of this contraction procedure (sometimes implicitly) can be found in [31, 33-35, 37, 48]. Before we turn to the contraction procedure we study all possible central extensions of the kinematical algebras and their invariant metrics that appear in the cube of Chern-Simons theories. Central extensions are trivial if they can be removed by a redefinition of the basis elements. Nontrivial central extensions of the algebra are in one-to-one correspondence with the elements of the second cohomology group of the Lie algebra (for details cf., e.g., [53]). We mention that (semi)simple Lie algebras can only have trivial central extensions.

The (A)dS-Galilei algebra and its flat limit, the Galilei algebra, allow for three central extensions [54]

$$
[\mathrm{J}, \mathrm{H}]=\mathrm{Z}_{\mathrm{JH}} \quad\left[\mathrm{B}_{a}, \mathrm{P}_{b}\right]=\epsilon_{a b} \mathrm{M} \quad\left[\mathrm{B}_{a}, \mathrm{~B}_{b}\right]=\epsilon_{a b} \mathrm{~S} \quad\left[\mathrm{P}_{a}, \mathrm{P}_{b}\right]= \pm \Lambda^{2} \epsilon_{a b} \mathrm{~S}
$$

of which only the "mass" M generalizes to higher dimension. The Galilei algebra with the central extension M included is called the Bargmann algebra. Its importance lies in the fact that its corresponding Lie group is isomorphic to the symmetry group of galilean systems as described, e.g., by the Schrödinger equation. The three-dimensional Galilei algebra with both central charges M, S is sometimes called extended Bargmann algebra.

The Lie algebra central extension $[\mathrm{J}, \mathrm{H}]=\mathrm{Z}_{\mathrm{JH}}$ does not exponentiate to an extension of the group [54] and is therefore often omitted. Furthermore, from the point of view that $\mathrm{H}$ should be a scalar under rotations this central extension seems obscure. Let us check the necessary criterion (3.6) for the Galilei algebra again but now with the central extensions (3.7) included. We find that the dimension of the first derived ideal $\left\{\mathrm{B}_{a}, \mathrm{P}_{a}, \mathrm{M}, \mathrm{S}, \mathrm{Z}_{\mathrm{JH}}\right\}$ and the dimension of the center $\left\{\mathrm{S}, \mathrm{Z}_{\mathrm{JH}}, \mathrm{M}\right\}$ do not add up to the dimension of the algebra, thus disallowing an invariant metric, unless one of the central extensions is dropped. Indeed, the presence of $\mathrm{Z}_{\mathrm{JH}}$ renders any invariant metric degenerate, whereas the other two central extensions are needed for a nondegenerate metric. We will see this in more detail in sections 3.3 and 4.3 .

For para-Galilei we merely exchange $\mathrm{B} \leftrightarrow \mathrm{P}$ in (3.7) and send $\Lambda \rightarrow 0$, so infinitesimally it also has three central extensions given by

$$
[\mathrm{J}, \mathrm{H}]=\mathrm{Z}_{\mathrm{JH}} \quad\left[\mathrm{B}_{a}, \mathrm{P}_{b}\right]=\epsilon_{a b} \mathrm{M} \quad\left[\mathrm{P}_{a}, \mathrm{P}_{b}\right]=\epsilon_{a b} \mathrm{Z}_{\mathrm{P}} .
$$


For the relativistic algebras, i.e., the upper face of the cube, we do not need central extensions in order to get an invariant metric, as we showed in the last section. However, in order to be complete we mention the possible central extensions for these algebras as well. The Lie algebras of the Poincaré and (A)dS-Carroll spacetimes permit, like their higher dimensional analogs, no nontrivial central extension. The flat Carroll spacetime permits, again only in $2+1$ dimensions, three central extensions given by

$$
\left[\mathrm{B}_{a}, \mathrm{P}_{b}\right]=\delta_{a b} \tilde{\mathrm{Z}}_{\mathrm{BP}} \quad\left[\mathrm{B}_{a}, \mathrm{~B}_{b}\right]=\epsilon_{a b} \mathrm{Z}_{\mathrm{B}} \quad\left[\mathrm{P}_{a}, \mathrm{P}_{b}\right]=\epsilon_{a b} \mathrm{Z}_{\mathrm{P}} .
$$

Adding any combination of these to the algebra renders the metric degenerate.

Finally, the static Lie algebra has five nontrivial central extensions (see, e.g., [55]) given by combining all of the above mentioned to

$$
[\mathrm{J}, \mathrm{H}]=\mathrm{Z}_{\mathrm{JH}} \quad\left[\mathrm{B}_{a}, \mathrm{P}_{b}\right]=\epsilon_{a b} \mathrm{M}+\delta_{a b} \tilde{\mathrm{Z}}_{\mathrm{BP}} \quad\left[\mathrm{B}_{a}, \mathrm{~B}_{b}\right]=\epsilon_{a b} \mathrm{Z}_{\mathrm{B}} \quad\left[\mathrm{P}_{a}, \mathrm{P}_{b}\right]=\epsilon_{a b} \mathrm{Z}_{\mathrm{P}} .
$$

Applying criterion (3.6) again, we see that it is obeyed if only one central extension is added. In fact, the only central extensions that allow the extended static algebra to have an invariant metric are either $\tilde{Z}_{\mathrm{BP}}$ or $\mathrm{M}$. We will recover the latter of these extensions when studying the contraction of the centrally extended (A)dS algebra which is the topic of the following section.

\subsection{The tesseract of Chern-Simons theories}

In the previous section we have not dealt with the ( $\mathfrak{a}) \mathfrak{d} \mathfrak{s}$ algebra, as it is a (semi)simple algebra with trivial second cohomology group. Thus, all central extensions of the (a)d $\mathfrak{d}$ algebra can be eliminated by a redefinition of the generators. However, trivial central extension may turn nontrivial under contractions. This will allow us to obtain a nondegenerate metric for the nonrelativistic algebras.

We extend the $(\mathfrak{a}) \mathfrak{d} \mathfrak{s}$ algebra by two $\mathfrak{u}(1)$ algebras with generators $\mathbf{S}$ and M. The Lie brackets of the extended algebra are summarized in table 2 (for details see appendix A.2). Let us emphasize that it is essential to add these two central extensions, as well as to consider them as independent elements of the vector space.

Notice that in the limit $\alpha, \beta \rightarrow 0$ which "turns off" the central extensions the brackets and metric of table 1 are reproduced. Furthermore, the central extensions generated by $\mathrm{M}$ and $\mathrm{S}$ are trivial so that the algebra displayed in table 2 is related to the previous one by a shift of generators. Since we added two new $\mathfrak{u}(1)$ generators we are free to choose an arbitrary nondegenerate symmetric bilinear form as invariant metric for these generators providing us with three parameters $\mu_{\mathrm{S}}, \mu_{\mathrm{M}}, \mu_{\mathrm{MS}}$. The other components of the metric are then fixed by the above mentioned shifts of generators. For an explicit display of the necessary replacements consult appendix A.2.

As before, the algebra is well-defined for all contractions $\Lambda, \tau, c_{i}$ but in contrast to the non-extended algebra of table 1 the invariant metric is now nondegenerate if both of the following conditions are fulfilled:

$$
\begin{array}{r}
c_{i}^{4}\left(\mu^{2} \mp \Lambda^{2} \tau^{2} \chi^{2}\right)+\alpha^{2} \beta^{2} \mu_{\mathrm{MS}}^{2} \mp \beta^{4} \Lambda^{2} \tau^{2} \mu_{\mathrm{S}}^{2}+2 c_{i}^{2} \beta\left(\alpha \mu \mu_{\mathrm{MS}} \mp \beta \Lambda^{2} \tau^{2} \chi \mu_{\mathrm{S}}\right) \neq 0 \\
\left(\mu_{\mathrm{S}} \mu_{\mathrm{M}}-\mu_{\mathrm{MS}}^{2}\right) \neq 0 .
\end{array}
$$




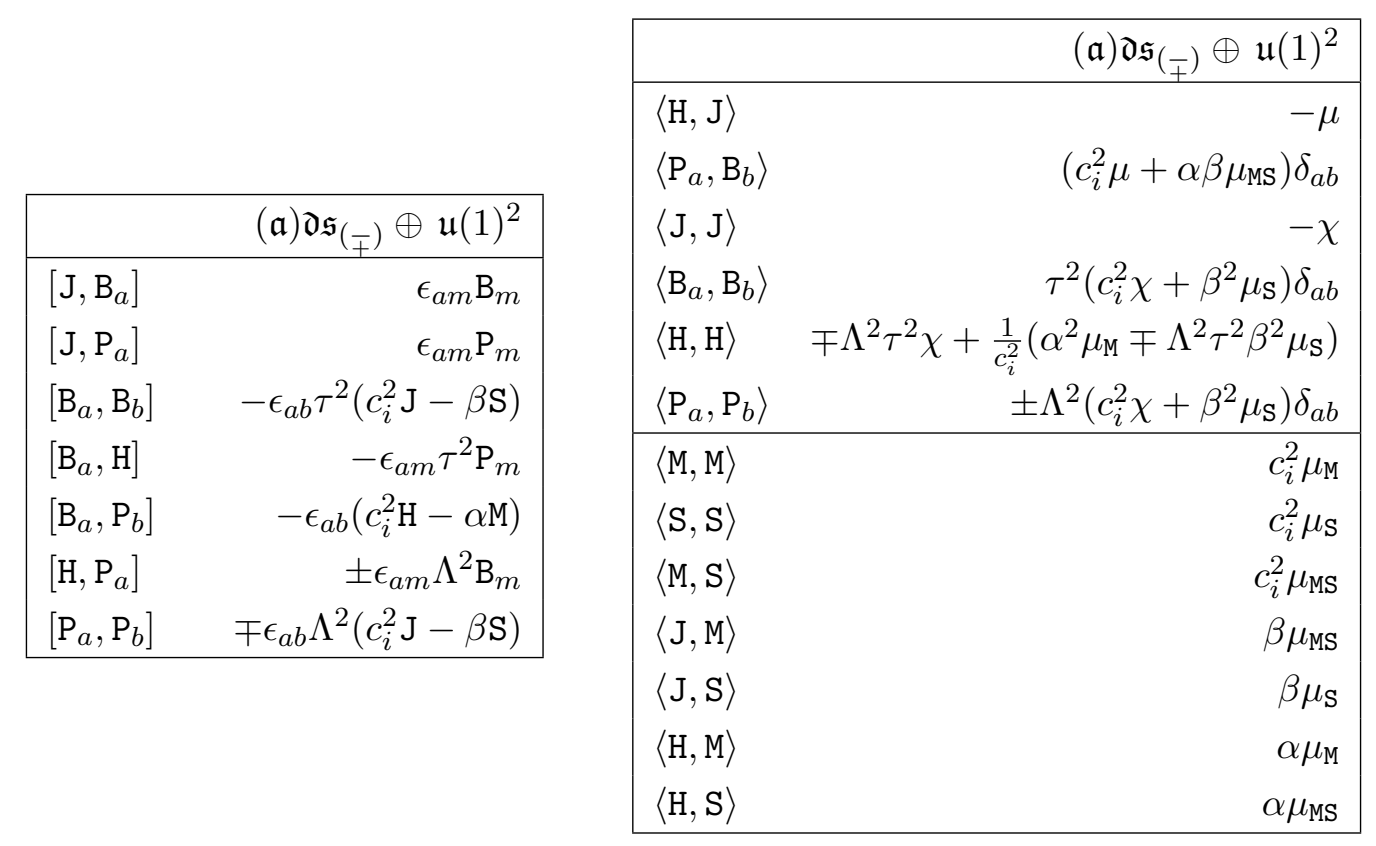

Table 2. Rescaled and trivially centrally extended (a) d s algebra (left) and invariant metric (right). For a well-defined nonrelativistic limit of the invariant metric we demand that $\alpha=\beta$ and $\mu_{\mathrm{M}}=$ $\pm \Lambda^{2} \tau^{2} \mu_{\text {s }}$. Under these conditions the limits to all theories are well-defined and lead to the outer cube of the tesseract of figure 1. The upper sign represents AdS, the lower dS.

Let us discuss the limits separately:

- For the relativistic theories with $c_{i} \neq 0$ already the non-extended metric of table 1 was nondegenerate. Furthermore, the central elements M, S are trivial from the point of view of these algebras. ${ }^{9}$

Consistent with this we see from (3.11a) that it is possible to turn off the central extensions by setting $\alpha=\beta=0$ while keeping the metric nondegenerate. This limit also turns off the metric elements "coupling" the two $\mathfrak{u}(1)$ generators to the remaining generators of the kinematical algebras. The resulting Chern-Simons theories therefore consists of two decoupled parts: one term associated to the respective relativistic kinematical algebra, the other one being two $\mathfrak{u}(1)$ Chern-Simons actions coupled to each other depending on the choice of $\mu_{\mathrm{MS}}$.

- The algebra obtained in the nonrelativistic limit is again the (A)dS-Galilei algebra for $\Lambda \neq 0$, or the Galilei algebra with $\Lambda=0$, with the two nontrivial central extensions denoted by $\mathrm{M}$ and $\mathrm{S}$ in (3.7). As mentioned above, precisely these two central extensions are needed in order to obtain a nondegenerate metric $[33,34] .{ }^{10}$ Consequently, equation (3.11a) shows that the invariant metric is now nondegenerate given that

\footnotetext{
${ }^{9}$ Although we saw before that the flat Carroll algebra allows for nontrivial central extensions the extension by $\mathrm{M}$ which is the only one surviving the limit $\Lambda, \tau \rightarrow 0$ is not the same as the extension by $\tilde{\mathrm{Z}}_{\mathrm{BP}}$ in equation (3.9).

${ }^{10}$ We note in passing that the AdS-Galilei algebra is decomposable, in a similar fashion as ads decomposes into $\mathfrak{s l}(2) \oplus \mathfrak{s l}(2)[34]$ (see also [48]).
} 
the central extensions are not turned off, $\alpha, \beta \neq 0$. However, due to our choice of parametrization the metric element $\langle\mathrm{H}, \mathrm{H}\rangle$ generically blows up in the nonrelativistic limit. In order to avoid this, we set

$$
\alpha=\beta \quad \mu_{\mathrm{M}}= \pm \Lambda^{2} \tau^{2} \mu_{\mathrm{S}}
$$

thus restricting to a four-parameter family of possible metrics. The invariant metric of the limit is the most general one if we add the term

$$
\langle\mathrm{H}, \mathrm{H}\rangle=\chi_{\mathrm{H}}
$$

which does not arise from the limit.

- The static algebra and its corresponding metric are obtained, again demanding (3.12), in the limit $\Lambda, c_{i}, \tau \rightarrow 0$. The metric is nondegenerate provided that $\alpha, \mu_{\mathrm{MS}} \neq 0$. From the two possible central extensions $\mathrm{M}$ and $\tilde{Z}_{\mathrm{BP}}$ that allow the static algebra to have a nondegenerate metric the contraction procedure realizes the former option. The central extension S trivializes. Notice, however, that it is not possible to decouple the field along the S direction while preserving a nondegenerate metric. The action based on the metric and algebra of table 2 in the static limit $\Lambda, \tau, c_{i} \rightarrow 0$ thus describes the aristotelian spacetime of the nontrivially centrally extended static algebra coupled to a $\mathfrak{u}(1)$ field. ${ }^{11}$

We have thus succeeded in providing a nondegenerate metric - and therefore also welldefined Chern-Simons theories - for all theories based on kinematical spacetimes that can be obtained from the three-dimensional (A)dS spacetimes as a limit. The situation is summarized in the tesseract of Chern-Simons theories in figure 1. The inner cube of the tesseract corresponds to the limit in which the (from the (A)dS perspective) trivial central extensions M, S are turned off. In this case, only the theories based on the relativistic algebras are well-defined, as designated by check marks on the respective corners. On the other hand, with the help of the two central extensions all algebras can be equipped with nondegenerate metrics.

\subsection{The most general action}

Based on the algebra and invariant metric for the trivially centrally-extended (a)d $\mathfrak{a}$ algebra of table 2 we are now able to construct the Chern-Simons action (2.1) that allows to obtain all other theories of the tesseract of figure 1 by taking various limits. ${ }^{12}$

The gauge field for this action

$$
A=A^{(0)}+A^{\left(\mathfrak{u}(1)^{2}\right)}
$$

\footnotetext{
${ }^{11}$ This means this is strictly speaking not the theory with solely the $\mathrm{M}$ central extension we discussed in section 3.2, which is due to our choice of contraction and invariant metric.

${ }^{12}$ This action should be considered the most general one given the most general invariant metric and given our construction, which identifies the vielbein and the spin-connection (which we choose in the case of symmetric spaces to be based on the canonical connection of the symmetric space, see section XI.3 in [56]) according to the underlying (symmetric) homogeneous spacetime. For a complementary perspective we refer to [57-60] and the discussions in [61].
} 
is given by a sum of the $\mathfrak{s o}(2,2)$ or $\mathfrak{s o}(3,1)$ gauge field $(2.2)$ denoted by $A^{(0)}$ and gauge field $A^{\left(\mathfrak{u}(1)^{2}\right)}$ with generators $\mathrm{S}$ and M. Written explicitly we have

$$
A=h \mathrm{H}+p^{a} \mathrm{P}_{a}+j \mathrm{~J}+b^{a} \mathrm{~B}_{a}+s \mathrm{~S}+m \mathrm{M} .
$$

The most general action can then be separated into three parts

$$
S=S_{\mathrm{CS}}[A]=S_{\mathrm{CS}}\left[A^{(0)}\right]+S_{\mathrm{CS}}\left[A^{\left(\mathfrak{u}(1)^{2}\right)}\right]+S_{\mathrm{I}}^{(\alpha, \beta)} .
$$

The action $S_{\mathrm{CS}}\left[A^{(0)}\right]$ contains contributions of the fields $h, p^{a}, j, b^{a}$ with the invariant metric taken to be the one given in table 2 with $\alpha, \beta \rightarrow 0$. When written in terms of $e$ and $\omega$ this is the same as (2.6). The second term is the abelian Chern-Simons action based on $A^{\left(\mathfrak{u}(1)^{2}\right)}=s \mathrm{~S}+m \mathrm{M}$, explicitly given by

$$
S_{\mathrm{CS}}\left[A^{\left(\mathfrak{u}(1)^{2}\right)}\right]=\int\left\langle A^{\left(\mathfrak{u}(1)^{2}\right)} \wedge d A^{\left(\mathfrak{u}(1)^{2}\right)}\right\rangle=c_{i}^{2} \int\left(\mu_{\mathrm{S}} s \wedge d s+\mu_{\mathrm{M}} m \wedge d m+2 \mu_{\mathrm{MS}} m \wedge d s\right) .
$$

The term $S_{\mathrm{I}}^{(\alpha, \beta)}$ contains all the fields together with the $\alpha, \beta$ dependent terms of the invariant metric in table 2 , that induce couplings between the $\mathfrak{u}(1)$ fields and the fields associated to the vielbein $e$ and the spin-connection $\omega$.

Even though specifying the Lie algebra and the invariant metric is sufficient to extract the equations of motion and gauge transformations, we still present them fully decomposed and for completeness in appendix C.

\section{Double extensions}

The fact that the Poincare algebra in $D+1$ spacetime dimensions iso $(D, 1)$ does not have a nondegenerate invariant bilinear metric has been one of the obstacles for attempts of constructing a theory of gravity invariant under the full Poincare group. ${ }^{13}$ This is related to $\mathfrak{i s o}(D, 1)$ not being semisimple, in contrast to the (A)dS algebra in $D+1$ dimensions. The magic of three dimensions, as observed by Witten in his seminal paper [2], lies in the observation that only in this case it is possible to find a metric for $\mathfrak{i s o}(2,1)$ although this algebra is still not semisimple. As all other magic phenomena this one too does not defy comprehension, in this particular case coming under the mundane term double extended algebra.

An algebra belonging to the class of double extensions comes automatically with a nondegenerate invariant metric. In the last sections we have seen a number of examples already in the form of the above mentioned $i \mathfrak{s o}(2,1)$, but also the Carroll algebras and the centrally extended galilean algebras belong to this class. In fact, any Lie algebra with a nondegenerate invariant metric that is not a direct sum of semisimple and abelian algebras is a double extension; we will make this statement more precise below.

In the following we will first introduce the concept of double extensions in section 4.1 and present the structure theorem of metric Lie algebras by Medina and Revoy. In sections 4.2 and 4.3 we will then show how the existence of invariant metrics for the kinematical algebras appearing in the tesseract of Chern-Simons theories can be understood from the perspective of double extensions.

\footnotetext{
${ }^{13}$ Nevertheless, there exist higher rank invariant forms on the Poincaré algebra that can be used to construct Chern-Simons theories in dimensions higher than three, see, e.g., [62-64].
} 


\subsection{Definition of double extensions}

In our definition of double extensions we will follow $[42,43]$ but stick to a discussion in terms of basis elements. For a basis-independent definition consult the original works.

Suppose $\left(\mathfrak{g},\langle,\rangle_{\mathfrak{g}}\right)$ is a Lie algebra with nondegenerate invariant metric, i.e., we have the relations

$$
\left[\mathrm{G}_{i}, \mathrm{G}_{j}\right]=f_{i j}{ }^{k} \mathrm{G}_{k} \quad\left\langle\mathrm{G}_{i}, \mathrm{G}_{j}\right\rangle=\Omega_{i j}^{\mathfrak{g}} \quad f_{i j}{ }^{k} \Omega_{k l}^{\mathfrak{g}}+f_{i l}{ }^{k} \Omega_{k j}^{\mathfrak{g}}=0
$$

where $\Omega_{i j}^{\mathfrak{g}}$ denotes the nondegenerate symmetric matrix of metric elements and $f_{i j}{ }^{k}$ the structure constants of the algebra.

Let $\mathfrak{h}$ be a Lie algebra with generators $\mathrm{H}_{\alpha}$ and structure constants $f_{\alpha \beta}^{\gamma}$ that act on $\left(\mathfrak{g},\langle,\rangle_{\mathfrak{g}}\right)$ via antisymmetric derivations, i.e., we have the relations

$$
\left[\mathrm{H}_{\alpha}, \mathrm{G}_{i}\right]=f_{\alpha i}{ }^{j} \mathrm{G}_{j}
$$

and

$$
f_{\alpha i}{ }^{k} \Omega_{k j}^{\mathfrak{g}}+f_{\alpha j}{ }^{k} \Omega_{k i}^{\mathfrak{g}}=0 .
$$

Let $\mathfrak{h}^{*}$ denote the dual of $\mathfrak{h}$. Then the Lie algebra $\mathfrak{d}=D(\mathfrak{g}, \mathfrak{h})$ defined on the vector space direct sum $\mathfrak{g} \dot{+} \mathfrak{h} \dot{+} \mathfrak{h}^{*}$ (spanned by $\mathrm{G}_{i}, \mathrm{H}_{\alpha}$ and $\mathrm{H}^{* \alpha}$, respectively) by

$$
\begin{aligned}
{\left[\mathrm{G}_{i}, \mathrm{G}_{j}\right] } & =f_{i j}{ }^{k} \mathrm{G}_{k}+f_{\alpha i}{ }^{k} \Omega_{k j}^{\mathfrak{g}} \mathrm{H}^{* \alpha} \\
{\left[\mathrm{H}_{\alpha}, \mathrm{G}_{i}\right] } & =f_{\alpha i}{ }^{j} \mathrm{G}_{j} \\
{\left[\mathrm{H}_{\alpha}, \mathrm{H}_{\beta}\right] } & =f_{\alpha \beta}{ }^{\gamma} \mathrm{H}_{\gamma} \\
{\left[\mathrm{H}_{\alpha}, \mathrm{H}^{* \beta}\right] } & =-f_{\alpha \gamma}{ }^{\beta} \mathrm{H}^{* \gamma} \\
{\left[\mathrm{H}^{* \alpha}, \mathrm{G}_{j}\right] } & =0 \\
{\left[\mathrm{H}^{* \alpha}, \mathrm{H}^{* \beta}\right] } & =0
\end{aligned}
$$

is called a double extension of $\mathfrak{g}$ by $\mathfrak{h}$. It permits the invariant metric

$$
\Omega_{a b}^{\mathfrak{d}}=\begin{aligned}
& \mathrm{G}_{i} \\
& \mathrm{H}_{\alpha} \\
& \mathrm{H}^{* \alpha}
\end{aligned}\left(\begin{array}{ccc}
\mathrm{G}_{j} & \mathrm{H}_{\beta} & \mathrm{H}^{* \beta} \\
0 & 0 & 0 \\
0 & h_{\alpha \beta} & \delta_{\alpha}{ }^{\beta} \\
0 & \delta^{\alpha} & 0
\end{array}\right)
$$

where $h_{\alpha \beta}$ is some arbitrary (possibly degenerate or zero) invariant symmetric bilinear form on $\mathfrak{h}$.

Thus, double extensions provide another class of Lie algebras with nondegenerate metric, in addition to semisimple and abelian ones. In a sense to be made more precise below, these classes are the building blocks for all Lie algebras with nondegenerate metric.

Given two Lie algebras with invariant metric $\left(\mathfrak{g}_{1},\langle,\rangle_{1}\right)$ and $\left(\mathfrak{g}_{2},\langle,\rangle_{2}\right)$ a new Lie algebra with invariant metric can be obtained by taking their direct sums and the orthogonal direct product metric

$$
\left(\mathfrak{g}_{1} \oplus \mathfrak{g}_{2},\langle,\rangle_{1} \dot{+}\langle,\rangle_{2}\right) \text {. }
$$


A Lie algebra that can be written in this way is called decomposable otherwise indecomposable. An example of a decomposable Lie algebra is the ads algebra $\mathfrak{s o}(2,2)=$ $\mathfrak{s l}(2, \mathbb{R}) \oplus \mathfrak{s l}(2, \mathbb{R})$.

The proof that all metric Lie algebras can be obtained by direct sums and double extensions of simple and one-dimensional Lie algebras is due to the following structure theorem of Medina and Revoy [42] (with refinements due to [43]).

Theorem 1. Every indecomposable Lie algebra which permits an invariant metric is either:

1. A simple Lie algebra.

2. A one-dimensional Lie algebra.

3. A double extended Lie algebra $D(\mathfrak{g}, \mathfrak{h})$ where:

(a) $\mathfrak{g}$ has no factor $\mathfrak{p}$ for which $H^{1}(\mathfrak{p}, \mathbb{R})=H^{2}(\mathfrak{p}, \mathbb{R})=0$.

(b) $\mathfrak{h}$ is either simple or one-dimensional.

(c) $\mathfrak{h}$ acts on $\mathfrak{g}$ via outer derivations.

Since every decomposable Lie algebra can be obtained from the indecomposable ones this theorem describes how all of them can be generated.

In the following we will use the double extension structure in a more constructive way to answer the question: given a Lie algebra without invariant metric is it possible to add new generators, largely preserving the commutation relations, such that the resulting new algebra has an invariant metric? The double extension structure yields a method to bootstrap a new algebra out of the original one. Suppose the generators of the original algebra can be grouped in two subalgebras $\mathfrak{g}, \mathfrak{h}$ with commutation relations

$$
[\mathfrak{g}, \mathfrak{g}] \subset \mathfrak{g} \quad[\mathfrak{h}, \mathfrak{g}] \subset \mathfrak{g} \quad[\mathfrak{h}, \mathfrak{h}] \subset \mathfrak{h}
$$

such that $\mathfrak{g}$ has a metric $\Omega^{\mathfrak{g}}$ invariant under the action of both $\mathfrak{g}$ and $\mathfrak{h}$. Using the double extension structure we can then construct a new algebra with nondegenerate metric by adding new generators $\mathrm{H}^{\alpha} \in \mathfrak{h}^{*}$. We will thus add $\operatorname{dim}(\mathfrak{h})$ additional generators. Notice that these additional generators will be central extensions for the original algebra if $\mathfrak{h}$ is abelian.

In general, there will exist several choices to group the generators of the original algebra into $\mathfrak{g}$ and $\mathfrak{h}$. A choice that is always possible is to take $\mathfrak{g}$ the trivial algebra and let $\mathfrak{h}$ contain all the generators. The resulting double extension, that is twice as large as the original algebra, is called coadjoint algebra. However, we will usually be interested in the case where $\mathfrak{h}$ has the smallest dimension. In the next sections we will see this procedure at work for the kinematical algebras of section 3 .

Let us note already here that the extensions arrived at using the procedure outlined above will be a mild extension of the original kinematical algebra in the following sense: although in general the new generators $\mathrm{H}^{\alpha}$ are not central they always form an ideal of the resulting extended algebra; cf. equations (4.4d)-(4.4f). Since our definition of homogeneous 
spacetime requires the action of all generators to be effective (cf. appendix B), one should quotient by this ideal when determining the Klein pair of the spacetime. The double extension procedure therefore does not change the underlying homogeneous spacetime but adds only additional generators, such that the metric is well-defined. In the following, we will tacitly assume that a corresponding number of additional fields is included in the CS action.

The interpretation for these additional fields will depend on the details of the model, which we will therefore leave for future work. Let us nevertheless add some general comments. Even though, as we just argued, our changes are rather mild, we want to emphasize that, due to the different field content, different boundary conditions might be required for different extensions. These change the physics, for example in nonrelativistic setups by adding a central element related to mass. Since boundary conditions should reflect the physical solution space, which might differ drastically for different theories (see, e.g, asymptotically flat, dS or AdS boundary conditions) or applications, general statements beyond required consistency requirements are hard to come by.

\section{2 (A)dS, Poincaré, and carrollian algebras}

Let us now investigate how the metric Lie algebras of section 3 that underlie the kinematical gravitational theories can be understood as double extensions.

For the (semi)simple $(\mathfrak{a}) \mathfrak{d} \mathfrak{s}$ algebras there is not much to say, except that they are naturally equipped with an invariant metric given by the Killing form and can be seen as a trivial double extension $D((\mathfrak{a}) \mathfrak{d} \mathfrak{s}, 0)$. The fact that their invariant metric is given by a two parameter family (see $(2.5)$ for $\Lambda \neq 0$ ) is due to the fact that the Lie algebra splits over the complex numbers.

Since the Poincaré algebra in three dimensions is neither semisimple nor abelian but permits an invariant metric the above mentioned theorem guarantees that it is a double extension. In order to see this from the definition of section 4.1, take $\mathfrak{g}$ to be the trivial algebra and $\mathfrak{h}$ to be spanned by $\hat{\mathrm{J}}_{\mu}$ and $\mathfrak{h}^{*}$ by $\hat{\mathrm{P}}^{\mu}$. The commutation relations (4.4c), (4.4d) and (4.4f) reproduce the ones of (2.3) with $\Lambda=0$ when indices are raised and lowered with $\eta_{\mu \nu}$. Thus, we have $\mathfrak{p o i}=D\left(0,\left\{\hat{\mathrm{J}}_{\mu}\right\}\right)$, i.e., it is the coadjoint extension of the Lorentz algebra $\mathfrak{s o}(2,1)$. It is crucial for this to work that there are as many rotations and boosts as there are time and spatial translations. This, being the real magic of three dimensions, is not the case in higher dimensions.

Since AdS-Carroll is isomorphic to the Poincaré algebra upon exchange of $\mathrm{B}_{a}$ and $\mathrm{P}_{a}$ what we just said easily generalizes to this case, as well as to the dS Carroll case.

For the Carroll case we take again $\mathfrak{g}$ as the trivial algebra and consider $\mathfrak{h}$ to be generated by rotation $\mathrm{J}$ and boosts $\mathrm{B}_{a}$. Introducing the generators $\mathrm{P}_{a}=\mathrm{B}_{a}^{*}$ and $\mathrm{H}=\mathrm{J}^{*}$ one reproduces the Carroll algebra. The Carroll algebra is symmetric under the exchange of boosts and spatial momenta. Thus, one can equally well consider it as the double extension of the trivial algebra with $\mathfrak{h}$ generated by $\mathrm{J}$ and $\mathrm{P}$.

\subsection{Double extended (A)dS-Galilei and Galilei}

Let us now turn to the nonrelativistic algebras. This will provide us with the first nontrivial example of the double extension bootstrap which we will treat rather explicitly. 
By our recipe outlined at the end of section 4.1 we should group the generators of the galilean algebras into two subalgebras $\mathfrak{g}, \mathfrak{h}$ compatible with the double extension structure. Looking at the suggestively ordered three dimensional (A)dS Galilei algebras in

$$
\begin{array}{ll}
{\left[\mathrm{J}, \mathrm{B}_{a}\right]=\epsilon_{a b} \mathrm{~B}_{b}} & {\left[\mathrm{~J}, \mathrm{P}_{a}\right]=\epsilon_{a b} \mathrm{P}_{b}} \\
{\left[\mathrm{H}, \mathrm{B}_{a}\right]=\epsilon_{a b} \mathrm{P}_{b}} & {\left[\mathrm{H}, \mathrm{P}_{a}\right]= \pm \Lambda^{2} \epsilon_{a b} \mathrm{~B}_{b}}
\end{array}
$$

one recognizes that $\{\mathrm{J}, \mathrm{H}\}$ acts on $\left\{\mathrm{B}_{a}, \mathrm{P}_{a}\right\}$ as antisymmetric derivations. Since the commutators of $\left\{\mathrm{B}_{a}, \mathrm{P}_{a}\right\}$ are abelian we can define an invariant metric $\Omega_{k j}^{\mathfrak{g}}$ on this subalgebra and identify it as $\mathfrak{g} \simeq\left\{\mathrm{B}_{a}, \mathrm{P}_{a}\right\}$ which implies $f_{i j}{ }^{k}=0$ in (4.4a). The action of $\mathfrak{h}=\{\mathrm{J}, \mathrm{H}\}$ on $\mathfrak{g}$ fixes $f_{\alpha i}{ }^{j}$ in (4.4b), and the commutation relations in equation (4.8) show that $f_{\alpha \beta}{ }^{\gamma}=0$ in (4.4c). The elements of $\mathfrak{h}^{*}$ will therefore play the rôle of central extensions of the original algebra.

In order for this choice of $\mathfrak{g}, \Omega_{k j}^{\mathfrak{g}}$, and $\mathfrak{h}$ to be admissible for a double extension one has to check that condition (4.3) is satisfied. Invariance under the action of J restricts $\Omega_{k j}^{\mathfrak{g}}$ to

$$
\left\langle\mathrm{P}_{a}, \mathrm{P}_{b}\right\rangle=\mu_{\mathrm{P}} \delta_{a b} \quad\left\langle\mathrm{~B}_{a}, \mathrm{~B}_{b}\right\rangle=\mu_{\mathrm{B}} \delta_{a b} \quad\left\langle\mathrm{~B}_{a}, \mathrm{P}_{b}\right\rangle=\mu_{\mathrm{BP}} \delta_{a b}+\tilde{\mu}_{\mathrm{BP}} \epsilon_{a b} .
$$

From invariance under the action of $\mathrm{H}$ we find the conditions

$$
\tilde{\mu}_{\mathrm{BP}}=0 \quad \mu_{\mathrm{P}}= \pm \Lambda^{2} \mu_{\mathrm{B}} .
$$

Note that there exist two more options for grouping the generators into $\mathfrak{g}$ and $\mathfrak{h}$ according to (4.7). The choice $\mathfrak{g}=\left\{\mathrm{H}, \mathrm{P}_{a}, \mathrm{~B}_{a}\right\}$ would result in a smaller number of additional generators, however one cannot define a nondegenerate metric $\Omega^{\mathfrak{g}}$ on $\mathfrak{g}$, thus excluding this case. The other choice $\mathfrak{g}=\{0\}$ corresponds to the coadjoint extension that is possible for any algebra but leads to a doubling of the algebra. The case $\mathfrak{g}=\left\{\mathrm{B}_{a}, \mathrm{P}_{a}\right\}$ is therefore preferred due to the smaller number of additional generators.

Based on the two-parameter family $\left(\mu_{\mathrm{BP}}, \mu_{\mathrm{B}}\right)$ we can now start the double extension procedure. Since the algebra $\mathfrak{h}$ generated by $\mathrm{H}$ and $\mathrm{J}$ is again an abelian algebra any two-dimensional metric will be invariant. We choose the metric to be of the form

$$
\langle\mathrm{H}, \mathrm{J}\rangle=-\mu \quad\langle\mathrm{J}, \mathrm{J}\rangle=-\chi \quad\langle\mathrm{H}, \mathrm{H}\rangle=\chi_{\mathrm{H}} .
$$

The dual elements that we denote by $\mathfrak{h}^{*}=\left\{\mathrm{J}^{*}, \mathrm{H}^{*}\right\}$ have the metric

$$
\left\langle\mathrm{J}, \mathrm{J}^{*}\right\rangle=1 \quad\left\langle\mathrm{H}, \mathrm{H}^{*}\right\rangle=1 .
$$

The double extension structure now dictates via equation (4.4a) the following change in the commutation relations of $\mathfrak{g}$ :

$$
\begin{aligned}
& {\left[\mathrm{B}_{a}, \mathrm{P}_{b}\right]=\epsilon_{a b}\left(\mu_{\mathrm{BP}} \mathrm{J}^{*} \pm \Lambda^{2} \mu_{\mathrm{B}} \mathrm{H}^{*}\right) \quad\left[\mathrm{B}_{a}, \mathrm{~B}_{b}\right]=\epsilon_{a b}\left(\mu_{\mathrm{B}} \mathrm{J}^{*}+\mu_{\mathrm{BP}} \mathrm{H}^{*}\right)} \\
& {\left[\mathrm{P}_{a}, \mathrm{P}_{b}\right]= \pm \Lambda^{2} \epsilon_{a b}\left(\mu_{\mathrm{B}} \mathrm{J}^{*}+\mu_{\mathrm{BP}} \mathrm{H}^{*}\right) .}
\end{aligned}
$$

From comparison with the metric of the galilean algebras that we obtained in section 3.3 we find that we should identify

$$
\mu_{\mathrm{BP}}=\alpha^{2} \mu_{\mathrm{MS}} \quad \mu_{\mathrm{B}}=\alpha^{2} \mu_{\mathrm{S}} .
$$


Setting now

$$
\alpha \mathrm{M}=\mu_{\mathrm{BP}} \mathrm{J}^{*} \pm \Lambda^{2} \mu_{\mathrm{B}} \mathrm{H}^{*} \quad \alpha \mathrm{S}=\mu_{\mathrm{B}} \mathrm{J}^{*}+\mu_{\mathrm{BP}} \mathrm{H}^{*}
$$

in (4.13) and (4.12), we reproduce together with (4.8) and (4.11) precisely the commutation relations and metric elements in table 2 of the galilean theories. The freedom in choosing $\chi_{\mathrm{H}}$ in (4.11) corresponds to the term in (3.13) that is an additional freedom in the choice of invariant bilinear form for the galilean theories not apparent from table 2 .

\section{Non-contracting theories}

In the last section we were concerned with kinematical Lie algebras that arise as contractions of the simple $(\mathfrak{a}) \mathfrak{d} \mathfrak{s}$ algebra. These kinematical algebras are well-known since the seminal work of Bacry and Lévy-Leblond [38]. However, there exist kinematical algebras that do not arise as a limit but nevertheless lead to well-defined kinematical spacetimes. These have been classified only recently [40], and some of them are based on kinematical Lie algebras that are special to $2+1$ dimensions [55]. In this section we want to study if it is possible to construct Chern-Simons theories based on these algebras, i.e., if these algebras, or any of their possible central extensions, allow for a nondegenerate invariant metric. If the centrally extended algebras do not allow for an invariant metric, we will examine whether there exists a double extension based on these algebras with an invariant metric by construction. In case double extensions with fewer additional generators do not exist the coadjoint extension is always possible. Applying these three tools to construct metric extensions of a given algebra we analyze the remaining three-dimensional kinematical algebras in the following.

\subsection{Torsional galilean theories}

As our first class of non-contracting kinematic Lie algebras we treat the so-called torsional galilean theories. All of them share the commutators

$$
\left[\mathrm{J}, \mathrm{B}_{a}\right]=\epsilon_{a b} \mathrm{~B}_{b} \quad\left[\mathrm{~J}, \mathrm{P}_{a}\right]=\epsilon_{a b} \mathrm{P}_{b} \quad\left[\mathrm{H}, \mathrm{B}_{a}\right]=\epsilon_{a b} \mathrm{P}_{b} .
$$

For the torsional galilean-dS algebra we add

$$
\left[\mathrm{H}, \mathrm{P}_{a}\right]=\gamma \epsilon_{a b} \mathrm{~B}_{b}+(1+\gamma) \mathrm{P}_{a},
$$

while for the torsional galilean-AdS algebra we add

$$
\left[\mathrm{H}, \mathrm{P}_{a}\right]=\left(1+\chi^{2}\right) \epsilon_{a b} \mathrm{~B}_{b}+2 \chi \mathrm{P}_{a} .
$$

The free parameters are constrained by $\gamma \in[-1,1), \chi>0$. For $\chi=0$, the interpolating algebra reduces to galilean AdS, while $\gamma=-1$ corresponds to galilean dS. Let us emphasize that the spacetimes associated to these algebras differ for different $\gamma$ and $\chi$ which means that these parameters should not be understood as contraction parameters. The algebras (5.2) and (5.3) exhibit the same invariant structure common to all galilean theories given by invariant one-form and invariant co-metric. Different from the usual (A)dSgalilean or flat galilean algebras, the invariant connection associated to the homogeneous spacetimes of (5.2) and (5.3) exhibits torsion, hence their name [40]. 
While these torsional galilean algebras exist in any dimension, in $2+1$ dimensions one has an additional two-parameter family interpolating between the two of them, labelled S12 in the classification of [40], which is given by (5.1) and

$$
\left[\mathrm{H}, \mathrm{P}_{a}\right]=(1+\gamma) \mathrm{P}_{a}-\chi \epsilon_{a b} \mathrm{P}_{b}+\gamma \epsilon_{a b} \mathrm{~B}_{b}+\chi \mathrm{B}_{a},
$$

where the parameters $\gamma, \chi$ are restricted to the same range as above.

All torsional galilean Lie algebras have a unique central extension given by

$$
[\mathrm{J}, \mathrm{H}]=\mathrm{Z} .
$$

Applying the necessary criterion of (3.6) to the torsional galilean algebras we see that none of them comes equipped with a nondegenerate invariant metric, regardless of the presence or absence of the central charge. Indeed, they all share, irrespective of the presence of the central extension (5.5), the same most general degenerate invariant metric given by

$$
\langle\mathrm{J}, \mathrm{J}\rangle=\chi_{\mathrm{J}} \quad\langle\mathrm{J}, \mathrm{H}\rangle=\chi_{\mathrm{JH}} \quad\langle\mathrm{H}, \mathrm{H}\rangle=\chi_{\mathrm{H}} .
$$

Thus, it is not possible to have well-defined Chern-Simons theories based on the above (centrally extended) algebras.

Let us therefore turn to double extensions. We will proceed as we did for the galilean algebras in section 4.3 by splitting the algebra into subalgebras $\mathfrak{g}$ and $\mathfrak{h}$, adding new generators corresponding to the dual of $\mathfrak{h}$. In particular, we want to find the double extension with the smallest number of additional generators.

We treat the cases of torsional galilean (A)dS algebra and the interpolating algebra at once by writing the second commutator of equations (5.2), (5.3), and (5.4), respectively, as

$$
\left[\mathrm{H}, \mathrm{P}_{a}\right]=\alpha \epsilon_{a b} \mathrm{~B}_{b}+\beta \mathrm{P}_{a}+\tilde{\beta} \epsilon_{a b} \mathrm{P}_{b}+\tilde{\alpha} \mathrm{B}_{a},
$$

with constant $\alpha, \beta, \tilde{\alpha}, \tilde{\beta}$ according to the respective algebra.

In order to bootstrap a new double extended algebra out of this we have to choose subalgebras $\mathfrak{g}$ and $\mathfrak{h}$. The only choice, apart from the coadjoint extension, compatible with the double extension structure is $\mathfrak{g}=\left\{\mathrm{G}_{a}, \mathrm{P}_{a}\right\}$ and $\mathfrak{h}=\{\mathrm{J}, \mathrm{H}\}$. However, this choice of $\mathfrak{g}$ and $\mathfrak{h}$ is not compatible with a nondegenerate metric. Since $\mathfrak{h}$ is abelian, the new generators appearing through $\mathfrak{h}^{*}$ are necessarily central. Yet, we discussed already in section 5.1 that adding central extensions is not enough to get a nondegenerate metric. Thus, the only option left is to take $\mathfrak{h}$ to contain all the generators so that we end up with the coadjoint algebra of the torsional galilean theories. More explicitly, we introduce the elements $\left\{\mathrm{H}^{*}, \mathrm{~J}^{*}, \mathrm{~B}_{a}^{*}, \mathrm{P}_{a}^{*}\right\}$ together with the brackets summarized on the left hand side of table 3 , which leads to the coadjoint metric on the right hand side.

\subsection{Carrollian light cone}

Among the non-contracting kinematical Lie algebras the carrollian light cone algebra appears to be the most elusive one. Its commutation relations are given, in addition to the usual brackets $\left[\mathrm{J}, \mathrm{B}_{a}\right]=\epsilon_{a b} \mathrm{~B}_{b}$ and $\left[\mathrm{J}, \mathrm{P}_{a}\right]=\epsilon_{a b} \mathrm{P}_{b}$, by

$$
\left[\mathrm{B}_{a}, \mathrm{P}_{b}\right]=-\mathrm{H} \epsilon_{a b}-\mathrm{J} \delta_{a b} \quad\left[\mathrm{H}, \mathrm{P}_{a}\right]=-\mathrm{P}_{a} \quad\left[\mathrm{H}, \mathrm{B}_{a}\right]=\mathrm{B}_{a} .
$$




\begin{tabular}{|lr|}
\hline & torsional galilean \\
\hline$\left[\mathrm{J}, \mathrm{X}_{a}^{*}\right]$ & $\epsilon_{a b} \mathrm{X}_{b}^{*}$ \\
{$\left[\mathrm{H}, \mathrm{B}_{a}^{*}\right]$} & $-\tilde{\alpha} \mathrm{P}_{a}^{*}+\alpha \epsilon_{a b} \mathrm{P}_{b}^{*}$ \\
{$\left[\mathrm{H}, \mathrm{P}_{a}^{*}\right]$} & $\epsilon_{a b} \mathrm{~B}_{b}^{*}-\beta \mathrm{P}_{a}^{*}+\tilde{\beta} \epsilon_{a b} \mathrm{P}_{b}^{*}$ \\
{$\left[\mathrm{~B}_{a}, \mathrm{~B}_{b}^{*}\right]$} & $\epsilon_{a b} \mathrm{~J}^{*}$ \\
{$\left[\mathrm{P}_{a}, \mathrm{P}_{b}^{*}\right]$} & $\epsilon_{a b} \mathrm{~J}^{*}+\beta \delta_{a b} \mathrm{H}^{*}+\tilde{\beta} \epsilon_{a b} \mathrm{H}^{*}$ \\
{$\left[\mathrm{P}_{a}, \mathrm{~B}_{b}^{*}\right]$} & $\alpha \epsilon_{a b} \mathrm{H}^{*}+\tilde{\alpha} \delta_{a b} \mathrm{H}^{*}$ \\
\hline
\end{tabular}

\begin{tabular}{|lr|}
\hline \multicolumn{2}{|l|}{ torsional galilean } \\
\hline$\left\langle\mathrm{J}, \mathrm{J}^{*}\right\rangle$ & $\chi$ \\
$\left\langle\mathrm{H}, \mathrm{H}^{*}\right\rangle$ & $\chi$ \\
$\left\langle\mathrm{B}_{a}, \mathrm{~B}_{b}^{*}\right\rangle$ & $\chi \delta_{a b}$ \\
$\left\langle\mathrm{P}_{a}, \mathrm{P}_{b}^{*}\right\rangle$ & $\chi \delta_{a b}$ \\
$\langle\mathrm{~J}, \mathrm{~J}\rangle$ & $\mu_{\mathrm{J}}$ \\
$\langle\mathrm{J}, \mathrm{H}\rangle$ & $\mu_{\mathrm{J}}$ \\
$\langle\mathrm{H}, \mathrm{H}\rangle$ & $\mu_{\mathrm{H}}$ \\
\hline
\end{tabular}

Table 3. Coadjoint extension of the torsional galilean algebras. $\mathrm{X}_{a}^{*}$ stands for $\mathrm{B}_{a}^{*}, \mathrm{P}_{a}^{*}$. The metric is nondegenerate for $\chi \neq 0$.

Although not obvious from this choice of basis, the carrollian light cone algebra is a simple Lie algebra. In three dimensions it is isomorphic to $\mathfrak{s o}(3,1)$. We conclude that all central extensions are necessarily trivial and that a nondegenerate invariant bilinear form for the carrollian light cone is provided by the Killing form. More explicitly, the most general invariant metric is

$$
\langle\mathrm{J}, \mathrm{J}\rangle=-\langle\mathrm{H}, \mathrm{H}\rangle=\chi \quad\langle\mathrm{J}, \mathrm{H}\rangle=\mu \quad\left\langle\mathrm{B}_{a}, \mathrm{P}_{b}\right\rangle=-\mu \delta_{a b}+\chi \epsilon_{a b},
$$

which becomes degenerate for $\chi=\mu=0$. The two-parameter family of metrics is again due to the fact that $\mathfrak{s o}(3,1)$ splits as a complex Lie algebra. From the fact that both the carrollian light cone algebra and the $\mathfrak{d} \mathfrak{s}$ algebra are isomorphic to $\mathfrak{s o}(3,1)$ one should not conclude that both lead to identical gravitational Chern-Simons theories. The point of view that we aim to stress throughout this work is precisely that the specification of an abstract algebra is not enough to define the gravitational Chern-Simons theory. In particular, note that the subalgebra $\mathfrak{b}=\left\{\mathrm{J}, \mathrm{B}_{a}\right\}$ is different in both cases.

A puzzling feature of the Lie algebra (5.8) is the fact that it is not reductive. This means that it does not have a decomposition as $\mathfrak{g}=\mathfrak{b} \oplus \mathfrak{m}$ such that both subspaces transform as adjoint $\mathfrak{b}$-modules. Acting with a boost thus mixes up the separation of the different subspaces. Some aspects of a Chern-Simons theory based on this kinematical algebra are discussed in [65]. We will have further comments on potential applications in the discussion.

\subsection{Aristotelian}

The remaining cases of kinematical algebras that do not derive from a contraction of AdS belong to the class of aristotelian algebras. An example for members of this class, that we saw earlier, is given by the static algebra (once it is quotiented by the boosts). These aristotelian algebras and their associated spacetimes have been classified only recently [40, table 2] and have therefore evaded a systematic study of their metric properties. They arise due to the fact that the action of the boosts on the spacetime is not effective, which makes it natural to quotient by them. From this point of view, the aforementioned para-Galilei 
spacetime is then equivalent to the aristotelian static spacetime. ${ }^{14}$ Let us also note that all aristotelian spacetimes contract to the aristotelian static one.

All aristotelian Lie algebras are spanned by $\mathrm{J}, \mathrm{H}$ and $\mathrm{P}_{a}$ with only

$$
\left[\mathrm{J}, \mathrm{P}_{a}\right]=\epsilon_{a b} \mathrm{P}_{b}
$$

remaining of the usual kinematic brackets. Before we examine the various options for the remaining commutators let us emphasize that in a CS interpretation the field $\omega$ in the connection $A$ does only have a component along the $\mathrm{J}$ direction.

\subsubsection{Aristotelian static and torsional static}

For the aristotelian static spacetime the only nonzero commutator is given by (5.10). For the torsional static spacetime we need to add the commutator

$$
\left[\mathrm{H}, \mathrm{P}_{a}\right]=\mathrm{P}_{a}
$$

Both permit no nondegenerate invariant metric, as can be checked by criterion (3.6). Their most general symmetric, invariant bilinear form is again given by (5.6).

The unique central extension for the torsional static spacetime is

$$
[\mathrm{J}, \mathrm{H}]=\mathrm{Z}_{\mathrm{JH}} .
$$

For the aristotelian static there is a second central extension given by

$$
\left[\mathrm{P}_{a}, \mathrm{P}_{b}\right]=\epsilon_{a b} \mathrm{Z}_{\mathrm{P}}
$$

The aristotelian static algebra does have a nondegenerate invariant metric if only the central extension $Z_{\mathrm{P}}$ is added. ${ }^{15}$ It is given by (5.6) with the addition of

$$
\left\langle\mathrm{P}_{a}, \mathrm{P}_{b}\right\rangle=\delta_{a b} \chi_{\mathrm{P}} \quad\left\langle\mathrm{Z}_{\mathrm{P}}, \mathrm{Z}_{\mathrm{P}}\right\rangle=\chi_{\mathrm{P}}
$$

under the condition that $\chi_{\mathrm{P}}, \chi_{\mathrm{H}} \neq 0$.

The torsional static spacetime on the other hand does not have an invariant metric even if the central extension $\mathrm{Z}_{\mathrm{JH}}$ is added.

In order to construct a nondegenerate metric for the torsional aristotelian static algebra we therefore turn again to the double extension construction. The only permissible choice of subalgebra $\mathfrak{g}$ compatible with the double extension structure is $\mathfrak{g}=\left\{\mathrm{P}_{a}\right\}$. However, any possible choice of invariant metric $\left\langle\mathrm{P}_{a}, \mathrm{P}_{b}\right\rangle$ is disallowed by the condition

$$
\left\langle\left[\mathrm{H}, \mathrm{P}_{a}\right], \mathrm{P}_{b}\right\rangle+\left\langle\mathrm{P}_{a},\left[\mathrm{H}, \mathrm{P}_{b}\right]\right\rangle=0
$$

\footnotetext{
${ }^{14}$ This construction does not necessarily generalize once the invariant metric is also taken into account, as we saw, e.g., for the static theory in section 3.3. To distinguish theories based on the static spacetime with boosts from the ones without, we have added the specification "aristotelian" to the latter.

${ }^{15}$ This can be understood as a double extension with respect to $\mathrm{J}$.
} 
Thus again, the only viable double extension is the coadjoint extension given explicitly by

$$
\begin{aligned}
{\left[\mathrm{J}, \mathrm{P}_{a}\right] } & =\epsilon_{a b} \mathrm{P}_{b} \quad\left[\mathrm{H}, \mathrm{P}_{a}\right]=\mathrm{P}_{a} \\
{\left[\mathrm{~J}, \mathrm{P}_{a}^{*}\right] } & =\epsilon_{a b} \mathrm{P}_{b}^{*} \quad\left[\mathrm{H}, \mathrm{P}_{a}^{*}\right]=-\mathrm{P}_{a}^{*} \\
{\left[\mathrm{P}_{a}, \mathrm{P}_{b}^{*}\right] } & =\epsilon_{a b} \mathrm{~J}^{*}+\delta_{a b} \mathrm{H}^{*} .
\end{aligned}
$$

The most general invariant metric consists of a part given by (5.6) (which is irrelevant for the nondegeneracy), the usual coadjoint invariant metric $\left\langle\mathrm{x}_{a}, \mathrm{x}_{b}^{*}\right\rangle=\mu \delta_{a b}(\mu \neq 0)$ for all elements of the original algebra $\mathrm{X}_{a}$ and their dual $\mathrm{X}_{b}^{*}$, and a second invariant metric $(\chi \neq 0)$ given by

$$
\left\langle\mathrm{H}, \mathrm{J}^{*}\right\rangle=\chi \quad\left\langle\mathrm{J}, \mathrm{H}^{*}\right\rangle=-\chi \quad\left\langle\mathrm{P}_{a}, \mathrm{P}_{b}^{*}\right\rangle=\epsilon_{a b} \chi .
$$

\subsection{2 $\mathbb{R} \times \mathbb{H}^{2}$ and $\mathbb{R} \times \mathbb{S}^{2}$}

Besides (5.10) the other nonzero commutators for $\mathbb{R} \times \mathbb{H}^{2}$ (minus sign) and $\mathbb{R} \times \mathbb{S}^{2}$ (plus sign) are given by

$$
\left[\mathrm{P}_{a}, \mathrm{P}_{b}\right]= \pm \epsilon_{a b} \mathrm{~J}
$$

We note in passing that the case $\mathbb{R} \times \mathbb{S}^{2}$ corresponds to the Einstein static universe in $2+1$ dimensions.

Since they are a direct sum of a simple and abelian factor it can be anticipated that they do not posses nontrivial central extensions. From the above follows that one can construct an invariant metric given by

$$
\langle\mathrm{J}, \mathrm{J}\rangle=\chi \quad\left\langle\mathrm{P}_{a}, \mathrm{P}_{b}\right\rangle=\mp \chi \delta_{a b} \quad\langle\mathrm{H}, \mathrm{H}\rangle=\chi_{\mathrm{H}}
$$

which is nondegenerate for $\chi, \chi_{\mathrm{H}} \neq 0$.

\subsubsection{A24}

This spacetime is special to $2+1$ dimensions as can be seen by the commutator

$$
\left[\mathrm{P}_{a}, \mathrm{P}_{b}\right]=\epsilon_{a b} \mathrm{H}
$$

which has no higher dimensional analog. It does not permit central extensions, but has an invariant metric given by

$$
\langle\mathrm{P}, \mathrm{P}\rangle=\mp \chi \delta_{a b} \quad\langle\mathrm{~J}, \mathrm{H}\rangle=\chi \quad\langle\mathrm{J}, \mathrm{J}\rangle=\chi_{\mathrm{J}} .
$$

which is nondegenerate for $\chi \neq 0$.

\section{Generalization to higher dimensions}

Until this point our discussion was centered around the necessary data to define ChernSimons theories based on the kinematical spacetimes of $[38,40]$. The double extension 
structure turned out to be a useful tool in this endeavor as it allows us to construct metric Lie algebras as (not necessarily central) extensions of a given kinematical Lie algebra.

In this section we want to push this program to higher dimensions, i.e., given a kinematical Lie algebra without nondegenerate metric we aim to construct a double extension using the smallest number of additional generators. We will apply this program again to all kinematical Lie algebras associated to the homogeneous spacetimes of [40].

While extensions of some of these Lie algebras have already been studied in the literature [66] we analyze it with emphasis towards invariant metrics and double extensions. Since the Lie algebras considered in theories of three-dimensional gravity, at least in the Chern-Simons formulation, are necessarily metric Lie algebras our construction can be regarded as a starting point for a natural generalization of the gauge theory formulation of three-dimensional gravity to higher dimensions.

Since we work in generic dimensions we employ a somewhat different notation summarized in appendix A. In particular, the dualization of angular momentum and boosts by the three-dimensional epsilon tensor is not implied anymore and the Greek indices $\mu, \nu, \ldots$ run over space and time.

Finally let us emphasize that even though we will refer in the following to the Lie algebras, we are interested in their interpretation as spacetimes. Isomorphic Lie algebras differ due to different choices of associated Klein pairs.

\section{1 (Anti-)de Sitter algebras}

The (A)dS algebra in $D+1$ dimensions is given in table 4. We refer to appendix A for further details on the rescaling and space-time split of the (A)dS algebra. The generator $\mathrm{M}$ is the single element of the trivial central extension $\mathfrak{u}(1)$ of the simple $(\mathrm{A}) \mathrm{dS}$ algebra that can be reabsorbed in the algebra by a shift of $\mathrm{H}$. However, we have included this central extension for later use in section 7 . There we will find that, as in the three-dimensional case presented in section 3, the central extension will cease to be trivial in the nonrelativistic limit. More precisely, after the nonrelativistic limit the resulting algebra is the Bargmann algebra.

For generic dimension the simple (anti-)de Sitter algebras still permit an invariant metric which is proportional to the Killing form. Since the split of the algebra to a semisimple (complex) one is special to $2+1$ dimensions the invariant metric in generic dimension is parametrized by the single constant $\chi$ (and an arbitrary choice of normalization for $\mathrm{M}$ given by $\chi_{\mathrm{M}}$ ). Since the $(\mathrm{A}) \mathrm{dS}$ algebra already has an invariant metric in any dimension we do not employ the double extension bootstrap.

\subsection{Metric Poincaré algebra and the Maxwell algebra}

As was mentioned at several points already, the Poincaré algebra in arbitrary dimensions does not permit an invariant bilinear metric. Yet it is possible to add new generators to the algebra while preserving most of its commutation relations such that the resulting algebra has an invariant metric.

The Poincare algebra is contained in table 4 in the $\Lambda \rightarrow 0$ limit setting all other contraction parameters to unity and quotienting by $\mathrm{M}$. 


\begin{tabular}{|c|c|c|c|}
\hline \multicolumn{2}{|c|}{$[(\mathfrak{a}) \mathfrak{d} \mathfrak{s} \oplus \mathfrak{u}(1)]_{c_{i}, \tau, \Lambda}$} & \multirow{2}{*}{\multicolumn{2}{|c|}{$[(\mathfrak{a}) \mathfrak{d} \mathfrak{s} \oplus \mathfrak{u}(1)]_{c_{i}, \tau, \Lambda}$}} \\
\hline \multirow{7}{*}{$\begin{array}{l}{\left[\mathrm{J}_{a b}, \mathrm{~J}_{c d}\right]} \\
{\left[\mathrm{J}_{a b}, \mathrm{~B}_{c}\right]} \\
{\left[\mathrm{J}_{a b}, \mathrm{P}_{c}\right]} \\
{\left[\mathrm{B}_{a}, \mathrm{~B}_{b}\right]} \\
{\left[\mathrm{B}_{a}, \mathrm{P}_{b}\right]} \\
{\left[\mathrm{B}_{a}, \mathrm{H}\right]} \\
{\left[\mathrm{P}_{a}, \mathrm{H}\right]} \\
{\left[\mathrm{P}_{a}, \mathrm{P}_{b}\right]}\end{array}$} & \multirow{7}{*}{$\begin{array}{r}4 \delta_{[a \mid[c} \mathrm{J}_{d] \mid b]} \\
-2 \delta_{c[a} \mathrm{B}_{b]} \\
-2 \delta_{c[a} \mathrm{P}_{b]} \\
\tau^{2} c_{i}^{2} \mathrm{~J}_{a b} \\
c_{i}^{2} \delta_{a b} \mathrm{H}+\alpha \delta_{a b} \mathrm{M} \\
\tau^{2} \mathrm{P}_{a} \\
\mp \Lambda^{2} \mathrm{~B}_{a} \\
\pm c_{i}^{2} \Lambda^{2} \mathrm{~J}_{a b}\end{array}$} & & \\
\hline & & $\left\langle\mathrm{J}_{a b}, \mathrm{~J}_{c d}\right\rangle$ & $2 \chi \delta_{a[c} \delta_{d] b}$ \\
\hline & & $\left\langle\mathrm{B}_{a}, \mathrm{~B}_{b}\right\rangle$ & $-\chi \tau^{2} c_{i}^{2} \delta_{a b}$ \\
\hline & & $\langle\mathrm{H}, \mathrm{H}\rangle$ & $\tau^{2} \Lambda^{2}\left( \pm \chi+\alpha^{2} \chi_{\mathrm{M}}\right)$ \\
\hline & & $\left\langle\mathrm{P}_{a}, \mathrm{P}_{b}\right\rangle$ & $\mp \chi c_{i}^{2} \Lambda^{2} \delta_{a b}$ \\
\hline & & $\langle\mathrm{M}, \mathrm{M}\rangle$ & $\left(\Lambda \tau c_{i}^{2}\right)^{2} \chi_{\mathrm{M}}$ \\
\hline & & & \\
\hline
\end{tabular}

Table 4. Commutation relations and invariant metric for the trivially centrally extended $\mathfrak{a} \mathfrak{d} \mathfrak{s}$ (upper sign) and $\mathfrak{d s}$ (lower sign) algebras in dimension $D+1$.

\begin{tabular}{|lr|}
\hline & Maxwell \\
\hline$\left[\mathrm{P}_{\mu}, \mathrm{P}_{\nu}\right]$ & $-\chi_{\mathrm{P}} \mathrm{M}_{\mu \nu}^{*}$ \\
{$\left[\mathrm{M}_{\mu \nu}, \mathrm{P}_{\rho}\right]$} & $-2 \eta_{\rho[\mu} \mathrm{P}_{\nu]}$ \\
{$\left[\mathrm{M}_{\mu \nu}, \mathrm{M}_{\rho \lambda}\right]$} & $4 \eta_{\left[\mu \mid\left[\rho \mathrm{M}_{\lambda] \mid \nu]}\right.\right.}$ \\
{$\left[\mathrm{M}_{\mu \nu}, \mathrm{M}_{\rho \lambda}^{*}\right]$} & $4 \eta_{[\mu \mid[\rho} \mathrm{M}_{\lambda] \mid \nu]}^{*}$ \\
\hline
\end{tabular}

\begin{tabular}{|rr|}
\hline & Maxwell \\
\hline$\left\langle\mathrm{P}_{\mu}, \mathrm{P}_{\nu}\right\rangle$ & $\chi_{\mathrm{P}} \eta_{\mu \nu}$ \\
$\left\langle\mathrm{M}_{\mu \nu}, \mathrm{M}_{\rho \lambda}^{*}\right\rangle$ & $2 \eta_{\mu[\rho} \eta_{\lambda] \nu}$ \\
$\left\langle\mathrm{M}_{\mu \nu}, \mathrm{M}_{\rho \lambda}\right\rangle$ & $2 \chi_{\mathrm{M}} \eta_{\mu[\rho} \eta_{\lambda] \nu}$ \\
\hline
\end{tabular}

Table 5. The double extension of the Poincaré algebra is the Maxwell algebra. We have lowered the indices of $\mathrm{M}^{* \mu \nu}$ by $\eta_{\mu \nu}$. The invariant metric is nondegenerate for $\chi_{\mathrm{P}} \neq 0$.

Switching back to relativistic notation for the moment, we find that the only choice compatible with the double extension structure is $\mathfrak{g}=\left\{\mathrm{P}_{\mu}\right\}$, and $\mathfrak{h}=\left\{\mathrm{M}_{\mu \nu}\right\}$. The abelian subalgebra generated by $\mathrm{P}_{\mu}$ has a Lorentz-invariant metric given by the Minkowski metric. With these ingredients we can construct a double extension by introducing the generators $\mathfrak{h}^{*}=\left\{\mathrm{M}_{\mu \nu}^{*}\right\}$. The double extension then dictates the commutation relations in table 5 .

Remarkably, the double extension leads us to the so called Maxwell algebra. This long-known algebra describes the symmetries of a particle in a classical homogeneous electromagnetic field $[67,68]$. The existence of an invariant metric for this algebra has been noted in the literature (see, e.g., [69]); here we want to emphasize that the Maxwell algebra is the most natural extension with an invariant metric of the Poincare algebra from the point of view of double extensions.

Our construction also perfectly agrees with Theorem 1 , since we double extend the abelian translations $\mathfrak{g}=\left\{\mathrm{P}_{\mu}\right\}$ (which has no factor $\mathfrak{p}$ for which $H^{1}(\mathfrak{p}, \mathbb{R})$ and $H^{2}(\mathfrak{p}, \mathbb{R})$ are vanishing) by the simple Lorentz algebra $\mathfrak{h}=\left\{\mathrm{M}_{\mu \nu}\right\} \simeq \mathfrak{s o}(D-1,1)$.

\subsection{Metric carrollian algebras}

Let us now turn to the ultrarelativistic algebras whose commutation relations are given by the ultrarelativistic limit, $\tau \rightarrow 0$, of table 4 where we ignore the trivial central extension and set $c_{i}=1$. 
The carrollian algebras do not support a nondegenerate metric in any dimension other than three, see, e.g., [70]. Due to rotational invariance the only nonvanishing metric element of $\mathrm{H}$ is with itself. But the invariance condition implies

$$
0 \stackrel{!}{=} \mathrm{B}_{a} \cdot\left\langle\mathrm{H}, \mathrm{P}_{b}\right\rangle=\left\langle\mathrm{H},\left[\mathrm{B}_{a}, \mathrm{P}_{b}\right]\right\rangle=\delta_{a b}\langle\mathrm{H}, \mathrm{H}\rangle,
$$

which renders the metric degenerate. In order to obtain a nondegenerate metric we will employ again the double extension procedure.

Starting with the flat Carroll-algebra $(\Lambda \rightarrow 0)$ the first step is to determine a subalgebra $\mathfrak{g}$ with nondegenerate metric. Due to the above argument we see that this subalgebra cannot be $\left\{\mathrm{P}_{a}, \mathrm{H}\right\}$. The only other option allowed by the structure of the algebra is $\left\{\mathrm{B}_{a}, \mathrm{H}\right\}$. However, the flat Carroll algebra is symmetric under the exchange of $\mathrm{B}_{a}$ and $\mathrm{P}_{a}$ which excludes the second option, as well. Thus, the Carroll algebra cannot be the starting point for a double extension apart from the coadjoint extension (in which H is taken to lie in $\mathfrak{h}$ ). ${ }^{16}$

Let us turn now to the (A)dS-carrollian algebras. The argument of equation (6.1) still applies, thus $\left\{\mathrm{P}_{a}, \mathrm{H}\right\}$ is no option for the subalgebra $\mathfrak{g}$ of the double extension. Since the (A)dS-Carroll algebra is not symmetric under the exchange of boosts and spatial translations, one can choose $\mathfrak{g}=\left\{\mathrm{B}_{a}, \mathrm{H}\right\}$. A rotational-invariant metric for $\mathfrak{g}$ is given by

$$
\left\langle\mathrm{B}_{a}, \mathrm{~B}_{b}\right\rangle=\chi_{\mathrm{B}} \delta_{a b} \quad\langle\mathrm{H}, \mathrm{H}\rangle=\chi_{\mathrm{H}} .
$$

Invariance under the action of $\mathrm{P}_{a}$ implies the condition

$$
\chi_{\mathrm{H}}=\mp \Lambda^{2} \chi_{\mathrm{B}} .
$$

Using these as our starting point for a double extension process we find, introducing the generators $\mathfrak{h}^{*}=\left\{\mathrm{J}_{a b}^{*}, \mathrm{P}_{a}^{*}\right\}$, the double extension algebra $(\mathfrak{a}) \mathfrak{d} \mathfrak{s c a r} \mathfrak{r}_{\Lambda}^{(E)}$ and its invariant metric summarized in table 6 . Here and in the following, we raise and lower indices of the dual elements using $\delta_{a b}$ and its inverse. Notice that our extension is precisely of the form discussed in Theorem 1 , with $\mathfrak{h}$ being simple and $\mathfrak{g}$ an abelian algebra.

One could have arrived at the double extension of (A)dS-Carroll also by exchanging the rôle of boosts and spatial translations in the Maxwell algebra of table 5 .

The algebra $(\mathfrak{a}) \mathfrak{d} \mathfrak{s c a r}{ }_{\Lambda}^{(E)}$ appears to have gone unnoticed in the literature. We will add some remarks concerning possible interpretations of the algebra in the discussion, section 9 .

\subsection{Metric galilean algebras}

Let us now turn to the (non-torsional) galilean algebras. These do not admit an invariant metric for any value of the cosmological constant $\Lambda$. This is straightforward to check by applying the criterion (3.6) to the commutation relations obtained in the limit $c_{i} \rightarrow 0$ from table 4 , setting contraction parameter $\tau$ to unity and disregarding the trivial central extension $\mathrm{M}$.

In order to initiate the double extension process we have to choose a subalgebra $\mathfrak{g}$. The smallest subalgebra that can be equipped with an $\mathfrak{h}$ invariant metric is the abelian algebra

\footnotetext{
${ }^{16}$ The Carroll algebra can have a nondegenerate metric in three dimensions precisely because it is a coadjoint extension.
} 


\begin{tabular}{|c|c|c|c|}
\hline \multicolumn{2}{|r|}{$(\mathfrak{a}) \mathfrak{d} \mathfrak{s c a r}{ }_{\Lambda}^{(E)}$} & & \\
\hline$\left[\mathrm{J}_{a b}, \mathrm{X}_{c}\right]$ & $-2 \delta_{c[a} \mathrm{X}_{b]}$ & \multicolumn{2}{|r|}{$(\mathfrak{a}) \mathfrak{d} \mathfrak{s c a r}_{\Lambda}^{(E)}$} \\
\hline$\left[\mathrm{J}_{a b}, \mathrm{X}_{c d}\right]$ & $\begin{array}{r}40\left[a \mid\left[c^{\mathbf{X}_{d}} \delta \mid b\right]\right. \\
\delta\end{array}$ & $\left\langle\mathrm{B}_{a}, \mathrm{~B}_{b}\right\rangle$ & $\chi_{\mathrm{B}} \delta_{a b}$ \\
\hline $\begin{array}{l}{\left[\mathrm{B}_{a}, \mathrm{P}_{b}\right]} \\
{[\mathrm{P} \mathrm{H}]}\end{array}$ & $\begin{array}{r}\delta_{a b} \mathrm{H} \\
\Delta^{2} \mathrm{~B}^{2}\end{array}$ & $\langle\mathrm{H}, \mathrm{H}\rangle$ & $\mp \Lambda^{2} \chi_{\mathrm{B}}$ \\
\hline $\begin{array}{l}{[\ulcorner a, \Pi]} \\
{\left[\mathrm{P}_{a}, \mathrm{P}_{b}\right]}\end{array}$ & $\pm \Lambda^{2} \mathrm{~J}_{a b}$ & $\left\langle\mathrm{~J}_{a b}, \mathrm{~J}_{c d}^{*}\right\rangle$ & $2 \delta_{a[c} \delta_{d] b}$ \\
\hline$\left[\mathrm{P}_{a}, \mathrm{P}_{b}^{*}\right]$ & $-\mathrm{J}_{a b}^{*}$ & $\left\langle\mathrm{P}_{a}, \mathrm{P}_{b}^{*}\right\rangle$ & $\delta_{a b}$ \\
\hline$\left[\mathrm{P}_{a}, \mathrm{~J}_{b c}^{*}\right]$ & $\mp 2 \Lambda^{2} \delta_{a[b} \mathrm{P}_{c]}^{*}$ & $\left\langle\mathrm{~J}_{a b}, \mathrm{~J}_{c d}\right\rangle$ & $2 \chi \delta_{a[c} \delta_{d] b}$ \\
\hline$\left[\mathrm{B}_{a}, \mathrm{~B}_{b}\right]$ & $-\chi_{\mathrm{B}} \mathrm{J}_{a b}^{*}$ & $\left\langle\mathrm{P}_{a}, \mathrm{P}_{b}\right\rangle$ & $\mp \chi \Lambda^{2} \delta_{a b}$ \\
\hline$\left[\mathrm{B}_{a}, \mathrm{H}\right]$ & $\pm \Lambda^{2} \chi_{\mathrm{B}} \mathrm{P}_{a}^{*}$ & & \\
\hline
\end{tabular}

Table 6. Double extension based on the AdS-carrollian (upper sign) and dS-carrollian (lower sign) algebra. The vector $\mathrm{X}_{a}$ stands for $\mathrm{B}_{a}, \mathrm{P}_{a}, \mathrm{P}_{a}^{*} ; \mathrm{X}_{a b}$ for $\mathrm{J}_{a b}, \mathrm{~J}_{a b}^{*}$. The metric is nondegenerate for $\chi_{\mathrm{B}} \neq 0$.

spanned by boosts and spatial translations. From invariance with respect to the rotations and nondegeneracy follows that the invariant metric on $\mathfrak{g}=\left\{\mathrm{B}_{a}, \mathrm{P}_{a}\right\}$ is either

$$
\left\langle\mathrm{B}_{a}, \mathrm{~B}_{b}\right\rangle=\chi_{\mathrm{B}} \delta_{a b} \quad\left\langle\mathrm{P}_{a}, \mathrm{P}_{b}\right\rangle=\chi_{\mathrm{P}} \delta_{a b} \quad \chi_{\mathrm{B}} \neq 0 \neq \chi_{\mathrm{P}}
$$

or

$$
\left\langle\mathrm{B}_{a}, \mathrm{P}_{b}\right\rangle=\chi_{\mathrm{BP}} \delta_{a b} \quad \chi_{\mathrm{BP}} \neq 0,
$$

or a combination of both.

Checking for invariance under the action of $\mathrm{H}$, we find that the antidiagonal choice (6.5) is not a viable option. The diagonal option (6.4) on the other hand, obeys condition (4.3) provided that

$$
\chi_{\mathrm{P}} \mp \Lambda^{2} \chi_{\mathrm{B}}=0 .
$$

As in the case of the carrollian algebras this means that this extension exists only for nonvanishing cosmological constant. After double extending we obtain the metric Lie algebra $(\mathfrak{a}) \mathfrak{d} \mathfrak{s g a l}{ }_{\Lambda}^{(E)}$ with commutators and nondegenerate metric summarized in table 7 .

This can be regarded as a generalization of the three-dimensional centrally extended (A)dS-Galilei algebra of table 2 or, more precisely, a generalization of the double extension procedure discussed in section 4.3. Since a non-diagonal metric $\mu_{\mathrm{BP}}$ is not allowed in higher dimensions the linear transformation in equation (4.15) is not necessary.

We want to stress the intriguing observation that the element $\mathrm{H}^{*}$ plays the same rôle as the central extension in the Bargmann algebra, usually called M. We are unaware of previous appearance of this algebra in the literature. We will postpone further comments and interpretations to the discussion section 9 .

\subsection{Metric para-galilean and static algebra}

Even though their Klein pairs and consequently their physics differ, the para-galilean Lie algebra is isomorphic to the Galilei algebra. Thus, the discussion of section 6.4 applies, we 


\begin{tabular}{|c|c|c|c|}
\hline \multicolumn{2}{|r|}{$(\mathfrak{a}) \mathfrak{d} \mathfrak{s g a r} \mathfrak{r}_{\Lambda}^{(E)}$} & \multicolumn{2}{|r|}{$(\mathfrak{a}) \mathfrak{d} \mathfrak{s} \mathfrak{g a r} \mathfrak{l}_{\Lambda}^{(E)}$} \\
\hline$\left[\mathrm{J}_{a b}, \mathrm{X}_{c d}\right]$ & $4 \delta_{[a \mid[c} \mathrm{X}_{d] \mid b]}$ & $\left\langle\mathrm{B}_{a}, \mathrm{~B}_{b}\right\rangle$ & $\chi_{\mathrm{B}} \delta_{a b}$ \\
\hline$\left[\mathrm{J}_{a b}, \mathrm{X}_{c}\right]$ & $-2 \delta_{c[a} \mathrm{X}_{b]}$ & $\left\langle\mathrm{P}_{a}, \mathrm{P}_{b}\right\rangle$ & $\pm \Lambda^{2} \chi_{\mathrm{B}} \delta_{a b}$ \\
\hline$\left[\mathrm{B}_{a}, \mathrm{H}\right]$ & $\mathrm{P}_{a}$ & $\left\langle\mathrm{~J}_{a b}, \mathrm{~J}_{c d}^{*}\right\rangle$ & $2 \delta_{a[c} \delta_{d] b}$ \\
\hline$\left[\mathrm{P}_{a}, \mathrm{H}\right]$ & $\mp \Lambda^{2} \mathrm{~B}_{a}$ & $\left\langle\mathrm{H}, \mathrm{H}^{*}\right\rangle$ & \\
\hline$\left[\mathrm{B}_{a}, \mathrm{~B}_{b}\right]$ & $-\chi_{\mathrm{B}} \mathrm{J}_{a b}^{*}$ & $\langle\mathrm{H}, \mathrm{H}\rangle$ & $\chi_{\mathrm{H}}$ \\
\hline $\begin{array}{l}{\left[\mathrm{P}_{a}, \mathrm{P}_{b}\right]} \\
{\left[\mathrm{B}_{a}, \mathrm{P}_{b}\right]}\end{array}$ & $\begin{array}{r}\mp \Lambda^{2} \chi_{\mathrm{B}} \mathrm{J}_{a b}^{*} \\
\mp \Lambda^{2} \chi_{\mathrm{B}} \delta_{a b} \mathrm{H}^{*}\end{array}$ & $\left\langle\mathrm{~J}_{a b}, \mathrm{~J}_{c d}\right\rangle$ & $2 \chi_{\mathrm{J}} \delta_{a[c} \delta_{d] b}$ \\
\hline
\end{tabular}

Table 7. Double extension based on the AdS-galilean (upper sign) and dS-galilean (lower sign) spacetimes in $D+1$ dimensions and its invariant metric. The vector $\mathrm{X}_{a}$ stands for $\mathrm{B}_{a}, \mathrm{P}_{a}$; the tensor $\mathrm{X}_{a b}$ for $\mathrm{J}_{a b}, \mathrm{~J}_{a b}^{*}$. The bilinear form is nondegenerate if $\chi_{\mathrm{B}} \neq 0$.

\begin{tabular}{|lr|}
\hline & $\mathfrak{s t}^{(E)}$ \\
\hline$\left[\mathrm{J}_{a b}, \mathrm{X}_{c d}\right]$ & $4 \delta_{[a \mid[c} \mathrm{X}_{d] \mid b]}$ \\
{$\left[\mathrm{J}_{a b}, \mathrm{X}_{c}\right]$} & $-2 \delta_{c[a} \mathrm{X}_{b]}$ \\
{$\left[\mathrm{B}_{a}, \mathrm{~B}_{b}\right]$} & $-\chi_{\mathrm{B}} \mathrm{J}_{a b}^{*}$ \\
{$\left[\mathrm{P}_{a}, \mathrm{P}_{b}\right]$} & $-\chi_{\mathrm{P}} \mathrm{J}_{a b}^{*}$ \\
{$\left[\mathrm{~B}_{a}, \mathrm{P}_{b}\right]$} & $-\chi_{\mathrm{BP}} \mathrm{J}_{a b}^{*}$ \\
\hline
\end{tabular}

\begin{tabular}{|lr|}
\hline & $\mathfrak{s t}^{(E)}$ \\
\hline$\left\langle\mathrm{B}_{a}, \mathrm{~B}_{b}\right\rangle$ & $\chi_{\mathrm{B}} \delta_{a b}$ \\
$\left\langle\mathrm{P}_{a}, \mathrm{P}_{b}\right\rangle$ & $\chi_{\mathrm{P}} \delta_{a b}$ \\
$\left\langle\mathrm{~B}_{a}, \mathrm{P}_{b}\right\rangle$ & $\chi_{\mathrm{BP}} \delta_{a b}$ \\
$\langle\mathrm{H}, \mathrm{H}\rangle$ & $\chi_{\mathrm{H}}$ \\
$\left\langle\mathrm{J}_{a b}, \mathrm{~J}_{c d}^{*}\right\rangle$ & $2 \delta_{a[c} \delta_{d] b}$ \\
$\left\langle\mathrm{~J}_{a b}, \mathrm{~J}_{c d}\right\rangle$ & $2 \chi_{\mathrm{J}} \delta_{a[c} \delta_{d] b}$ \\
\hline
\end{tabular}

Table 8. Double extension based on the static spacetimes in $D+1$ dimensions and its invariant metric. The vector $\mathrm{X}_{a}$ stands for $\mathrm{B}_{a}, \mathrm{P}_{a}$; the tensor $\mathrm{X}_{a b}$ for $\mathrm{J}_{a b}, \mathrm{~J}_{a b}^{*}$. The bilinear form is nondegenerate if $\chi_{\mathrm{H}}$ and $\left(\chi_{\mathrm{B}} \chi_{\mathrm{P}}-\chi_{\mathrm{BP}}^{2}\right)$ are nonzero.

merely need to exchange the boosts and spatial translations. This means the metric paragalilean algebra is given by the coadjoint extension, for which we again refer to section 7 .

For completeness we also provide the double extension of the static algebra in table 8 , where $\mathfrak{g}=\left\{\mathrm{B}_{a}, \mathrm{P}_{a}, \mathrm{H}\right\}$ and $\mathfrak{h}=\left\{\mathrm{J}_{a b}\right\}$. This is the choice with the smallest number of additional generators.

\subsection{Non-contracting algebras}

For completeness, let us also study higher-dimensional generalizations of the noncontracting algebras of section 5 regarding the existence of invariant metrics. For those algebras without invariant metric we construct again the double extension with the smallest number of additional generators.

\subsubsection{Torsional galilean algebras}

We saw in section 5.1 that the three-dimensional torsional galilean theories do not support a nondegenerate metric, and the only possible double extension is the coadjoint extension. We will find that this pattern generalizes to higher dimensions. 
The commutation relations of the torsional galilean algebras are given by

$$
\begin{aligned}
& {\left[\mathrm{J}_{a b}, \mathrm{~J}_{c d}\right]=4 \delta_{[a \mid[c} \mathrm{J}_{b] \mid d]} \quad\left[\mathrm{J}_{a b}, \mathrm{P}_{c}\right]=-2 \delta_{c[a} \mathrm{P}_{b]} \quad\left[\mathrm{J}_{a b}, \mathrm{~B}_{c}\right]=-2 \delta_{c[a} \mathrm{B}_{b]},} \\
& {\left[\mathrm{H}, \mathrm{B}_{a}\right]=-\mathrm{P}_{a} \quad\left[\mathrm{H}, \mathrm{P}_{a}\right]=\alpha \mathrm{B}_{a}+\beta \mathrm{P}_{a}}
\end{aligned}
$$

with $\alpha=\gamma, \beta=(1+\gamma), \gamma \in[-1,1)$ for torsional galilean-dS and $\alpha=\left(1+\chi^{2}\right), \beta=2 \chi, \chi>0$ for torsional galilean-AdS. These algebras do not have an invariant metric.

The only subalgebras compatible with the double extension structure are either $\mathfrak{g}=$ $\left\{\mathrm{H}, \mathrm{P}_{a}, \mathrm{~B}_{a}\right\}$ or $\mathfrak{g}=\left\{\mathrm{P}_{a}, \mathrm{~B}_{a}\right\}$. However, in both cases the requirement of invariance under $\mathrm{H}$ and $\mathrm{J}_{a}$ leads to a degenerate metric $\Omega^{\mathfrak{g}}$. As in $2+1$ dimensions, the only possibility is therefore the coadjoint extension, which we refrain from writing, with its associated nondegenerate metric.

\subsubsection{Carrollian light cone algebra}

The carrollian light cone algebra in $D+1$ is given by

$$
\begin{aligned}
& {\left[\mathrm{J}_{a b}, \mathrm{~J}_{c d}\right]=4 \delta_{[a \mid[c} \mathrm{J}_{b] \mid d]} \quad\left[\mathrm{J}_{a b}, \mathrm{P}_{c}\right]=-2 \delta_{c[a} \mathrm{P}_{b]} \quad\left[\mathrm{J}_{a b}, \mathrm{~B}_{c}\right]=-2 \delta_{c[a} \mathrm{B}_{b]},} \\
& {\left[\mathrm{H}, \mathrm{B}_{a}\right]=\mathrm{B}_{a} \quad\left[\mathrm{H}, \mathrm{P}_{a}\right]=-\mathrm{P}_{a} \quad\left[\mathrm{~B}_{a}, \mathrm{P}_{b}\right]=\mathrm{H} \delta_{a b}+\mathrm{J}_{a b} .}
\end{aligned}
$$

This is isomorphic to $\mathfrak{s o}(D+1,1)$. A one-parameter family of nondegenerate metrics exists due to existence of a nondegenerate Killing form.

\subsubsection{Aristotelian algebras}

As in the three-dimensional case, for these algebras the boosts do not act effectively on the underlying homogeneous space. We have therefore quotiented by the boosts such that the kinematical algebra is spanned only by $\left\{\mathrm{J}_{a b}, \mathrm{P}_{a}, \mathrm{H}\right\}$, and the kinematical brackets common to all aristotelian algebras are given by

$$
\left[\mathrm{J}_{a b}, \mathrm{~J}_{c d}\right]=4 \delta_{[a \mid[c} \mathrm{J}_{b] \mid d]} \quad\left[\mathrm{J}_{a b}, \mathrm{P}_{c}\right]=-2 \delta_{c[a} \mathrm{P}_{b]} .
$$

Let us summarize the results for these algebras:

- The static aristotelian algebra that is completely defined by the commutation relations (6.9) has no nondegenerate invariant metric. However, one can perform a double extension choosing the abelian algebra $\mathfrak{g}=\left\{\mathrm{H}, \mathrm{P}_{a}\right\}$. Introducing the elements $\mathrm{J}_{a b}^{*}$ dual to the rotations $\mathrm{J}_{a b}$, we obtain a Lie algebra, we denote by $\mathfrak{a r s t}^{(E)}$, with the additional brackets

$$
\left[\mathrm{J}_{a b}, \mathrm{~J}_{c d}^{*}\right]=4 \delta_{[a \mid[c} \mathrm{J}_{b] \mid d]}^{*} \quad\left[\mathrm{P}_{a}, \mathrm{P}_{b}\right]=-\mu_{\mathrm{P}} \mathrm{J}_{a b}^{*} .
$$

and invariant metric

$$
\langle\mathrm{H}, \mathrm{H}\rangle=\mu_{\mathrm{H}} \quad\left\langle\mathrm{P}_{a}, \mathrm{P}_{b}\right\rangle=\mu_{\mathrm{P}} \delta_{a b} \quad\left\langle\mathrm{~J}_{a b}, \mathrm{~J}_{c d}^{*}\right\rangle=2 \delta_{a[b} \delta_{c] d} .
$$

This is the generalization of the three-dimensional case in section 5.3.1 where $\mathrm{J}^{*}$ becomes central. 
- The torsional static aristotelian algebra, having the additional bracket

$$
\left[\mathrm{H}, \mathrm{P}_{a}\right]=\mathrm{P}_{a}
$$

has no invariant metric. In contrast to the static case, but again similar to all other cases with torsion, the only possible double extension is based on the coadjoint algebra since the bracket (6.12) is incompatible with having a nondegenerate bilinear form. We find the additional brackets

$$
\left[\mathrm{J}_{a b}, \mathrm{~J}_{c d}^{*}\right]=4 \delta_{[a \mid[c} \mathrm{J}_{b] \mid d]}^{*} \quad\left[\mathrm{P}_{a}, \mathrm{P}_{b}^{*}\right]=-\mathrm{J}_{a b}^{*} \quad\left[\mathrm{H}, \mathrm{P}_{a}^{*}\right]=-\mathrm{P}_{a}^{*} \quad\left[\mathrm{~J}_{a b}, \mathrm{P}_{c}^{*}\right]=-2 \delta_{c[a} \mathrm{P}_{b]}^{*}
$$

and the invariant metric induced from the coadjoint extension structure.

- The aristotelian algebras $\mathbb{R} \times \mathbb{H}^{D}$ and $\mathbb{R} \times \mathbb{S}^{D}$ are defined by

$$
\left[\mathrm{P}_{a}, \mathrm{P}_{b}\right]= \pm \mathrm{J}_{a b}
$$

with the upper sign for the former algebra. These algebras are isomorphic to a direct sum of an abelian factor generated by $\mathrm{H}$ and the simple factor $\mathfrak{s o}(D, 1)$ and $\mathfrak{s o}(D+$ $1)$, respectively. The kinematical algebra has therefore a two-parameter family of invariant metrics, the metric of the simple part being proportional to the Killing form.

\section{The coadjoint kinematical algebras and their limits}

In the previous section we examined all higher-dimensional kinematical spacetimes regarding the existence of an invariant metric for their kinematical algebra. For those algebras without invariant metric we found appropriate double extensions that have an invariant metric by construction. However, the resulting metric Lie algebras do not all have the same dimension, in particular those of the cube of Bacry-Lévy-Leblond. If we are interested in considering limits for these higher-dimensional kinematic algebras with invariant metric and putative theories based on them, we have to consider the coadjoint extensions for all algebras. This way we obtain limits for which the invariant metric stays nondegenerate and which generalizes the outer cube of figure 1 to higher-dimensional kinematical algebras.

As we mentioned in section 4.1 the coadjoint Lie algebra is $\mathfrak{c o a d}=D(0, \mathfrak{h})$ defined on the vector space $\mathfrak{h} \dot{+} \mathfrak{h}^{*}$ (spanned by $\mathrm{H}_{\alpha}$ and $\mathrm{H}^{* \alpha}$, respectively) by

$$
\begin{aligned}
{\left[\mathrm{H}_{\alpha}, \mathrm{H}_{\beta}\right] } & =f_{\alpha \beta}{ }^{\gamma} \mathrm{H}_{\gamma} \\
{\left[\mathrm{H}_{\alpha}, \mathrm{H}^{* \beta}\right] } & =-f_{\alpha \gamma}{ }^{\beta} \mathrm{H}^{* \gamma} \\
{\left[\mathrm{H}^{* \alpha}, \mathrm{H}^{* \beta}\right] } & =0
\end{aligned}
$$

and the invariant metric

$$
\Omega_{a b}^{\mathfrak{c o a d}}=\begin{aligned}
& \mathrm{H}_{\alpha} \\
& \mathrm{H}^{* \alpha}
\end{aligned}\left(\begin{array}{cc}
h_{\alpha \beta} & \delta_{\alpha}^{* \beta} \\
\delta^{\alpha}{ }_{\beta} & 0
\end{array}\right) .
$$




\begin{tabular}{|lrlr|}
\hline \multicolumn{4}{|c|}{$D\left(0,(\mathfrak{a}) \mathfrak{d} \mathfrak{s}_{\tau, c_{i}, \Lambda}\right)$} \\
\hline$\left[\mathrm{J}_{a b}, \mathrm{X}_{c}^{*}\right]$ & $-2 \delta_{c[a} \mathrm{X}_{b]}^{*}$ & {$\left[\mathrm{H}, \mathrm{P}_{a}^{*}\right]$} & $\tau^{2} \mathrm{~B}_{a}^{*}$ \\
{$\left[\mathrm{~J}_{a b}, \mathrm{~J}_{c d}^{*}\right]$} & $4 \delta_{[a \mid[c} \mathrm{J}_{d] \mid b]}^{*}$ & {$\left[\mathrm{H}, \mathrm{B}_{a}^{*}\right]$} & $\mp \Lambda^{2} \mathrm{P}_{a}^{*}$ \\
{$\left[\mathrm{~B}_{a}, \mathrm{~J}_{b c}^{*}\right]$} & $-2 \tau^{2} c_{i}^{2} \delta_{a[b} \mathrm{B}_{c]}^{*}$ & {$\left[\mathrm{P}_{a}, \mathrm{~J}_{b c}^{*}\right]$} & $\mp 2 \Lambda^{2} c_{i}^{2} \delta_{a[b} \mathrm{P}_{c]}^{*}$ \\
{$\left[\mathrm{~B}_{a}, \mathrm{P}_{b}^{*}\right]$} & $-\tau^{2} \delta_{a b} \mathrm{H}^{*}$ & {$\left[\mathrm{P}_{a}, \mathrm{~B}_{b}^{*}\right]$} & $\pm \Lambda^{2} \delta_{a b} \mathrm{H}^{*}$ \\
{$\left[\mathrm{~B}_{a}, \mathrm{~B}_{b}^{*}\right]$} & $-\mathrm{J}_{a b}^{*}$ & {$\left[\mathrm{P}_{a}, \mathrm{P}_{b}^{*}\right]$} & $-\mathrm{J}_{a b}^{*}$ \\
{$\left[\mathrm{~B}_{a}, \mathrm{H}^{*}\right]$} & $-c_{i}^{2} \mathrm{P}_{a}^{*}$ & {$\left[\mathrm{P}_{a}, \mathrm{H}^{*}\right]$} & $c_{i}^{2} \mathrm{~B}_{a}^{*}$ \\
{$\left[\mathrm{~B}_{a}, \mathrm{M}^{*}\right]$} & $-\alpha \mathrm{P}_{a}^{*}$ & {$\left[\mathrm{P}_{a}, \mathrm{M}^{*}\right]$} & $\alpha \mathrm{B}_{a}^{*}$ \\
\hline
\end{tabular}

\begin{tabular}{|lr|}
\hline & $D\left(0,(\mathfrak{a}) \mathfrak{d} \mathfrak{s}_{\tau, c_{i}, \Lambda}\right)$ \\
\hline$\left\langle\mathrm{J}_{a b}, \mathrm{~J}_{c d}^{*}\right\rangle$ & $2 \mu^{*} \delta_{a[c} \delta_{d] b}$ \\
$\left\langle\mathrm{~B}_{a}, \mathrm{~B}_{b}^{*}\right\rangle$ & $\mu^{*} \delta_{a b}$ \\
$\left\langle\mathrm{H}, \mathrm{H}^{*}\right\rangle$ & $\mu^{*}$ \\
$\left\langle\mathrm{P}_{a}, \mathrm{P}_{b}^{*}\right\rangle$ & $\mu^{*} \delta_{a b}$ \\
$\left\langle\mathrm{M}, \mathrm{M}^{*}\right\rangle$ & $\mu^{*}$ \\
\hline
\end{tabular}

Table 9. Additional commutation relations to table 4 for the coadjoint extension of the AdS (upper sign) and dS (lower sign) algebra in $D+1$ dimensions. The vector $\mathrm{X}_{a}^{*}$ stands for $\mathrm{B}_{a}^{*}$ and $\mathrm{P}_{a}^{*}$; $\mathrm{X}_{a}$ for $\mathrm{B}_{a}$ and $\mathrm{P}_{a}$. The algebra and its metric stay regular for all possible contractions.

We take $\mathfrak{h}$ to be the centrally extended (A)dS algebra of table 4 with its associated metric $h_{\alpha \beta}$, that becomes degenerate in the various limits. Introducing the dual generators of $\mathfrak{h}^{*}$ and using the coadjoint construction one arrives at the coadjoint algebra summarized in table 9. We display only the additional relations coming from the double extension, all other commutators and matrix elements are identical to those in table 4.

Even though the metric $h_{\alpha \beta}$ becomes degenerate in the IW contractions the full invariant metric (7.2) stays nondegenerate. The reason for this is that the rescalings of equation (A.5) that were used to arrive at table 4 are counterbalanced by the inverse rescalings for the dual elements. This guarantees both nondegeneracy of the metric and regularity of the contraction [71]. This inverse rescaling of the dual elements is already accounted for when starting directly from the commutation relations in table 4 .

The invariant metric is nondegenerate for $\mu^{*} \neq 0$. Due to our construction all limits are well-defined. Notice that the element $\mathrm{M}^{*}$ dual to $\mathrm{M}$ is trivial as it can be absorbed in a shift of $\mathrm{H}^{*}$. However, in the nonrelativistic limit it is nontrivial.

These contractions can be seen as the generalization of the cube of Bacry-LévyLeblond [38] to metric Lie algebras and spacetimes. Indeed, the contractions commute and the invariant metric stays nondegenerate.

\section{Summary of results}

This work concerns the classification of gravitational theories in $2+1$ dimensions and limits of their actions. We analyzed the underlying structure and the possibility for higherdimensional generalizations.

The starting point was the restriction to spatially isotropic spacetimes, i.e., spacetimes with rotational, spatial and temporal translations, with boosts (kinematical) and without boosts (aristotelian) [38, 40]. This is the natural generalization of the maximally symmetric (A)dS and Minkowski spacetimes, when the necessity of an invariant tangent space metric is dropped. The spacetime provides us with a Lie algebra $\mathfrak{k}$ and a subalgebra $\mathfrak{b}$, the Klein pair $(\mathfrak{k}, \mathfrak{b})$, which fixes the physical interpretation of the generators of the algebra. This is 


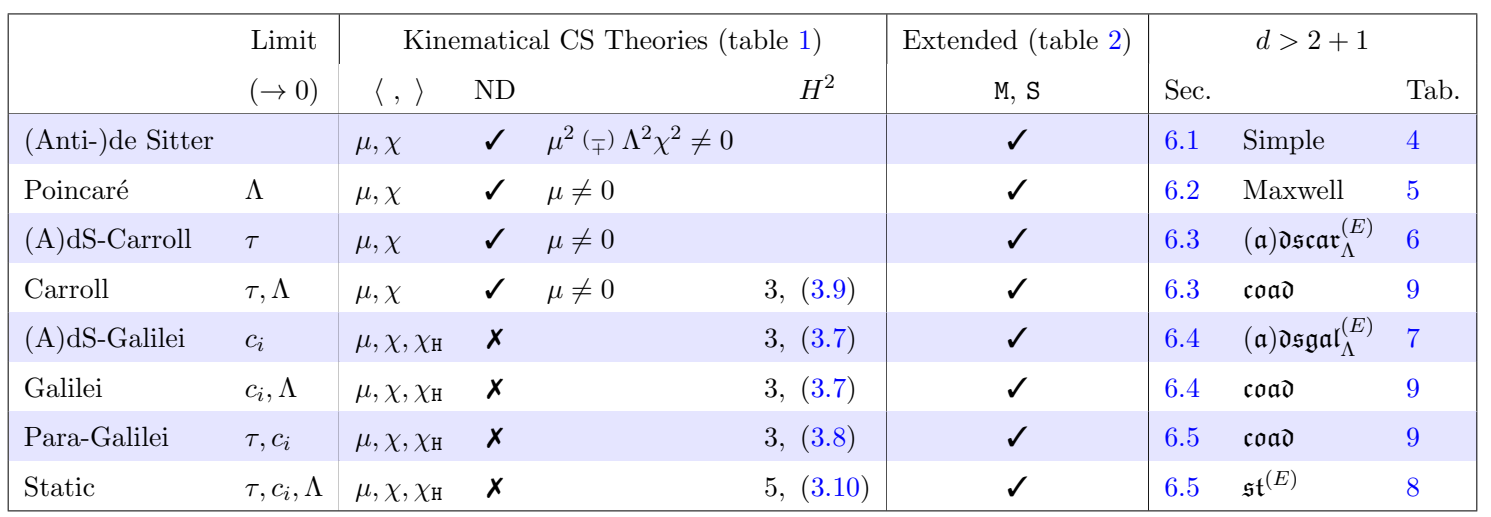

Table 10. Chern-Simons theories that arise from a limit of (A)dS.

the starting point for the gauging of our theories. The gauging provides us with equations of motion for the vielbein and connection of our gravitational theory. It does however not provide us necessarily with a well-defined Chern-Simons action principle, which, among other useful properties, is the most bullet-proof starting point for the quantization of a theory.

This led us to analyze the existence of invariant metrics and central extensions of the underlying Lie algebras, both of which we provide in full generality (which closes the remaining gaps in the literature). Since, e.g., the nonrelativistic theories do not provide us with such a well-defined theory, and motivated by earlier work [31, 33-35, 37, 48], we also fully analyzed the existence of invariant metrics when the central extensions are added.

For the theories which follow as a limit of $(\mathrm{A}) \mathrm{dS}_{3}$ gravity we then show that, when two central extensions are carefully added in the right way, all limits are well defined also for the action and organize themselves as a tesseract presented in figure 1. The (A)dS-carrollian theories and their limit seem to have been unnoticed so far.

For the remaining non-contracting cases we introduced the structure that organizes the non-reductive Lie algebras with invariant metrics, double extensions. This demystifies the existence of the nondegenerate "Killing form" for non-semisimple algebras and made it possible for us to analyze the detailed structure and paved the way to our main result: We provide for every kinematical and aristotelian spacetime a well-defined Chern-Simons theory (with the minimal number of additional fields).

We then analyzed if these metric Lie algebras generalize to higher dimensions (section 6), recovering the well-known Maxwell algebra [67,68] as a double extension, and various novel ones, most notably the metric (A)dS-galilean and (A)dS-carrollian algebras. Finally, we provide a generalization of the cube to any dimension (section 7).

Before we move on to a more detailed discussion of our results let us emphasize that our results can be easily adapted to also include the riemannian "spacetimes", see appendix A.3.

Let us now provide an overview of the theories that admit a limit from (A)d $\mathrm{S}_{3}$ gravity. The vertical rules of table 10 separate the Chern-Simons theories without central extensions (table 1) from the ones with two, in some cases trivial, central extension (table 2). The last column concerns the higher-dimensional generalization. 


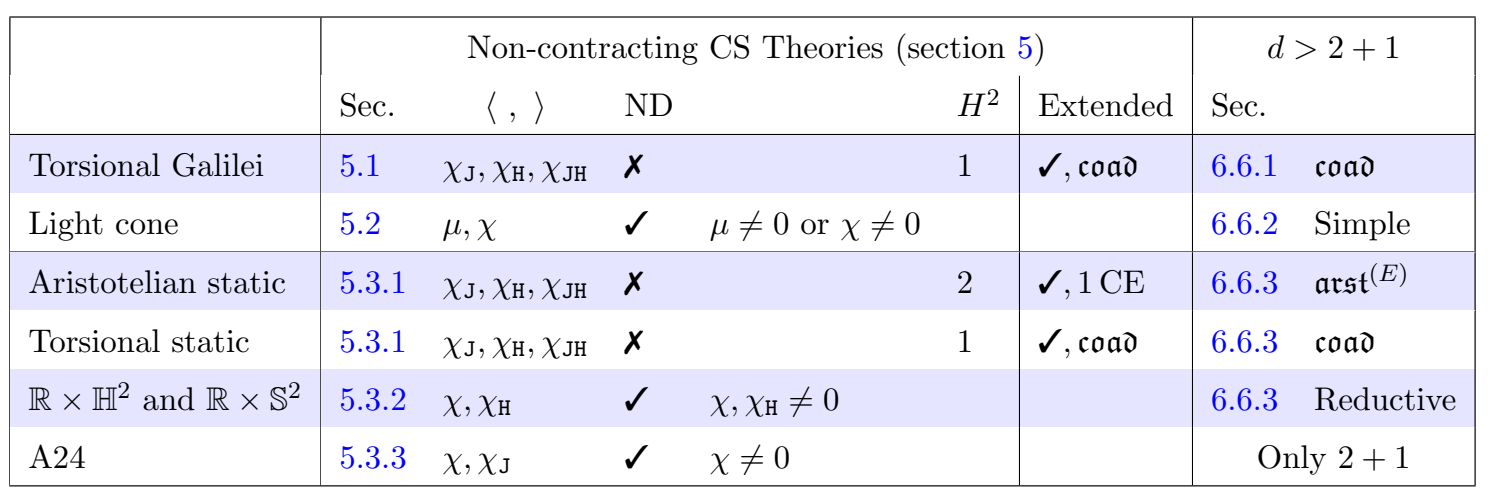

Table 11. Non-contracting Chern-Simons theories.

For the nonextended theories the limits of table 1 provide the most general theory except for the freedom to add a $\langle\mathrm{H}, \mathrm{H}\rangle=\chi_{\mathrm{H}}$ term for the nonrelativistic theories. In the ND column we have marked the theories that admit a nondegenerate $(\boldsymbol{V})$ or degenerate $(\boldsymbol{x})$ invariant metric and the condition for nondegeneracy. This aligns with the inner cube of figure 1. $H^{2}$ denotes how many nontrivial central extensions are possible and a link to their commutators. In section 3.2 the possible invariant metrics for the centrally extended cases are discussed in detail.

Adding two central extensions ( $\mathrm{M}$ and $\mathrm{S}$ ) leads to the limits of table 2, for which all theories admit a nondegenerate invariant metric as long as (3.11) is fulfilled. This is also represented in the outer cube of figure 1 .

In section 6 we analyzed if the underlying metric Lie algebras generalize to higher dimensions. We again describe how each Lie algebra needs to be altered (if at all) so that we are able to equip it with an invariant metric. Some correspond precisely to the higherdimensional analog of the lower dimensional case ((A)dS, and (A)dS-Galilei, static) some are a generalization (Maxwell, (A)dS Carroll), while for the remaining cases we need to fall back to the trivial solution of providing them with the coadjoint Lie algebra.

Table 11 provides the information concerning the Chern-Simons theories that do not arise from a limit (middle column) and their higher-dimensional generalizations (right column).

The Chern-Simons theories for the light cone, $\mathbb{R} \times \mathbb{H}^{2}, \mathbb{R} \times \mathbb{S}^{2}$ and A24 are well-defined from the start $(\mathcal{V})$, whereas for the aristotelian static case the addition of one central extension suffices. For the remaining ones we have to resort to the coadjoint Lie algebras $(\mathfrak{c o a d j})$. With $H^{2}$ we again indicate the existence of nontrivial central extension of which the precise form can be found in the respective sections.

For the higher dimensional metric Lie algebras the only nontrivial case is the aristotelian static Lie algebra which is a true generalization of the lower dimensional one.

More details can be found following the links to the respective sections (denoted by sec.). 


\section{Discussion}

We want to finish by drawing attention to some intriguing aspects of our classification, mostly focusing on the non-lorentzian theories or generic features.

\subsection{Three-dimensional kinematical theories}

From the $(2+1)$-dimensional point of view it is interesting to investigate the limit with the AdS/CFT correspondence in mind, i.e., the limit from $\mathrm{AdS}_{3}$ to AdS-Carroll or AdSGalilei. One might hope that the presence of the cosmological constant again translates into beneficial infrared regulating properties, similarly to AdS. In this sense these theories also present themselves as interesting testing ground for the calculations of partition functions [72, 73] (see also [74-76], or [77] for the flat case) and for sharpening our understanding of entanglement entropy, following, e.g., [78, 79], in more general set-ups.

Important for this endeavor would be a deeper understanding of the solution space, boundary conditions and their asymptotic symmetries, i.e., hints of possible dual theories. While there has been a lot of progress for the lorentzian theories, less has been done for their non-lorentzian cousins, see however [31, 80] for the carrollian and $[31,33-35,48]$ for the galilean case (see also for related boundary conditions beyond the kinematical set-up [48]).

Concerning possible boundary conditions let us add that following $[2,8]$ all models with an invariant metric can be equipped with boundary conditions leading to WZW theories on the boundary (for recent work see also [81]). Remarkably, similar to the well-known WZW models based on simple Lie algebras, also the ones based on the metric Lie algebras permit a Sugawara construction [82] and are therefore conformal field theories. For the calculation of the central charge in terms of the level of the WZW model the double extension structure plays a fundamental rôle [83] and should be tractable.

It is interesting that the carrollian Chern-Simons theories are, like their lorentzian cousins, well-defined from the start. Certainly, the connection of the ultrarelativistic limit to the strong coupling limit of general relativity and the Hamiltonian formulation [84] deserves further attention.

Since the homogeneous carrollian spacetimes are null surfaces of Minkowski or (A)dS spaces [40] (see also [52]), it is tempting to speculate that these carrollian $2+1$ ChernSimons theories (in particular the theory based on the carrollian light cone) are connected to edge states and BMS symmetries [27, 28] in $3+1$ dimensions. An intriguing hint is that the invariant tensors of the tangent space of these theories, given by the carrollian degenerate metric and a carrollian vector field, provide the right carrollian structure [29]. These theories might also yield an interesting set-up, with manifest carrollian symmetries in the bulk, for fluid/gravity holographic models [85].

An obvious generalization is to consider supersymmetric CS theories. The tools employed here and in [31], should translate to the supersymmetric case and could clarify the rôle of the nonrelativistic supergravity theory of $[35] .^{17}$

\footnotetext{
${ }^{17}$ While the Medina-Revoy theorem has not been proven for Lie superalgebras, double extensions still work [43].
} 
Furthermore, the double extension structure underlying metric Lie algebras can be helpful in understanding Chern-Simons theories based on algebras that do not belong to the class of kinematical Lie algebras discussed in this work, e.g., $[49,86]$.

Similar to the way we classified all three-dimensional Chern-Simons theories in this work it is possible to carry this framework to two dimensional dilaton gravity theories. For linear dilaton potentials these gravity theories can be written as BF theories. Given the classification of two-dimensional kinematical spacetimes in [40] it should be straightforward to similarly classify all two-dimensional BF theories for these spacetimes. Since the BF formulation of the Jackiw-Teitelboim model makes apparent the connection to the Schwarzian action $[87,88]$, that rose to prominence due to its relation to the SYK model [89], these models could have potentially interesting applications regarding generalizations of the SYK model.

\subsection{Higher dimensions}

Due to their connection to Newton-Cartan/Hořava-Lifshitz gravity [35, 36] there has been interest in higher-dimensional generalizations of the double extended galilean Chern-Simons theory [90, 91]. It was argued, from rather different angles, that the actions of [90, 91], provide exactly that.

Here we provide another complementary point of view: while the metric Lie algebra for the flat galilean Lie algebra does not generalize, it is intriguing that it does for nonvanishing cosmological constant to the novel $(\mathfrak{a}) \mathfrak{d} \mathfrak{s g a l}{ }_{\Lambda}^{(E)}$ algebras (table 7$)$. Let us also emphasize that the double extension enforces the existence of the central Bargmann element exactly at the right place for it to be the "mass". We find these observations to be an intriguing and surprising result.

Another place where one might hope for this symmetries to emerge, due to the fact that they arise in a similar way to the Maxwell algebra, is for galilean or carrollian electrodynamics in a homogeneous field [92].

Let us finally note that for the flat galilean metric Lie algebra, like [90], we also need to double the dimension of the algebra. However, as can be easily checked by the criteria of section 3.2, the algebra in [90] is not metric, which does not prevent the authors from writing an action principle. For a natural generalization of a Chern-Simons theory, i.e., a theory that is invariant under the full galilean symmetries, our model might be an interesting candidate, e.g., in the BF theory formulation.

\section{Acknowledgments}

SP is grateful to José Figueroa-O'Farrill for insightful discussions and collaboration on kinematical structures. We thank Glenn Barnich, Oscar Fuentealba, Joaquim Gomis, Hernán González, Daniel Grumiller, Marc Henneaux, Victor Lekeu, Wout Merbis, Niels Obers, Gerben Oling, Jan Rosseel, and Friedrich Schöller for insightful discussions. We also acknowledge useful feedback from the anonymous referees.

The research of JM and SP is supported by the ERC Advanced Grant "High-SpinGrav" and by FNRS-Belgium (Convention FRFC PDR T.1025.14 and Convention IISN 4.4503.15). JS is supported by the ERC Advanced Grant GravBHs-692951. 
SP would like to express a special thanks to the Mainz Institute for Theoretical Physics (MITP) for its hospitality and support during the MITP Topical Workshop "Applied Newton-Cartan Geometry" (APPNC2018), where a preliminary version of our results was presented. The authors acknowledge support from the Erwin Schrödinger Institute during their stay at the "Higher Spins and Holography" workshop.

SP dedicates this work to Christa Zauner who is now "auf der anderen Seite des Weges".

\section{A Conventions}

For our kinematical Lie algebras and spacetimes we use the following conventions. Upper case Latin indices denote spacetime indices, while lower case ones denote spatial indices:

$$
A=(\overbrace{0, \underbrace{1, \ldots, D}_{a, b, \ldots}}^{\mu, \nu, \ldots}, \overbrace{D+1}^{\natural}) \quad \eta_{A B}=\operatorname{diag}(\overbrace{\eta_{00}, \delta_{a b}}^{\eta_{\mu \nu}}, \eta_{\text {吼 }}) .
$$

and $\epsilon_{012 \cdots D+1}=1$.

Depending on the sign of $\eta_{00}$ we are considering either lorentzian $\left(\eta_{00}=-1\right)$ or Euclidean geometries $\left(\eta_{00}=+1\right)$. Throughout the main text we discuss only the former, but see appendix A.3. The sign of $\eta_{\text {如 }}$ determines the sign of the cosmological constant with anti-de Sitter for $\eta_{\text {仲 }}=-1$ and de Sitter for $\eta_{\text {仲 }}=+1$. We will discuss these two cases in parallel, which leads to \pm and $\mp$ signs, of which the upper sign always refers to anti-de Sitter and the lower to de Sitter. The Lie algebras are, if not said otherwise, real and their vector space elements are denoted in typewriter font, like, e.g., X, Y.

The algebra that leaves $\eta_{A B}$ invariant is then given by

$$
\begin{aligned}
{\left[\mathrm{M}_{A B}, \mathrm{M}_{C D}\right] } & =4 \eta_{[A \mid[C} \mathrm{M}_{D] \mid B]}=\eta_{A C} \mathrm{M}_{D B}-\eta_{A D} \mathrm{M}_{C B}-\eta_{B C} \mathrm{M}_{D A}+\eta_{B D} \mathrm{M}_{C A} \\
\left\langle\mathrm{M}_{A B}, \mathrm{M}_{C D}\right\rangle & =2 \mu \eta_{A[C} \eta_{D] B}=\mu\left(\eta_{A C} \eta_{D B}-\eta_{A D} \eta_{C B}\right)
\end{aligned}
$$

and can also be written, using $\hat{\mathrm{J}}_{\mu \nu} \equiv \mathrm{M}_{\mu \nu}$ and $\hat{\mathrm{P}}_{\mu} \equiv \mathrm{M}_{\mu \natural}$, as

$$
\begin{aligned}
{\left[\hat{\mathrm{J}}_{\mu \nu}, \hat{\mathrm{J}}_{\rho \lambda}\right] } & =4 \eta_{\left[\mu \mid\left[\rho \hat{\mathrm{J}}_{\lambda] \mid \nu]}\right.\right.} \\
{\left[\hat{\mathrm{J}}_{\mu \nu}, \hat{\mathrm{P}}_{\rho}\right] } & =-2 \eta_{\rho[\mu} \hat{\mathrm{P}}_{\nu]} \\
{\left[\hat{\mathrm{P}}_{\mu}, \hat{\mathrm{P}}_{\nu}\right] } & = \pm \hat{\mathrm{J}}_{\mu \nu}
\end{aligned}
$$

with invariant metric

$$
\begin{aligned}
\left\langle\hat{\mathrm{J}}_{\mu \nu}, \hat{\mathrm{J}}_{\rho \lambda}\right\rangle & =2 \mu \eta_{\mu[\rho} \eta_{\lambda] \nu} \\
\left\langle\hat{\mathrm{J}}_{\mu \nu}, \hat{\mathrm{P}}_{\rho}\right\rangle & =0 \\
\left\langle\hat{\mathrm{P}}_{\mu}, \hat{\mathrm{P}}_{\nu}\right\rangle & =\mp \mu \eta_{\mu \nu} .
\end{aligned}
$$

In order to take the contractions leading to the kinematical algebras of [38], we find it useful to decompose the generators further, and introduce the contraction parameters $c_{i}, \tau$, and $\Lambda$. We define $\mathrm{J}_{a b} \equiv \hat{\mathrm{J}}_{a b}, \mathrm{~B}_{a} \equiv \hat{\mathrm{J}}_{0 a}, \mathrm{P}_{a} \equiv \hat{\mathrm{P}}_{a}, \mathrm{H} \equiv \hat{\mathrm{P}}_{0}$ and introduce an additional 
(central) element M. The generators appropriate for the contracted algebras are obtained after the following isomorphism:

$$
\begin{aligned}
g_{\Lambda, \tau, c_{i}} \mathrm{~B}_{a} & =\left(\tau c_{i}\right)^{-1} \mathrm{~B}_{a} & g_{\Lambda, \tau, c_{i}} \mathrm{P}_{a} & =\left(\Lambda c_{i}\right)^{-1} \mathrm{P}_{a} \\
g_{\Lambda, \tau, c_{i}} \mathrm{H} & =(\Lambda \tau)^{-1}\left(\mathrm{H}+\alpha c_{i}^{-2} \mathrm{M}\right) & g_{\Lambda, \tau, c_{i}} \mathrm{M} & =\left(\Lambda \tau c_{i}^{2}\right)^{-1} \mathrm{M},
\end{aligned}
$$

which leads to commutation relations and invariant metric summarized in table 4.

\section{A.1 Conventions for $2+1$ dimensions}

For $2+1$ dimensions $(D=2)$ and restricting to $\eta_{00}=-1$ we have

$$
\eta_{\mu \nu}=\operatorname{diag}(-,+,+) \quad \eta_{a b}=\delta_{a b}=\operatorname{diag}(+,+) .
$$

For the Levi-Civita symbol we define

$$
\epsilon_{a b} \equiv \epsilon_{0 a b} \quad \epsilon_{012}=\epsilon_{12}=1 \quad \epsilon^{a b}=\epsilon_{a b} .
$$

We dualize using

$$
\hat{\mathrm{J}}_{\mu}=-\frac{1}{2} \epsilon_{\mu}{ }^{\nu \rho} \hat{\mathrm{J}}_{\nu \rho} \quad \Longleftrightarrow \quad \hat{\mathrm{J}}_{\mu \nu}=\epsilon_{\mu \nu}{ }^{\rho} \hat{\mathrm{J}}_{\rho}
$$

to arrive at (2.3) $\left(\epsilon_{\mu \nu}{ }^{\rho}=\epsilon_{\mu \nu \lambda} \eta^{\lambda \rho}\right)$. This implies that $\mathrm{J}_{a b}=-\epsilon_{a b} \mathrm{~J}^{\mathrm{d}}$ and $\mathrm{B}_{a}=\epsilon_{a b} \mathrm{~B}_{b}^{\mathrm{d}}$, where on the left-hand side one has the non-dualized generators. In sections 2,4 , and 5 we use the dualized operators only and consistently drop the superscript d.

\section{A.2 Most general centrally extended (A)dS}

In order to arrive at table 2 we write the commutation relations (2.3) using the space-time split (3.1) (this is equivalent to table 1 setting all contraction parameters to unity) and add two $\mathfrak{u}(1)$ generators $\mathrm{M}$ and $\mathrm{S}$ commuting with all other elements of the algebra. The invariant bilinear form for the (A)dS part is taken to be given by (2.5), and we introduce the additional parameters $\mu_{\mathrm{S}}, \mu_{\mathrm{M}}, \mu_{\mathrm{MS}}$ for the metric elements between $\langle\mathrm{S}, \mathrm{S}\rangle,\langle\mathrm{M}, \mathrm{M}\rangle$, and $\langle\mathrm{S}, \mathrm{M}\rangle$, respectively.

The appropriate generators for the contracted algebras are obtained after applying the transformations

$$
\begin{aligned}
& g_{\Lambda, \tau, c_{i}} \mathrm{~J}=\mathrm{J}-\beta c_{i}^{2} \mathrm{~S} \quad g_{\Lambda, \tau, c_{i}} \mathrm{~B}_{a}=\left(\tau c_{i}\right)^{-1} \mathrm{~B}_{a} \quad g_{\Lambda, \tau, c_{i}} \mathrm{P}_{a}=\left(\Lambda c_{i}\right)^{-1} \mathrm{P}_{a} \\
& g_{\Lambda, \tau, c_{i}} \mathrm{H}=\left(\Lambda \tau c_{i}^{2}\right)^{-1}\left(c_{i}^{2} \mathrm{H}-\alpha \mathrm{M}\right) \quad g_{\Lambda, \tau, c_{i}} \mathrm{~S}=c_{i}^{-2} \mathrm{~S} \quad g_{\Lambda, \tau, c_{i}} \mathrm{M}=\left(\Lambda \tau c_{i}^{2}\right)^{-1} \mathrm{M},
\end{aligned}
$$

while the new metric elements are given by

$$
g_{\Lambda, \tau, c_{i}} \mu=\mu+\alpha \beta \mu_{\mathrm{MS}} \quad g_{\Lambda, \tau, c_{i}} \chi=\chi+\beta^{2} \mu_{\mathrm{S}}
$$

and

$$
g_{\Lambda, \tau, c_{i}} \mu_{\mathrm{M}}=\left(c_{i} \tau \Lambda\right)^{-2} \mu_{\mathrm{M}} \quad g_{\Lambda, \tau, c_{i}} \mu_{\mathrm{S}}=c_{i}^{-2} \mu_{\mathrm{S}} \quad g_{\Lambda, \tau, c_{i}} \mu_{\mathrm{MS}}=\left(\tau \Lambda c_{i}^{2}\right)^{-1} \mu_{\mathrm{MS}},
$$

which leads us to table 2 . 


\section{A.3 Riemannian spacetimes}

While our analysis was focused on the lorentzian case, it also applies to riemannian spacetimes. One may reintroduce the sign of the metric $\eta_{00}$ (see (A.1)) by substituting $\tau^{2} \rightarrow-\eta_{00} \tau^{2}$ in table 4 . With this change one obtains for $\eta_{00}=+1$ the sphere $\left(\eta_{\text {如 }}=+1\right)$ and hyperbolic space $\left(\eta_{\text {如 }}=-1\right)$, which both lead to euclidean space in the $\Lambda \rightarrow 0$ limit. The analysis in this work generalizes for the three spaces and mirrors the results of their lorentzian counterparts. Otherwise, and connected to the fact that the remaining spacetimes are not lorentzian, the sign $\eta_{00}$ is irrelevant and does not lead to new spacetimes that have not yet been discussed. For the riemannian spaces the "boosts" are compact, which makes their interpretation as a proper spacetime questionable [38].

\section{B Kinematical algebras, homogeneous spaces, and Cartan geometries}

In this appendix we collect relevant background on kinematical algebras and their relation to homogeneous spacetimes. Furthermore, introducing the framework of Cartan geometries clarifies the geometric idea behind the formulation of gravity as a gauge theory. Consult [38, 40, 93] for information on kinematical algebras and homogeneous spacetimes and [94, 95] for background (and hamsters) on Cartan geometries.

Homogeneous spaces. The idea of characterizing geometries by their symmetry groups goes back to Felix Klein and his Erlangen program. Consider a manifold $\mathcal{M}$ and a Lie group $\mathcal{K}$ acting transitively on it. ${ }^{18}$ Assume further that there exist "geometrical structures" on $\mathcal{M}$ that are left invariant by a subgroup $\widetilde{\mathcal{B}}$ of $\mathcal{K}$. Then one can regard the quotient $\mathcal{K} / \widetilde{\mathcal{B}}$ as "the space of geometric structures of type $\widetilde{\mathcal{B}}$ on $\mathcal{M}$ " (see, e.g., [95]). In particular, assume the geometrical structures of interest are the points of the manifold and the subgroup $\mathcal{B}_{x}$ of $\mathcal{K}$ leaves a point $x$ invariant. The quotient $\mathcal{K} / \mathcal{B}_{x}$ is then $\mathcal{K}$-equivariantly diffeomorphic to $\mathcal{M}$. Since a change of base point changes $\mathcal{B}_{x}$ by a conjugation with an element of $\mathcal{K}$, the choice of base point is essentially arbitrary. The manifold $\mathcal{M}$ is therefore equivalently described in terms of the pair $(\mathcal{K}, \mathcal{B})$. The space $\mathcal{K} / \mathcal{B} \simeq \mathcal{M}$ is called Klein geometry or homogeneous space.

Disregarding global properties, homogeneous spaces can be characterized as well by a pair of two Lie algebras $(\mathfrak{k}, \mathfrak{b})$, called Klein pair where $\mathfrak{b}$ is a subalgebra of $\mathfrak{k}$. The dimension of the homogeneous space is given by the difference in dimensions of $\mathfrak{k}$ and $\mathfrak{b}$.

A Klein geometry is called effective if $g x=x$ for all $x \in \mathcal{M}$ implies $g=e$. In other words, the normal subgroup $\mathcal{N}$ of $\mathcal{K}$ must be the identity. ${ }^{19}$ Equivalently, the Lie algebra $\mathfrak{k}$ must not have a non-zero ideal. Any non-effective Klein geometry can be made effective by replacing $(\mathcal{K} / \mathcal{B})$ with $((\mathcal{K} / \mathcal{N}) /(\mathcal{B} / \mathcal{N}))$ where $\mathcal{N}$ is the largest normal subgroup of $\mathcal{B}$.

Kinematical spacetimes. Not every Klein pair will lead to a Klein geometry that has a sensible interpretation as a possible stage for physical theories, i.e., as a kinematical spacetime [38].

\footnotetext{
${ }^{18}$ Let $\mathcal{K} \times \mathcal{M} \rightarrow \mathcal{M}:(g, x) \mapsto g x$ be the action of a group on a manifold. The action is called transitive if for any points $x, y \in \mathcal{M}$ there exists an element $g \in \mathcal{K}$ such that $g x=y$.

${ }^{19}$ A Klein geometry is called locally effective if $\mathcal{N}$ is a discrete group.
} 
In order to have this interpretation it appears reasonable to demand that the spacetime be smooth, connected and isotropic. The latter property implies that the group $\mathcal{K}$ on which the homogeneous space is based must contain the rotation group as a subgroup.

We employ the following definition [40]: a kinematical Lie algebra (with D-dimensional space isotropy) is a real Lie algebra $\mathfrak{k}$ with the following two properties:

- $\mathfrak{k}$ contains a Lie subalgebra $\mathfrak{r} \simeq \mathfrak{s o}(D)$, the Lie algebra of rotations of $D$-dimensional Euclidean space;

- $\mathfrak{k}$ decomposes as $\mathfrak{k}=\mathfrak{r} \oplus 2 V \oplus S$ as a representation of $\mathfrak{r}$, where $2 V$ are two copies of the $D$-dimensional vector irreducible representation of $\mathfrak{s o}(D)$ and $S$ is the onedimensional scalar representation of $\mathfrak{s o}(D)$.

A kinematical Lie group is a Lie group whose Lie algebra is a kinematical Lie algebra.

The decomposition of $\mathfrak{k}$ corresponds to the fact that boosts and spatial translations transform as vectors while time translations transform as scalars. Notice, however, that the identification of the generators in $\mathfrak{k}$ with boosts or spatial momenta cannot be made from the abstract Lie algebra alone. They acquire this interpretation only in the context of a homogeneous kinematical spacetime on which $\mathcal{K}$ acts. We quote the definition of [40]:

A homogeneous kinematical spacetime is a homogeneous space $\mathcal{M}$ of a kinematical Lie group $\mathcal{K}$, satisfying the following properties:

- $\mathcal{M}$ is a connected smooth manifold;

- $\mathcal{K}$ acts transitively and locally effectively on $\mathcal{M}$ with stabilizer $\mathcal{B}$;

- $\mathcal{B}$ is a closed subgroup of $\mathcal{K}$ whose Lie algebra $\mathfrak{b}$ contains a rotational subalgebra $\mathfrak{s o}(D)$ and decomposes as $\mathfrak{b}=\mathfrak{r} \oplus V$ as an adjoint $\mathfrak{r}-$ module, where $V$ is an irreducible $D$-dimensional vector representation of $\mathfrak{s o}(D)$.

The kinematical spacetime is then given by $\mathcal{K} / \mathcal{B}$ and has the dimension $\mathrm{D}+1$. Its properties are equivalently defined by the kinematic Lie pair $(\mathfrak{k}, \mathfrak{b})$.

A classic example of a homogeneous spacetime is $(D+1)$-dimensional Minkowski space. The Poincaré group $\operatorname{ISO}(D, 1)$ acts transitively on this space. Points are left invariant by the Lorentz group $\mathrm{SO}(D, 1)$. Thus, Minkowski space can be described as the homogeneous space $\operatorname{ISO}(D, 1) / \mathrm{SO}(D, 1)$. Working on the infinitesimal level, Minkowski space is characterized by the pair $(\mathfrak{i s o}(D, 1), \mathfrak{s o}(D, 1))$. The Poincaré algebra can be read off from the $\Lambda \rightarrow 0$ limit of table 4 . The Lorentz algebra $\mathfrak{s o}(D, 1)$ is generated by the set $\left\{\mathrm{B}_{a}, \mathrm{~J}_{a b}\right\}$ which are consequently interpreted as boosts and rotations, respectively.

The subalgebra $\mathfrak{b}$ in the kinematic Lie pair thus determines which generators of $\mathfrak{k}$ are to be interpreted as boosts and rotations when acting on the homogeneous spacetime. The remaining generators in $\mathfrak{k} / \mathfrak{b}$ are then identified with spatial translations and time translations. In this work we always assume the following basis for the kinematical algebra $\mathfrak{k}$ :

$$
\mathfrak{k}=\left\{\mathrm{J}_{a b}, \mathrm{~B}_{a}, \mathrm{P}_{a}, \mathrm{H}\right\} .
$$


In order to clarify the importance of the choice of subalgebra $\mathfrak{b}$ in the kinematical Lie pair, take again the Poincaré algebra $\mathfrak{k}=\mathfrak{i s o}(D, 1)$ but quotient now by the subalgebra generated by $\left\{\mathrm{J}_{a b}, \mathrm{P}_{a}\right\}$, that is not isomorphic to the Lorentz algebra. Consequently, the resulting homogeneous spacetime is not Minkowski but the AdS-carrollian spacetime, where now the $\mathrm{P}_{a}$ are interpreted as boosts and the $\mathrm{B}_{a}$ act as spatial translations on the spacetime.

In order to make explicit the choice of subalgebra $\mathfrak{b}$, the basis (B.1) is assumed to be transformed such that $\mathfrak{b}=\left\{\mathrm{J}_{a b}, \mathrm{~B}_{a}\right\}$, i.e., the generators $\mathrm{B}_{a}$ always denote boosts.

Depending on the form of the kinematical Lie pair a homogeneous spacetimes may admit additional geometrical structure invariant under the subgroup $\mathcal{B}$. Accordingly, homogeneous spacetimes can be classified by these invariant structures. For instance, if the homogeneous spacetime $\mathcal{M}$ admits an invariant metric on the tangent space ${ }^{20}$ it is called lorentzian or riemannian depending on the signature of the metric. If a spacetime admits an invariant one-form and "spatial" co-metric, it is called galilean; if it admits an invariant vector and "spatial" metric it is called carrollian. The qualifier "spatial" means that the (co-)metric is degenerate along the invariant (vector) one-form. For even more details concerning the invariants see section 6.1. in [40].

Additional geometric properties of a homogeneous spacetime can be derived from an invariant connection and its curvature and torsion. A sufficient criterion for the existence of an invariant connection is reductiveness of the Lie pair. This means that there exists a split of $(\mathfrak{k}, \mathfrak{b})$ as

$$
\mathfrak{k}=\mathfrak{m} \oplus \mathfrak{b}
$$

such that both parts transform as adjoint $\mathcal{B}$-modules. This means that the action of rotations and boosts does not turn translations into boosts, and vice versa. Notice that the carrollian light cone algebra discussed in section 5.2 is not reductive and does not have an invariant connection. For further details we refer to [40, 93].

Cartan geometries. In the last section we introduced the notion of Klein geometries and homogeneous spacetimes based on the kinematical Lie pair $(\mathfrak{k}, \mathfrak{b})$. The $\mathfrak{k}$-valued field $A$ appearing in the Chern-Simons action, or any other "gauge theory of gravity" for that matter, can be qualitatively understood as "gauging" of this underlying Lie pair. This notion is made precise by the concept of Cartan geometry. Roughly speaking, a Cartan geometry modeled on the homogeneous space $\mathcal{K} / \mathcal{B}$ is a manifold $\mathcal{M}$ on which the linear tangent space is replaced by the homogeneous space $\mathcal{K} / \mathcal{B}$ at every point. These geometries are therefore generalizations of riemannian geometries, as summarized in the following diagram (from $[94,95]$ ).

We will refrain from giving the precise definition of a Cartan geometry modeled on $\mathcal{K} / \mathcal{B}$ which is most naturally phrased as a principal $\mathcal{B}$-bundle $P \rightarrow \mathcal{M}$ with a $\mathfrak{k}$-valued 1 -form on $P$, the Cartan connection. It is precisely this connection that becomes the dynamical

\footnotetext{
${ }^{20}$ This is not to be confused with an invariant metric on the kinematical Lie algebra of the homogeneous spacetimes.
} 


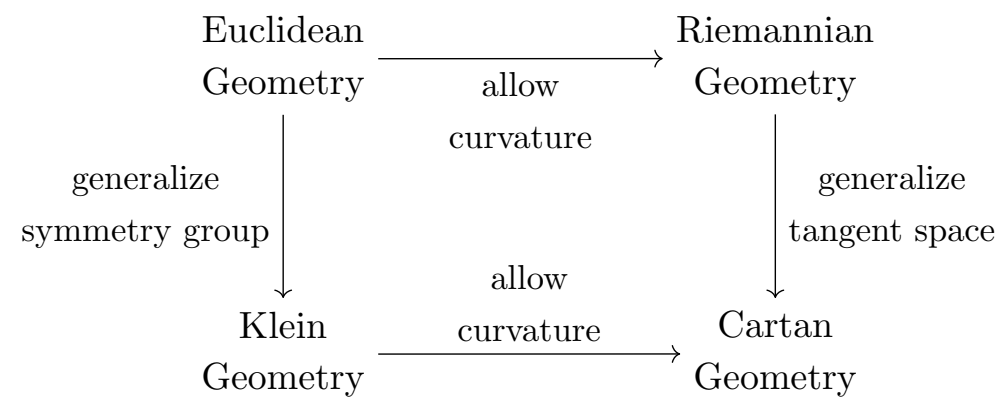

Figure 2. Cartan geometry is a generalization of both Riemannian geometry, by allowing the tangent spaces to be different from $\mathbb{R}^{n}$, and of Klein geometry, by nontrivially gluing together Klein geometries.

quantity in a gauge theory of gravity. In particular, the Chern-Simons connection (2.2) is recognized as a Cartan connection $A .{ }^{21}$

The curvature $F$ of the Cartan connection, which is a $\mathfrak{k}$-valued two-form, determines the amount by which the Cartan geometry deviates from the homogeneous space on which it is modeled. In particular, the curvature vanishes if the Cartan geometry itself is a homogeneous space (or a quotient thereof). Even if the Cartan geometry is flat, i.e., the curvature vanishes, the homogeneous space on which the geometry is modeled can have curvature. The curvature of the homogeneous space is given by projecting $F$ to $\mathfrak{b}$, whereas the projection of $F$ to $\mathfrak{k} / \mathfrak{b}$ calculates the torsion of the geometry. Both of these statements are easily checked using the classic example of $\mathrm{AdS}_{3}$ gravity.

\section{The full action and equations of motion}

While the following formulas might seem intricate let us emphasize that the presentation is rather economic since we are describing the theories of figure 1 all at once. The most general action (3.16) can be fully decomposed to take the following form

$$
\begin{aligned}
S=\int[2 \hat{R} \wedge h & +2\left(c_{i}^{2} \mu+\alpha \beta \mu_{\mathrm{MS}}\right) \hat{R}_{a} \wedge p_{a}+\hat{\Lambda}+L(\omega) \\
+ & \left.\hat{T} \wedge h \pm \Lambda^{2}\left(c_{i}^{2} \chi+\beta^{2} \mu_{\mathrm{S}}\right) \hat{T}_{a} \wedge p_{a}+c_{i}^{2} L_{M, S}+L_{I}\right]
\end{aligned}
$$

where we have dropped various boundary terms and use

$$
\begin{aligned}
\hat{R} & =-\mu d j+\frac{1}{2}\left(c_{i}^{2} \mu+\alpha \beta \mu_{\mathrm{MS}}\right) \tau^{2} \epsilon_{a b} b_{a} \wedge b_{b} \\
\hat{R}_{a} & =d b_{a}+\epsilon_{a b} b_{b} \wedge j
\end{aligned}
$$

\footnotetext{
${ }^{21}$ Note that this is a crucial difference to the concept of an Ehresmann connection on a principal $\mathcal{B}$-bundle over $\mathcal{M}$ which would be given by a $\mathfrak{b}$-valued one-form. Ehresmann connections are the dynamical variables in usual gauge theories as, e.g., Yang-Mills. Thus, gauge theories of gravity are a priori different from gauge theories of Yang-Mills type. Nevertheless, given some requirements the principal bundles with Ehresmann connections and Cartan connections can be shown to be equivalent [94].
} 


$$
\begin{aligned}
\hat{\Lambda} & = \pm \Lambda^{2}\left(c_{i}^{2} \mu+\alpha \beta \mu_{\mathrm{MS}}\right) \epsilon_{a b} p_{a} \wedge p_{b} \wedge h \\
L(\omega) & =-\chi j \wedge d j+\tau^{2}\left(c_{i}^{2} \chi+\beta^{2} \mu_{\mathrm{S}}\right)\left(b_{a} \wedge d b_{a}+\epsilon_{a b} b_{a} \wedge b_{b} \wedge j\right) \\
\hat{T} & =\left[\mp \Lambda^{2} \tau^{2} \chi+\frac{1}{c_{i}^{2}}\left(\alpha^{2} \mu_{\mathrm{M}} \mp \Lambda^{2} \tau^{2} \beta^{2} \mu_{\mathrm{S}}\right)\right] d h \pm \tau^{2} \Lambda^{2}\left(c_{i}^{2} \chi+\beta^{2} \mu_{\mathrm{S}}\right) \epsilon_{a b} b_{a} \wedge p_{b} \\
\hat{T}_{a} & =d p_{a}+\tau^{2} \epsilon_{a b} b_{b} \wedge h+\epsilon_{a b} p_{b} \wedge j \\
L_{M, S} & =\mu_{\mathrm{M}} m \wedge d m+\mu_{\mathrm{S}} s \wedge d s+2 \mu_{\mathrm{MS}} m \wedge d s \\
L_{I} & =2 \alpha\left(\mu_{\mathrm{M}} h \wedge d m+\mu_{\mathrm{MS}} h \wedge d s\right)+2 \beta\left(\mu_{\mathrm{MS}} j \wedge d m+\mu_{\mathrm{S}} j \wedge d s\right) .
\end{aligned}
$$

The equations of motion $F\left(\mathrm{x}_{A}\right) \equiv\left\langle F, \mathrm{x}_{A}\right\rangle$ that follow from the variational principle are then given by

$$
\begin{aligned}
F(\mathrm{H})= & -2 \mu d h+2\left(c_{i}^{2} \mu+\alpha \beta \mu_{\mathrm{MS}}\right) \epsilon_{a b} b_{a} \wedge p_{b}-2 \chi d j+\tau^{2}\left(c_{i}^{2} \chi+\beta^{2} \mu_{\mathrm{S}} \epsilon_{a b} b_{a} \wedge b_{b}\right) \\
& \pm \Lambda^{2}\left(c_{i}^{2} \chi+\beta^{2} \mu_{\mathrm{S}}\right) \epsilon_{a b} p_{a} \wedge p_{b}+2 \beta\left(\mu_{\mathrm{MS}} d m+\mu_{\mathrm{S}} d s\right)=0 \\
F\left(\mathrm{P}_{a}\right)= & \left(c_{i}^{2} \mu+\alpha \beta \mu_{\mathrm{MS}}\right) \hat{T}_{a}+\tau^{2}\left(c_{i}^{2} \chi+\beta^{2} \mu_{\mathrm{S}}\right)\left(\hat{R}_{a} \pm \Lambda^{2} \epsilon_{a b} p_{b} \wedge h\right)=0 \\
F(\mathrm{~J})= & \hat{R} \pm \Lambda^{2}\left(c_{i}^{2} \mu+\alpha \beta \mu_{\mathrm{MS}}\right) \epsilon_{a b} p_{a} \wedge p_{b}+2 \hat{T}+2 \alpha\left(\mu_{\mathrm{M}} d m+\mu_{\mathrm{MS}} d s\right)=0 \\
F\left(\mathrm{~B}_{a}\right)= & \pm \Lambda^{2}\left(c_{i}^{2} \chi+\beta^{2} \mu_{\mathrm{S}}\right) \hat{T}_{a}+\left(c_{i}^{2} \mu+\alpha \beta \mu_{\mathrm{MS}}\right)\left(\hat{R}_{a} \pm \Lambda^{2} \epsilon_{a b} p_{b} \wedge h\right)=0 \\
F(\mathrm{~S})= & c_{i}^{2}\left(\mu_{\mathrm{S}} d s+\mu_{\mathrm{MS}} d m\right)+\alpha \mu_{\mathrm{MS}} d h+\beta \mu_{\mathrm{S}} d j=0 \\
F(\mathrm{M})= & c_{i}^{2}\left(\mu_{\mathrm{M}} d m+\mu_{\mathrm{MS}} d s\right)+\alpha \mu_{\mathrm{M}} d h+\beta \mu_{\mathrm{MS}} d j=0
\end{aligned}
$$

Under an infinitesimal gauge transformation $\delta A=d \varepsilon+[A, \varepsilon]$ spanned by a parameter $\varepsilon=\omega \mathrm{H}+\lambda^{a} \mathrm{P}_{a}+\xi \mathrm{J}+\sigma^{a} \mathrm{~B}_{a}+\omega^{*} \mathrm{~S}+\xi^{*} \mathrm{M}$ valued in the algebra in table 2 , the components of the gauge field transform according to

$$
\begin{aligned}
\delta h & =d \omega+c_{i}^{2} \epsilon_{a b}\left(-b_{a} \lambda_{b}+p_{a} \sigma_{b}\right) \\
\delta p_{a} & =d \lambda_{a}-\epsilon_{a b} j \lambda_{b}+\epsilon_{a b} p_{b} \xi+\tau^{2} \epsilon_{a b}\left(b_{b} \omega-h \sigma_{b}\right) \\
\delta j & =d \xi-c_{i}^{2} \epsilon_{a b}\left(\tau^{2} b_{a} \sigma_{b} \pm \Lambda^{2} p_{a} \lambda_{b}\right) \\
\delta b_{a} & =d \sigma_{a}-\epsilon_{a b} j \sigma_{b}+\epsilon_{a b} b_{b} \xi \pm \Lambda^{2} \epsilon_{a b}\left(p_{b} \omega-h \lambda_{b}\right) \\
\delta m & =d \xi^{*}+\alpha \epsilon_{a b}\left(b_{a} \lambda_{b}-p_{a} \sigma_{b}\right) \\
\delta s & =d \omega^{*}+\beta \epsilon_{a b}\left(\tau^{2} b_{a} \sigma_{b} \pm \Lambda^{2} p_{a} \lambda_{b}\right) .
\end{aligned}
$$

Open Access. This article is distributed under the terms of the Creative Commons Attribution License (CC-BY 4.0), which permits any use, distribution and reproduction in any medium, provided the original author(s) and source are credited.

\section{References}

[1] A. Achucarro and P.K. Townsend, A Chern-Simons action for three-dimensional Anti-de Sitter supergravity theories, Phys. Lett. B 180 (1986) 89 [InSPIRE]. 
[2] E. Witten, $(2+1)$-dimensional gravity as an exactly soluble system, Nucl. Phys. B 311 (1988) 46 [INSPIRE].

[3] J.D. Brown and M. Henneaux, Central charges in the canonical realization of asymptotic symmetries: an example from three-dimensional gravity, Commun. Math. Phys. 104 (1986) 207 [INSPIRE].

[4] J.M. Maldacena, The large $N$ limit of superconformal field theories and supergravity, Int. J. Theor. Phys. 38 (1999) 1113 [Adv. Theor. Math. Phys. 2 (1998) 231] [hep-th/9711200] [INSPIRE].

[5] S.S. Gubser, I.R. Klebanov and A.M. Polyakov, Gauge theory correlators from noncritical string theory, Phys. Lett. B 428 (1998) 105 [hep-th/9802109] [INSPIRE].

[6] E. Witten, Anti-de Sitter space and holography, Adv. Theor. Math. Phys. 2 (1998) 253 [hep-th/9802150] [INSPIRE].

[7] E. Witten, Quantum field theory and the Jones polynomial, Commun. Math. Phys. 121 (1989) 351 [INSPIRE].

[8] S. Elitzur, G.W. Moore, A. Schwimmer and N. Seiberg, Remarks on the canonical quantization of the Chern-Simons-Witten theory, Nucl. Phys. B 326 (1989) 108 [INSPIRE].

[9] O. Coussaert, M. Henneaux and P. van Driel, The asymptotic dynamics of three-dimensional Einstein gravity with a negative cosmological constant, Class. Quant. Grav. 12 (1995) 2961 [gr-qc/9506019] [INSPIRE].

[10] D.T. Son, Toward an AdS/cold atoms correspondence: a geometric realization of the Schrödinger symmetry, Phys. Rev. D 78 (2008) 046003 [arXiv:0804.3972] [INSPIRE].

[11] K. Balasubramanian and J. McGreevy, Gravity duals for non-relativistic CFTs, Phys. Rev. Lett. 101 (2008) 061601 [arXiv:0804.4053] [INSPIRE].

[12] S. Kachru, X. Liu and M. Mulligan, Gravity duals of Lifshitz-like fixed points, Phys. Rev. D 78 (2008) 106005 [arXiv: 0808.1725] [INSPIRE].

[13] A. Bagchi and R. Gopakumar, Galilean conformal algebras and AdS/CFT, JHEP 07 (2009) 037 [arXiv:0902.1385] [INSPIRE].

[14] A. Bagchi, R. Gopakumar, I. Mandal and A. Miwa, GCA in 2d, JHEP 08 (2010) 004 [arXiv:0912.1090] [INSPIRE].

[15] M.H. Christensen, J. Hartong, N.A. Obers and B. Rollier, Torsional Newton-Cartan geometry and Lifshitz holography, Phys. Rev. D 89 (2014) 061901 [arXiv:1311.4794] [INSPIRE].

[16] M.H. Christensen, J. Hartong, N.A. Obers and B. Rollier, Boundary stress-energy tensor and Newton-Cartan geometry in Lifshitz holography, JHEP 01 (2014) 057 [arXiv:1311.6471] [INSPIRE].

[17] M. Taylor, Lifshitz holography, Class. Quant. Grav. 33 (2016) 033001 [arXiv:1512.03554] [INSPIRE].

[18] D.T. Son, Newton-Cartan geometry and the quantum Hall effect, arXiv: 1306.0638 [INSPIRE].

[19] C. Hoyos and D.T. Son, Hall viscosity and electromagnetic response, Phys. Rev. Lett. 108 (2012) 066805 [arXiv:1109.2651] [INSPIRE].

[20] A. Gromov, K. Jensen and A.G. Abanov, Boundary effective action for quantum Hall states, Phys. Rev. Lett. 116 (2016) 126802 [arXiv:1506.07171] [INSPIRE]. 
[21] M. Geracie, K. Prabhu and M.M. Roberts, Curved non-relativistic spacetimes, Newtonian gravitation and massive matter, J. Math. Phys. 56 (2015) 103505 [arXiv:1503.02682] [INSPIRE].

[22] M. Blau and M. O'Loughlin, Horizon shells and BMS-like soldering transformations, JHEP 03 (2016) 029 [arXiv: 1512.02858] [INSPIRE].

[23] R.F. Penna, Near-horizon Carroll symmetry and black hole Love numbers, arXiv: 1812.05643 [INSPIRE].

[24] C. Troessaert, The BMS4 algebra at spatial infinity, Class. Quant. Grav. 35 (2018) 074003 [arXiv: 1704.06223] [INSPIRE].

[25] M. Henneaux and C. Troessaert, BMS group at spatial infinity: the Hamiltonian (ADM) approach, JHEP 03 (2018) 147 [arXiv: 1801.03718] [INSPIRE].

[26] G.W. Gibbons, The Ashtekar-Hansen universal structure at spatial infinity is weakly pseudo-Carrollian, arXiv: 1902.09170 [INSPIRE].

[27] H. Bondi et al., Gravitational waves in general relativity. 7. Waves from axisymmetric isolated systems, Proc. Roy. Soc. Lond. A 269 (1962) 21.

[28] R. Sachs, Asymptotic symmetries in gravitational theory, Phys. Rev. 128 (1962) 2851 [INSPIRE].

[29] C. Duval, G.W. Gibbons and P.A. Horvathy, Conformal Carroll groups and BMS symmetry, Class. Quant. Grav. 31 (2014) 092001 [arXiv:1402 .5894] [INSPIRE].

[30] J. Hartong, Gauging the Carroll algebra and ultra-relativistic gravity, JHEP 08 (2015) 069 [arXiv: 1505.05011] [INSPIRE].

[31] E. Bergshoeff, D. Grumiller, S. Prohazka and J. Rosseel, Three-dimensional spin-3 theories based on general kinematical algebras, JHEP 01 (2017) 114 [arXiv:1612.02277] [INSPIRE].

[32] E. Bergshoeff et al., Carroll versus Galilei gravity, JHEP 03 (2017) 165 [arXiv:1701.06156] [INSPIRE].

[33] G. Papageorgiou and B.J. Schroers, A Chern-Simons approach to galilean quantum gravity in $2+1$ dimensions, JHEP 11 (2009) 009 [arXiv:0907.2880] [INSPIRE].

[34] G. Papageorgiou and B.J. Schroers, Galilean quantum gravity with cosmological constant and the extended q-Heisenberg algebra, JHEP 11 (2010) 020 [arXiv:1008.0279] [INSPIRE].

[35] E.A. Bergshoeff and J. Rosseel, Three-dimensional extended Bargmann supergravity, Phys. Rev. Lett. 116 (2016) 251601 [arXiv:1604.08042] [INSPIRE].

[36] J. Hartong, Y. Lei and N.A. Obers, Nonrelativistic Chern-Simons theories and three-dimensional Hořava-Lifshitz gravity, Phys. Rev. D 94 (2016) 065027 [arXiv: 1604.08054] [INSPIRE].

[37] E. Joung and W. Li, Nonrelativistic limits of colored gravity in three dimensions, Phys. Rev. D 97 (2018) 105020 [arXiv: 1801.10143] [INSPIRE].

[38] H. Bacry and J. Levy-Leblond, Possible kinematics, J. Math. Phys. 9 (1968) 1605 [INSPIRE].

[39] H. Bacry and J. Nuyts, Classification of ten-dimensional kinematical groups with space isotropy, J. Math. Phys. 27 (1986) 2455 [INSPIRE].

[40] J. Figueroa-O'Farrill and S. Prohazka, Spatially isotropic homogeneous spacetimes, JHEP 01 (2019) 229 [arXiv:1809.01224] [INSPIRE]. 
[41] J. Figueroa-O'Farrill, Classification of kinematical Lie algebras, arXiv:1711.05676 [INSPIRE].

[42] A. Medina and P. Revoy, Algèbres de Lie et produit scalaire invariant, Ann. Sci. E.N.S. 18 (1985) 553.

[43] J.M. Figueroa-O'Farrill and S. Stanciu, On the structure of symmetric selfdual Lie algebras, J. Math. Phys. 37 (1996) 4121 [hep-th/9506152] [INSPIRE].

[44] S.N. Solodukhin, Holography with gravitational Chern-Simons, Phys. Rev. D 74 (2006) 024015 [hep-th/0509148] [INSPIRE].

[45] M.-I. Park, BTZ black hole with gravitational Chern-Simons: thermodynamics and statistical entropy, Phys. Rev. D 77 (2008) 026011 [hep-th/0608165] [INSPIRE].

[46] P.K. Townsend and B. Zhang, Thermodynamics of "exotic" Bañados-Teitelboim-Zanelli black holes, Phys. Rev. Lett. 110 (2013) 241302 [arXiv:1302.3874] [INSPIRE].

[47] D.M. Hofman and B. Rollier, Warped conformal field theory as lower spin gravity, Nucl. Phys. B 897 (2015) 1 [arXiv:1411.0672] [INSPIRE].

[48] J. Hartong, Y. Lei, N.A. Obers and G. Oling, Zooming in on $A d S_{3} / C F T_{2}$ near a BPS bound, JHEP 05 (2018) 016 [arXiv: 1712.05794] [INSPIRE].

[49] L. Avilés et al., Non-relativistic Maxwell Chern-Simons gravity, JHEP 05 (2018) 047 [arXiv: 1802.08453] [INSPIRE].

[50] A. Fialowski and M. de Montigny, On deformations and contractions of Lie algebras, SIGMA 2 (2006) 048 [math/0605091].

[51] A. Fialowski and M. de Montigny, Deformations and contractions of Lie algebras, J. Phys. A 38 (2005) 6335.

[52] K. Morand, Embedding Galilean and Carrollian geometries I. Gravitational waves, arXiv: 1811.12681 [INSPIRE].

[53] J.A. de Azcárraga and J.M. Izquierdo, Lie groups, Lie algebras, cohomology and some applications in physics, Cambridge University Press, Cambridge U.K. (2011).

[54] J.M. Levy-Léblond, Galilei group and galilean invariance, in Group theory and its applications, E.M. Loebl ed., Academic Press, U.S.A. (1971).

[55] T. Andrzejewski and J.M. Figueroa-O'Farrill, Kinematical Lie algebras in $2+1$ dimensions, J. Math. Phys. 59 (2018) 061703 [arXiv:1802.04048] [INSPIRE].

[56] S. Kobayashi and K. Nomizu, Foundations of differential geometry. Volume II, Wiley Classics Library, New York U.S.A. (1996).

[57] E.W. Mielke and P. Baekler, Topological gauge model of gravity with torsion, Phys. Lett. A 156 (1991) 399 [INSPIRE].

[58] P. Baekler, E.W. Mielke and F.W. Hehl, Dynamical symmetries in topological 3D gravity with torsion, Nuovo Cim. B 107 (1992) 91 [INSPIRE].

[59] M. Blagojevic and M. Vasilic, 3D gravity with torsion as a Chern-Simons gauge theory, Phys. Rev. D 68 (2003) 104023 [gr-qc/0307078] [INSPIRE].

[60] S.L. Cacciatori et al., Chern-Simons formulation of three-dimensional gravity with torsion and nonmetricity, J. Geom. Phys. 56 (2006) 2523 [hep-th/0507200] [INSPIRE]. 
[61] A. Giacomini, R. Troncoso and S. Willison, Three-dimensional supergravity reloaded, Class. Quant. Grav. 24 (2007) 2845 [hep-th/0610077] [INSPIRE].

[62] A.H. Chamseddine, Topological gauge theory of gravity in five-dimensions and all odd dimensions, Phys. Lett. B 233 (1989) 291 [INSPIRE].

[63] A.H. Chamseddine, Topological gravity and supergravity in various dimensions, Nucl. Phys. B 346 (1990) 213 [INSPIRE].

[64] M. Bañados, R. Troncoso and J. Zanelli, Higher dimensional Chern-Simons supergravity, Phys. Rev. D 54 (1996) 2605 [gr-qc/9601003] [InSPIRE].

[65] S. Gryb and F. Mercati, $2+1$ gravity on the conformal sphere, Phys. Rev. D 87 (2013) 064006 [arXiv: 1209.4858 ] [inSPIRE].

[66] S. Bonanos and J. Gomis, A note on the Chevalley-Eilenberg cohomology for the Galilei and Poincaré algebras, J. Phys. A 42 (2009) 145206 [arXiv:0808.2243] [inSPIRE].

[67] R. Schrader, The Maxwell group and the quantum theory of particles in classical homogeneous electromagnetic fields, Fortsch. Phys. 20 (1972) 701 [INSPIRE].

[68] H. Bacry, P. Combe and J.L. Richard, Group-theoretical analysis of elementary particles in an external electromagnetic field. 1. The relativistic particle in a constant and uniform field, Nuovo Cim. A 67 (1970) 267 [INSPIRE].

[69] D.V. Soroka and V.A. Soroka, Tensor extension of the Poincaré' algebra, Phys. Lett. B 607 (2005) 302 [hep-th/0410012] [INSPIRE].

[70] J.M. Figueroa-O'Farrill, Higher-dimensional kinematical Lie algebras via deformation theory, J. Math. Phys. 59 (2018) 061702 [arXiv:1711.07363] [INSPIRE].

[71] S. Prohazka, Chern-Simons holography: boundary conditions, contractions and double extensions for a journey beyond Anti-de Sitter, Ph.D. thesis, Technische Universität Wien, Vienna, Austria (2017), arXiv:1710.11110 [INSPIRE].

[72] S. Giombi, A. Maloney and X. Yin, One-loop partition functions of $3 D$ gravity, JHEP 08 (2008) 007 [arXiv: 0804.1773] [INSPIRE].

[73] J.R. David, M.R. Gaberdiel and R. Gopakumar, The heat kernel on $A d S_{3}$ and its applications, JHEP 04 (2010) 125 [arXiv:0911.5085] [INSPIRE].

[74] F. Denef, S.A. Hartnoll and S. Sachdev, Black hole determinants and quasinormal modes, Class. Quant. Grav. 27 (2010) 125001 [arXiv:0908.2657] [INSPIRE].

[75] A. Castro, C. Keeler and P. Szepietowski, Tweaking one-loop determinants in AdS $S_{3}$, JHEP 10 (2017) 070 [arXiv:1707.06245] [INSPIRE].

[76] M. Porrati and C. Yu, Kac-Moody and Virasoro characters from the perturbative Chern-Simons path integral, JHEP 05 (2019) 083 [arXiv: 1903.05100] [INSPIRE].

[77] G. Barnich, H.A. Gonzalez, A. Maloney and B. Oblak, One-loop partition function of three-dimensional flat gravity, JHEP 04 (2015) 178 [arXiv:1502.06185] [INSPIRE].

[78] M. Ammon, A. Castro and N. Iqbal, Wilson lines and entanglement entropy in higher spin gravity, JHEP 10 (2013) 110 [arXiv: 1306.4338] [INSPIRE].

[79] J. de Boer and J.I. Jottar, Entanglement entropy and higher spin holography in AdS $S_{3}, J H E P$ 04 (2014) 089 [arXiv: 1306.4347] [INSPIRE]. 
[80] D. Grumiller, W. Merbis and M. Riegler, Most general flat space boundary conditions in three-dimensional Einstein gravity, Class. Quant. Grav. 34 (2017) 184001 [arXiv: 1704.07419] [INSPIRE].

[81] D. Grumiller and M. Riegler, Most general AdS 3 boundary conditions, JHEP 10 (2016) 023 [arXiv: 1608.01308] [INSPIRE].

[82] N. Mohammedi, On bosonic and supersymmetric current algebras for nonsemisimple groups, Phys. Lett. B 325 (1994) 371 [hep-th/9312182] [INSPIRE].

[83] J.M. Figueroa-O'Farrill and S. Stanciu, Nonsemisimple Sugawara constructions, Phys. Lett. B 327 (1994) 40 [hep-th/9402035] [INSPIRE].

[84] M. Henneaux, Geometry of zero signature space-times, Bull. Soc. Math. Belg. 31 (1979) 47 [INSPIRE].

[85] A. Campoleoni et al., Two-dimensional fluids and their holographic duals, arXiv: 1812.04019 [INSPIRE].

[86] P. Concha, N. Merino et al., Asymptotic symmetries of three-dimensional Chern-Simons gravity for the Maxwell algebra, JHEP 10 (2018) 079 [arXiv: 1805.08834] [INSPIRE].

[87] T.G. Mertens, The Schwarzian theory - Origins, JHEP 05 (2018) 036 [arXiv:1801.09605] [INSPIRE].

[88] H.A. González, D. Grumiller and J. Salzer, Towards a bulk description of higher spin SYK, JHEP 05 (2018) 083 [arXiv: 1802.01562] [INSPIRE].

[89] J. Maldacena and D. Stanford, Remarks on the Sachdev-Ye-Kitaev model, Phys. Rev. D 94 (2016) 106002 [arXiv:1604.07818] [INSPIRE].

[90] D. Hansen, J. Hartong and N.A. Obers, Action principle for newtonian gravity, Phys. Rev. Lett. 122 (2019) 061106 [arXiv: 1807.04765] [INSPIRE].

[91] E.A. Bergshoeff, K.T. Grosvenor, C. Simsek and Z. Yan, An action for extended string Newton-Cartan gravity, JHEP 01 (2019) 178 [arXiv: 1810.09387] [INSPIRE].

[92] M. Le Bellac and J.M. Lévy-Leblond, Galilean electromagnetism, Nuovo Cim. B 14 (1973) 217.

[93] J. Figueroa-O'Farrill, R. Grassie and S. Prohazka, Geometry and BMS Lie algebras of spatially isotropic homogeneous spacetimes, arXiv:1905.00034 [INSPIRE].

[94] R. Sharpe and S. Chern, Differential geometry: Cartan's generalization of Klein's Erlangen program, Graduate Texts in Mathematics, Springer, Germany (2000).

[95] D.K. Wise, MacDowell-Mansouri gravity and Cartan geometry, Class. Quant. Grav. 27 (2010) 155010 [gr-qc/0611154] [INSPIRE]. 